580
$5 a 25$

Nomenclature of

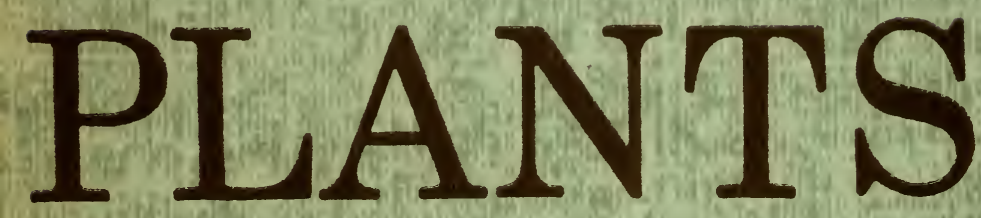

A text for the Application by the Case Method of the International Code of Botanical Nomenclature

Harold St. John

THE RONALD PRESS COMPANY · NEW YORK 


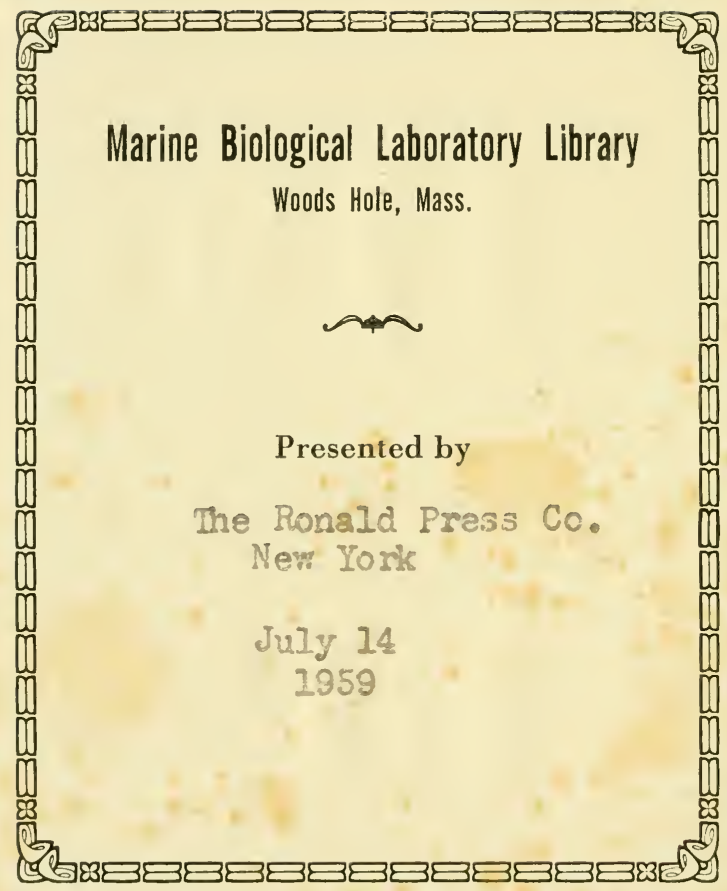




\title{
CHRONICA BOTANICA
}

NEW. SERIES OF PLANT SCIENCE BOOKS

\author{
Consulting Editor, Frans Verdoorn
}

No. 31 



\section{NOMENCLATURE OF PLANTS}

A Text for the Application by the

Case Method

of the International Code of

Botanical Nomenclature

\section{HAROLD ST. JOHN}

G. P. WILDER PROFESSOR OF BOTANY

UNIVERSITY OF HAWAII, AND

BOTANIST, B. P. BISHOP MUSEUM, HONOLULU 
Copyright, (C), 1958, by THE RONALD PRESS COMPANY

\author{
All Rights Reserved
}

The text of this publication or any part thereof may not be reproduced in any manner whatsoever without permission in writing from the publisher. 


\section{PREFACE}

In recent times systematic botany has gained increased recognition, and this is reflected by the ever-larger number of schools, colleges, and universities that include it in their formal program of instruction. The taxonomic part is taught at length by lecture, textbook, laboratory work, and on field excursions.

On the other hand, nomenclature is usually covered briefly in a week or two of lectures. These are mostly historical in viewpoint, as opposed to practical. Such lectures are of value and are interesting, but the student does not gain a detailed knowledge of the content of the laws or the nomenclatural procedure under them as codified in the International Code of Botanical Nomenclature. He does not learn the basic principles of nomenclature. Usually his only real contact with it is by a chance overhearing of an argument between two of his instructors as to which is the correct name of a particular plant. Some taxonomists announce to their students that nomenclature is an evil, though they admit its necessity. They argue that a botanist should spend all his time studying the plants themselves, and that time spent on nomenclature is time wasted.

On the contrary, a taxonomic report published with the taxonomy or classification well done, but the nomenclature incomplete or incorrect, is not authoritative and must be done over again by some competent investigator.

After a short, well-directed study, nomenclature becomes first interesting, then fascinating. Its study reveals much of the development of botany, and it gives a familiarity with the beginnings of the science, the pioneer workers on, and the great books of, our science.

For more than a decade the writer has taught a course on botanical nomenclature at the University of Hawaii. The response by the students has been such as to cause him to expand the 



\title{
CONTENTS
}

\author{
1 \\ Introduction, 1
}

2

Cases 1 Through 188,10

3

Cases 200 Through 299, 37

4

Cases 300 Through 460, 52

5

Cases 500 Through 598, 74

6

Cases 600 Through 688, 88

7

Cases 700 Through 958, 99

Index, 131 



\section{NOMENCLATURE OF PLANTS}

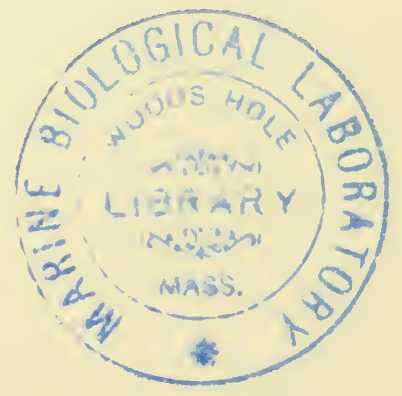





\section{CHAPTER 1}

\section{INTRODUCTION}

Nomenclature is an inseparable and necessary part of the science and art of systematic botany. On a factual basis, such as the distinctive morphology, samples of a plant population are classified as a distinct unit, now called a taxon. On evaluation of its resemblances to, and differences from, other taxa, the botanist decides upon its taxonomy, that is, the placement of the taxon into the general system of classification. When this placement is determined, then the correct scientific name, according to plant nomenclature, must be found. The existing names must be judged as to whether or not they are effectively published, validly published, and with priority. If no available name exists, a new one must be formed. These two steps in the establishment of a taxon are, respectively, taxonomic and nomenclatural. Systematic work is correctly and fully done only when both of these steps are properly carried out.

Though they have had various and even identical definitions in botanical texts and dictionaries, the following botanical terms are used in this text as here defined:

Systematic botany or systematics embraces the whole field of systematic work, including the placing of an individual plant into a category of classification or taxon, the assignment of the taxon into the system of orderly arrangement or phylogeny, and the selection of a scientific name. This is divisible into the two following parts:

Taxonomy deals with the placement of a plant into its smallest taxon, and that taxon into the hierarchy of larger ones in the system. All such modern systems are phylogenetic in character.

Nomenclature deals with the selection of the correct scientific name for a plant when placed in a particular taxon, in conformity with the legal requirements, that is, with the articles and rec- 
ommendations of the last International Code of Botanical Nomenclature.

The new terms neosystematics and biosystematy are not used here, as they seem based on an overemphasis of the fact that in recent times genetics and ecology have provided some new evidence to be included with the morphologic in reaching taxonomic judgements.

A very effective way of training botanical students in nomenclature is by having them take part in the doing of it. This is easily arranged and supervised by assigning the students jointly or singly to solve a series of nomenclatural cases. Each student who is assigned a case investigates it, finds and evaluates each publication cited, searches for other pertinent facts, for other names, such as homonyms, nomina conservanda, etc., then, when ready, reports on the case. The student gives his interpretations before the class, the members of which serve as the jury. Queries and arguments bring out the features of the case. The instructor, serving as judge, guides the discussion, confirms the opinion of the jury, or, if necessary, gives his own judgement. This method of presentation has proved stimulating to the individual students.

There is given below, as an example, the case of Cydonia Cydonia, and the process, step by step, of its investigation and solution. The others can be digested and solved in a similar way, and the study of a series of these cases will aid in giving students a sounder training in botany.

\section{Solution of a Sample Nomenclatural Case}

Cydonia Cydonia (L.) Pers., Syn. Pl. 2: 40, 1807.

Pyrus Cydonia L., Sp. Pl. 480, 1753.

Cydonia Oblonga Mill., Gard. Dict., ed. 8, Cydonia No. 1, 1768. C. vulgaris Pers., Syn. Pl. 2: Corrigenda for p. 40, 1807.

The earliest of these names is Pyrus Cydonia, which is found in Linnaeus' Species Plantamum, 1753. There on page 480 Linnaeus published this name for the quince tree, found on the rocky banks of the Danube. This name appeared in his printed book, which was offered for sale in 1753, hence, it was effectively published, under Art. 29 (1956), which states, "Publica- 
tion is effected, under this Code, only by distribution of printed matter (through sale, exchange, or gift) to the general public or at least to botanical institutions with libraries accessible to botanists generally." The name was validly published, under Art. 13, which provides, "Valid publication ... is treated as beginning,... a. Spermatophyta and Pteridophyta, 1 May 1753 (Linnaeus, Species Plantamum, ed. 1)."

It is published with references to earlier printed descriptions in two of his own books, one book by Royen, and one by Bauhin. This satisfies Art. 32(2), which requires that, "In order to be validly published, a name of a taxon of recent plants must be both (1) effectively published ... and (2) accompanied by a description of the taxon or by a reference (direct or indirect) to a previously and effectively published description of it."

It is validly published with the new specific epithet to be associated as a binomial with the generic name, under Art. 32, fourth paragraph, which reads, "A combination is not validly published unless the author definitely indicates that the epithet or epithets concerned are to be used in that particular combination.... Examples, In Linnaeus' Species Plantarum the placing of the epithet in the margin opposite the name of the genus clearly indicates the combination intended."

Cydonia Oblonga Miller, published in his great Gardeners Dictionary, ed. 8, 1768, was effectively published. It contained a description and the common name, Quince-tree. It also gave a reference to the earlier Cydonia fmuctu oblongo laeviori of Tournefort's Institutiones Rei Herbarii, 632, a book published in 1700. There the genus was described and illustrated, and the name quoted above given for the quince. This was published before the starting date of botanical nomenclature, 1753 (Art. 13), and the species was given not a binomial but a polynomial, which is now illegitimate under Art. 23, which states: "The name of a species is a binary combination consisting of the name of the genus followed by a single specific epithet." Consequently, the name published by Tournefort is illegitimate, but the description and figure given by him can be cited as the basis of a binomial that was published in or after 1753. Miller's Gardeners Dictionary was without numbered pages, instead it was organized like an encyclopaedia, with the 
entries in alphabetical order. Under Cydonia, with three species, it said, "The species are, 1. Cydonia (Oblonga) foliis oblongo-ovatis subtus tomentosis, pomis oblongis basi productis. Quince-tree with oblong oval leaves, woolly on their under side, and an oblong fruit lengthened at the base." Miller discussed the grouping by Linnaeus of the apple, pear, and quince in the same genus, Pyms. He opined that the quince and the pear were nearly allied botanically, but the apple not so. Then, in the section on propagation, he continued, "As the Pear will take upon the Quince by grafting or budding, and so vice versa, we may conclude there is a near alliance between them; but as neither of these will take upon the Apple, nor that upon either of these, so we should separate them under different genera, as will be further mentioned under the article Malus." Under the generic heading Cydonia, Miller gave references to Cydonia Tourn., and Pyrus of Linnaeus in his Genera Plantarum, 1754. Consequently Pyme Cydonia L. and Cydonia Oblonga Mill. are synonymous in that they are based in part upon the same Tournefort reference. The other references also are accepted as applying to this same species.

The issue as to whether the quince, the pear, and the apple are to be left in a single genus is a taxonomic one. As to the apple and pear, the question is still moot, but there is a consensus of interpretation that the quince forms a separate genus, as can be seen in the manuals, encyclopaedias, and systems of botany. As a genus separated from Pyrus, the first validly published name is Cydonia [Tourn.] Mill. The Index Kewensis gives Miller's Gardeners Dictionary, ed. 6, as the place of origin of this generic name. However, this edition appeared in 1752, a pre-Linnaean date, hence the name is illegitimate, under Art. 13, which, as quoted above, establishes 1 May 1753 as the starting point for scientific botanical nomenclature. The reference given in this case, Miller's ed. 8 , of 1768 , is the first valid publication of a generic name for this group as a genus.

Miller's name Cydonia (Oblonga) foliis oblongo-ovatis, etc., might be considered a polynomial, but examination will show that the latter phrases were the description and the word (Oblonga) was the epithet for the species. It was a better and more economical arrangement with the epithet in parentheses following the generic name, than was Linnaeus' method of plac- 
ing the epithet singly in the margin. That Miller's method was an acceptable indication of a binomial is stated in Art. 32, Examples, "The same result is attained in Miller's Gardeners Dictionary, ed. 8, by the inclusion of the epithet in parentheses immediately after the name of the genus,..." The epithet Oblonga, not being the name of a person, or a vernacular name, or a former generic name, should now be written with small letters, under Rec. $73 \mathrm{~F}$, which states, "All specific and infraspecific epithets should be written with a small initial letter, ..."

Cydonia Cydonia (L.) Pers. was a combination, made by Persoon in 1807, of the oldest specific epithet (1753) under the valid generic name Cydonia (1768). When Persoon did this, the practice was legal. Now, all the recent codes of nomenclature have legislated against this kind of action. The most recent code, that of 1954 (published in 1956) asserts, Art. 70, "epithet is illegitimate ...(4) When it exactly repeats the generic name, ..." These tautonyms are now invalid, so, though the epithet Cydonia of Linnaeus was the first valid name, it cannot be used, as it is illegitimate if combined under the genus Cydonia as Cydonia Cydonia.

The Index Kewensis gives Cydonia vulgaris Pers. as having been published by Persoon on page 40 of his Synopsis. That is incorrect. But C. K. Schneider's Illustriertes Handbuch der Laubholakunde 1: 654, 1906, gives the reference as the Errata in Persoon's Synopsis. That is correct, and there Persoon says, "ob infer. loco Cydonia leg. vulgaris," so Persoon's corrected name for the quince was Cydonia vulgaris (1807). Under the species he gives a description, and a reference to Pyrus L., which ties his new name back to the original Pyrus Cydonia L. By our present rules Persoon was correct in avoiding a tautonym, but he should have adopted the earliest specific epithet, in this case, oblonga of Miller (1768). The two epithets are certainly synonyms, both being traceable back to Linnaeus and to Tournefort. Art. 11 provides, "Each order or taxon of lower rank with a particular circumscription, position, and rank can bear only one correct name,... For any taxon below the rank of genus inclusive, the correct name is the earliest legitimate one validly published with the same rank, except in cases of limitation of priority by conservation ..." Hence, vulgaris of Persoon is illegitimate, oblonga of Miller being earlier and available. 
The correct scientific name of the quince, when classified as a separate genus, is Cydonia oblonga Mill. (1768).

The solution of this nomenclatural case brings the student in contact with several of the fundamental and great botanical books. The correct interpretation of this case rests upon an ability to read scientific books in several languages, an understanding of the principles of priority, synonymy, and the regulations governing the binomial system and tautonyms. Other principles, laws, and recommendations are illustrated by the other cases. Often a case will involve many of the articles and recommendations of the International Code of Botanical Nomenclature.

\section{Useful Publications}

The publications useful to a student of nomenclature are many, too many to be listed here. However, a selection of the most serviceable ones is classified and listed below.

\section{Indexes}

Andrews, H. N., Jr. Index of Generic Names of Fossil Plants, 1820-1950. U. S. Geol. Surv., Bul. 1,013. 1955.

Gray Herbarium card index of new genera, species and varieties of American plants, 1885- .

Merrill, E. D. Index Rafinesquianus. 1949.

\section{PHANEROGAMS}

Index Kewensis 1-4. Supplements 1-11- . 1895- •

\section{FILICINAE}

Christensen, C. Index Filicum, and Supplements. 1906, 1913, $1917,1934$.

\section{MARSILEACEAE AND SALVINIACEAE}

Reed, C. F., Index Marsileata et Salviniata. Sociedade Broteriana, Bol. II, 28: 5-61. 1954.

\section{LYCOPODIUM}

Herter, W. (or Guilemus). Index Lycopodiorum. 1949.

Herter, W. Systema Lycopodiorum. 1949-50. 
ISOËTALES

Reed, C. F. Index Isoëtales. Sociedade Broteriana, Bol. II, 27: 1-72. 1953.

OTHER FERN ALLIES

None.

LICHENS

Zahlbruckner, A. Catalogus Lichenum Universalis, 10 vol. 1921-1940.

FUNGI

Saccardo, P. A. Sylloge Fungorum. 18 vol. 1882-1906.

ALGAE

de Toni, G. B. Sylloge Algarum. 6 vol. 1889-1924.

DESMIDIACEAE

Norstedt, C. F. O. Index desmidiacearum citiationibus locupletissimus atque bibliographia, and supplementum. 1896, 1908.

BACTERIA

None, but see Bergey, D. H. Manual of Descriptive Bacteriology. 1949.

\section{Systems}

Bentham, G., and Hooker, J. D. Genera Plantarum. 3 vol. 18621883.

De Candolle, A. P., and A. Prodromus systematis naturalis regni vegetabilis. $17 \mathrm{vol} ., 4 \mathrm{vol}$. index. 1824-1873.

Engler, A., and Prantl, K. Die Natürliche Pflanzenfamilien. Ed. 1, 23 vol., 1887-1915. Ed. 2, 1924- .

Engler, A. Das Pflanzenreich. 1900- .

Hutchinson, J. The Families of Flowering Plants. 2 vol. 1926, 1934.

Johnson, A. M. Taxonomy of Flowering Plants. 1931. Rendle, A. B. The Classification of Flowering Plants. 2 vols. 1904, 1925.

Warming, E. A Handbook of Systematic Botany. Ed. by M. C. Potter, 1904.

Wettstein, R. Handbuch der Systematischen Botanik. Ed. 4, 2 vols. $1933,1935$. 


\section{Nomenclature Catalogues}

Pfeiffer, L. Nomenclator botanicus. 3 vol. in 4. 1873-1874. Up to the end of 1858 this alphabets all names from those of classes down to those of sections.

Steudel, E. Nomenclator Botanicus. Ed. 1, 1824. Ed. 2, 2 vol s., $1840-41$.

\section{Dictionaries}

Willis, J. C. A Dictionary of the Flowering Plants and Ferns. Gth ed. 1948.

\section{Nomenclature Code}

International Code of Botanical Nomenclature adopted by the Eighth International Botanical Congress. Paris, July, 1954. Edited by J. Lanjouw et al., Regnum Vegetabile 8: 1-338, 1956. Utrecht, I. A. P. T.

International Code of Nomenclature for Cultivated Plants, Regnum Vegetabile 10: 1-28, 1958.

\section{Bibliographies}

Biological Abstracts. 1926-.

Botanical Abstracts. 1918-1926.

Botanisches Centralblatt. 1880-。

British Museum of Natural History, Catalogue of Library. 8 vol., including 3 Supplements. 1903-1940.

International Catalogue of Scientific Literature, Botany. Covering 1900-1913.

Jackson, B. D. Guide to Literature of Botany. 1881.

Just's Botanischer Jahresbericht. 1873-.

Kew. Catalogue of the Library of the Royal Botanic Gardens. Kew Bul. Misc. Inf., Add. Ser. 3. 1899. Suppl. 1919.

Merrill, E. D., and Walker, E. H. Botanical Bibliography of the

Pacific。 U. S. Natl. Herb., Contr. 30 (1). 1947.

Pritzel, G. A. Thesaurus Literaturae Botanicae. Ed. 2. 1871.

Rehder, A. Bradley Bibliography of Woody Plants. 5 vols. 19111918.

Rehder, A. Bibliography of Cultivated Trees and Shrubs. 1949. 
Royal Society Catalogue of Scientific Literature. 19 vols. Covering 1800-1900.

\section{Guide to Floras}

Blake, S. F., and Atwood, A. C. Geographical Guide to Floras of the World. Pt. 1, U. S. Dept. Agric., Misc. Publ. 401. 1942.

\section{Cultivated Plants}

Bailey, L. H. Manual of Cultivated Plants. Rev. ed. 1949.

Bailey, L. H. Standard Cyclopedia of Horticulture. 3 vols. 1928, and later reprintings.

Rehder, A. Manual of Cultivated Trees and Shrubs hardy in North America. Ed. 2. 1940. 


\section{CHAPTER 2}

CASE

1. Sida Holtzii F. Muell. (in herb.) ex E. G. Baker, Jour. Bot.

Brit. For. 30: 325, 1892.*

2. Pleiogynium Solandri (Benth.) Engler in DC., Monogr. Phan.

4: 255, pl. 7, fig. 1-10, 1883.

Spondias Solandri Benth., Fl. Austral. 1: 492, 1863.

S. acida Soland. ex Benth., Fl. Austral. 1: 492, 1863.

Owenia cerasifera F. Muell., Hooker's Jour. Bot. \& Kew

Miscel. 9: 305, 1857.

S. pleiogyna F. Muell., Fragm. Phytogr. Austral. 4: 78, 18631864.

Icica? Timoriensis DC., Prodr. 2: 78, 1825.

P. timoriense (DC.) Leenhouts, Blumea 7: 159, 1952.

3. Phleum pratense L. Race II-P. nodosum L. (Pro specie),

$\beta$ intermedium Nob., Rouy, Fl. de France 14: 49-50,

1913.

P. pratense L., Sp. Pl. 59, 1753.

$P$. nodosum L., Syst. Veg., ed. 10, 871, 1759.

$P$. intermedium Jordan ex F. W. Schultz, Fl. France et

Allemagne, Archives 1: 325, $(1842-1848)=[1854]$.

4. Phyllamphora mirabilis Lour., Fl. Cochinch. 606, 1790. Nepenthes Phyllamphora Willd., Sp. Pl. of L., ed. 4 by Willd., 4: 874, 1805.

N. mirabilis (Lour.) Druce, Bot. Exch. Club Brit., Rept. 1916: 637, 1917.

*Customary abbreviations of the authors' names are used in the bibliographic citations. For books the key words of the titles are used in full or in abbreviation. Magazine titles are also abbreviated, except where it is necessary to cite them more fully. The words "plate," "plates," "tables," "tafeln," etc., are shortened to pl.; figures are shortened to fig. Roman numbers of volumes or plates are changed to Arabic numbers, except where confusion might result, but they are retained for a magazine series number and for the pages of an introduction so numbered. 
N. mirabilis (Lour.) Merr., Interpr. Rumph. Herb. Amboinense 242, 1917.

5. Convolvulus sepium L. $\beta$ Americanus Sims, Curtis's Bot. Mag. 19: pl. 732, 1804.

Calystegia sepium (L.) R. Br. $\beta$ rosea Choisy in DC., Prodr. 9: $433,1845$.

6. Collinsia J. Agardh, Analecta Algol. cent. 5: 77-79, 1899. Collinsia Nutt., Acad. Nat. Sci. Phila., Jour. 1: 190, pl. 9, 1817.

Collinsiae Mack. in Britton \& Brown, Ill. Fl. N. U. S. Can., ed. 2, 1: 353, 1913.

Carex Collinsii Nutt., Gen. N. Am. Pl. 2: 205, 1818.

Collinsonia L., Gen. Pl., ed. 5, 16, 1754.

See Silva, Univ. Calif., Publ. Bot. 25: 284, 1952.

7. Cicuta maculata L., Sp. Pl. 256, 1753.

See Coulter \& Rose, U. S. Natl. Herb., Contr. 7: 97-98, 1900;

Fernald, Rhodora 41: 439-440, 1939.

8. Thelpyteris Schmidel, Icon. Pl., ed. 2, 45, pl. 11, 13, 1762. Dryopteris Adans., Fam. Pl. 2: 21, 1763. Nephrodium Rich., Cat. Jard. Med. Paris, 1801.

See Nakai, Bot. Mag. Tokyo 40: 61, 1926;

Mackenzie, Am. Fern. Jour. 17: 117, 1927;

Fernald \& Weatherby, Rhodora 31: 21-36, pl. 179-180, 1929; also as Gray Herb., Contr. n.s. 83: 21-36, pl. 179-180, 1929.

9. Blechnum orientale L., Sp. Pl. 1,077, 1753.

B. occidentale L., Sp. Pl. 1,077, 1753.

10. Englemannia Gray ex Nutt., Am. Phil. Soc., Trans. II, 7: 343-344, 1841.

Engelmannia T. \& G., Fl. N. Am. 2: 283, 1841. Angelandra Endl., Gen. Pl. Suppl. (Mantissa Bot. Alt.) 3: 69, 1843.

Engelmannia Klotzsch, in Wiegmann's Archiv für Naturgesch. 7: 253, 1841. Engelmannia Pfeiff., Bot. Zeit. 3: 673, 1845.

11. Ceodes Umbellifera J. R. \& G. Forst., Char. Gen. Pl. Mar. Austral. 142, pl. 71, 1776. 
C. umbellata J. R. \& G. Forst. emend. G. Forst., Fl. Ins. Austral. Prodr. 93, 1786.

Pisonia umbellata (J. R. \& G. Forst.) Seem., Bonplandia 10: $154,1862$.

12. Houstonia patens Ell. var. pusilla Gray, Syn. Fl. N. Am. 1(2): 25, 1884.

H. pusilla Schoepf, Reise 2: 306, 1788.

H. pusilla J. F. Gmel., Syst. Nat. of L., ed. 13 by J. F.

Gmel., 2: 236, (1788) =[1791].

H. pusilla Schoepf forma rosea Steyerm., Rhodora 41: 585, 1939.

H. pygmaea C. H. \& M. T. Mueller, Torrey Bot. Club, Bul. 63: 33-34, 1936.

H. Taylorae Fosberg, Field \& Lab. 17: 169, 1949.

Hedyotis ? rosea Raf., Fl. Ludovic. 77, 1817.

13. Brodiaea Orcuttii (Greene) Baker, Gard. Chron. III, 20: 214, 1896.

Hookera Orcuttii Greene, Calif. Acad. Sci., Bul. 2: 138, 1886. H. multipedunculata Abrams, Torrey Bot. Club, Bul. 32: 537, 1905.

14. Wyethia amplexicaulis Nutt., Am. Phil. Soc., Trans. II, 7:

352-353, 1840.

Espeletia amplexicaulis Nutt., Acad. Nat. Sci. Phila., Jour.

7: 38, 1834.

See Weber, Am. Midl. Nat. 35: 441, 1946.

15. Puccinia-Convolvuli (Pers.) Ćast., Obs. Pl. Acot. Urédinées

1: 16, 1842.

Vredo betae $\beta$ Vredo Conuoluuli Pers., Syn. Fung. 221, 1801.

See Jackson, Mycologia 23: 495, 1931;

Rickett, Tax on 4: 185-188, 1955.

16. Cracca L., Gen. Pl., ed. 5, 333, 1754; Sp. Pl. 752, 1753.

Cracca Benth. in Benth. \& Oersted, Kjoeb. Vidensk., Meddel.

1853: 8, 1854.

Cracca L. sensu Ktze., Rev. Gen. 1: 173-176, 1891.

Galega L., Syst. Nat., ed. 10, 2: 1,172, 1759; Gen. Pl., ed.

5, 320, 1754; ed. 6, 384, 1764; Syst. Nat., ed. 13, 2:

496-497, 1770.

Tephrosia Fers., Syn. Pl. 2: 328, 1807. 
Benthamantha Alefeld, Bonplandia 10: 264, 1862.

Brittonamra Ktze., Rev. Gen. 164, 1891.

See Wood, Rhodora 51: 197-199, 1949.

17. Geitonoplesium cymosum (R. Br.) A. Cunn. var. timorense

(Ridley) Schlittler, Bot. Mus. Univ. Zürich, Mitt. 189:

228, 238, 1951.

Luzuriaga cymosa R. Br., Prodr. Fl. Nov. Holl. 282,

1810.

Eustrephus timorensis Ridley in H. O. Forbes, Nat. Wand.

East. Archip. 520, 1885.

18. Convolvulus tiliaefolius Desr. in Lam., Encyc. Méth. Bot. 3:

544, 1789.

Ipomoea grandiflora Lam., Tabl. Encyc. 1: 467, 1791.

Stictocardia tiliaefolia (Choisy) Hallier f., Engl. Bot. Jahrb.

18: 159, 1894.

Convolvulus grandiflomes L. f., Suppl. 136, 1781.

Stictocardia tiliifolia (Desr.) Hallier f. emend. Oostr. \&

Hoogl., Fl. Males. 4: 491, 1953.

See van Ooststroom, Blumea 3: 577, 1940.

19. Elsholtzia Willd. in Roem. \& Usteri, Mag. 4(11): 3, 1790.

Elsbolaia Reichb., Consp. 116, $(1828)=[1829]$.

Elsholtia Koch, Syn. Fl. Germ., ed. 2, 631, 1844.

Elschotaia Brongn., Enum. Gen. Pl. 68, 1843.

Elsholaia Moench, Meth. Pl. Hort. Bot. Marburg, 389, 1794.

Elshotzia Roxb., Fl. Ind., ed. Carey, z: 4, 1832.

Elssholzia Garcke, Fl. Deutschl., ed. 6, 307, 1863; ed. 19, 479, 1903.

Eschscholaia Cham. in Nees, Hor. Phys. Berol. 73, index, 1820.

Eschholzia Cham. in Nees, Hor. Phys. Berol., pl. 15, 1820. Eschholtzia Reichb., Consp. 187, 1828.

Escholtzia Dum., Anal. Fam. P1. 52, 1829.

Eschscholtzia Cham., Linnaea 8: 464, 1833.

Eschsholaia DC., Prodr. 3: 344, 1828.

20. Delisea Lamx., Dict. Sci. Nat. 13: 41-42, 1819.

Delissea Gaud., Bot. Voy. Freyc. Uranie 457, pl. 6, 78,

$(1826)=[1829]$.

Delisea Fée, Essai Cryptog. Écorces, pp. LXIV-LXV, 151, 1824. 
Delitschia Auerswald, Hedwigia 5: 49, 1866.

See.Silva, Taxon 6: 143, 1957.

21. Viola Chamissoniana Ging. var. beta $\mathrm{Hbd}$. ex MacCaughey,

Torreya 18: 5, 1918.

V. Chamissoniana Ging. $\beta$ var. Hbd., Fl. Hawaiian Is. 17, 1888.

22. Scaevola Chamissoniana Gaud. var. typica Hochr., Candollea 5: 295, 1934.

23. Absinthium Tourn., Inst. Rei Herb. 1: 457-459, pl. 260, 1700.

Absinthium [Tourn.] ex L., Syst. Nat., ed. 1: 1735; Gen. Pl., ed. 5, 367, 1754.

Absynthium Gaertn., Meyer \& Scherb., Fl. Wetterau 3: 196, $1801^{\circ}$

Absinthium Gaertn., Fruct. 2: 393, pl. 164, 1791. Absinthium Adans., Fam. Pl. 2: 120, 1763.

Artemisia L. sect. Absinthium Besser ex DC., Fl. France 4:

189, 1805; DC., Prodr. 6: 120, 1837.

See Gray, Syn. Fl. N. Am. 1(2): 369, 1884;

Hall \& Clements, Carnegie Inst. Wash., Publ. 326: 46, 106, 1923.

24. Apium Petroselinum L., Sp. Pl. 264, 1753. A. latifolium Mill., Gard. Dict., ed. 8, Apium No. 3, 1768. A. crispum Mill., Gard. Dict., ed. 8, Apium No. 2, 1768. A. hortense, seu Petroselinum vulgo Bauhin, Pinax 153, 1671.

A. vel Petroselinum crispum Bauhin, Pinax 153, 1671.

A. hortense latifolium Bauhin, Pinax 153, 1671.

Petroselinum hortense Hoffm., Gen. Pl. Umbellif. 163, 166, XXV, pl. 1A, fig. 7, pl. 1B, fig. 4, 1814. $P$. sativum Hoffm., Gen. PI. Umbellif. 177 (in indice), 78, 1814.

P. Petroselinum (L.) Karst., Deutschl. Fl., ed. 2, 2: 394, 1895.

P. crispum (Mill.) A. W. Hill, Kew Hand-List Herb. Pl., ed. 3, 122, 1925.

Apium sativum Hoffm. var. Petroselinum crispum Nym.,

Conspec. Fl. Eur. 2: 309, 1879. 
See Airy-Shaw, Kew Bul. 257, 1938; 168, 1939;

Mansfeld, Fedde Repert. Sp. Nov. 46: 307, 1939;

Hill, Harvard Bot. Mus. Leafl. 10: 163, 1942;

Hara, Bot. Mag. Tokyo 61: 716, 1948;

Furtado, Gardens' Bul. 12: 345, 1949.

25. Euonymus [Tourn.] L., Gen. Pl., ed. 5, 91, 1754.

Evonymus L., Sp. Pl. 197, 1753.

See Rickett, Taxon 4: 185-188, 1955;

Lawrence, Baileya 3: 113, 1955.

26. Jungermannia frondibus supra bipinnatis apice floriferis, foliolis ciliatis L., Sp. Pl. 1, 132, 1753.

27. Leuchorchis straminea (Fern.) Löve, Bot. Notis. 1950(1): 36, 1950.

L. albida (L.) E. Mey. ssp. straminea (Fern.) Löve, Bot. Notis. 1950(1): 36, 1950.

Habenaria straminea Fern., Rhodora 28: 174, 1926.

28. Pelea brownii F. Br., Bishop Mus., Bul. 84: 6, 1931.

29. Acosta Ruiz \& Pavon, Fl. Peruv. Chil., Prodr. 1, pl. 1, 1794. Moutabea Aubl., Hist. Pl. Guian. 2: 679, pl. 274, 1775.

30. Kingdon-Wardia Marquand, Linn. Soc. Lond. Bot., Jour. 48: 207, 1929.

31. Lepturus reptans Guillaumin, Fl. Nouv.-Calédonie 32, 1948.

32. Uvularia pudica (Walt.) Fern., Rhodora 41: 536, 1939. Anonymos pudic. Walt., Fl. Carol. 123, 1788. Uvularia puberula Michx., Fl. Bor.-Am. 1: 199, 1803.

33. Circaea lutetiana L. $\beta$ canadensis L., Sp. Pl. 9, 1753. C. lutetiana canadensis L. ex Michx., Fl. Bor.-Am. 1: 17, 1803.

C. intermedia Ehrh., Beitr. Naturk. 4: 42, 1789.

C. quadrisulcata (Maxim.) Franch. \& Sav. var. canadensis

(L.) Hara, Rhodora 41: 387, 1939.

C. lutetiana L. var. quadrisulcata (Maxim.) Maxim. ex

Aschers. \& Magnus, Bot. Zeit. 28: 783, 1870.

C. latifolia Hill, Brit. Herb. 138, 1756.

C. lutetiana L. forma quadrisulcata Maxim., Prim. Fl. Amur. 106, 1859. 
C. lutetiana L. ssp. quadrisulcata Maxim. ex Aschers. \& Magnus, Bot. Zeit. 28: 787, 1870.

C. mollis Sieb. \& Zucc. var. Maximowiczï Lévl., Bul. GeogBot. (21:) = [22:] 223, 1912.

C. Maximowiczii (Lévl.) Hara, Jour. Jap. Bot. 10: 598, fig. 13, 1934.

34. Platyspermum Hoffm., Gen. Pl. Umbellif. 64, 1814. Platyspermum Hook., Fl. Bor.-Am. 1: 68, pl. 18, fig. B, 1829. Idahoa scapigera (Hook.) Nels. \& Macbr., Bot. Gaz. 56: 474, 1913.

Carex Idahoa L. H. Bailey, Bot. Gaz. 21: 5, 1896.

35. Cotyledon Tourn. ex L., Gen. Pl., ed. 5, 196, 1754; Sp. Pl. 429-430, 1753.

36. Quercus marilandica Muenchh., Hausv. 5: 253, 1770. Q. Marylandica Muenchh. emend. Britton \& Brown, Ill. Fl.

N. U. S. \& Can. 1: 518, fig. 1,235, 1896.

37. Hypochoeris L., Gen. Pl., ed. 5, 352, 1754. Hypochaeris L., Sp. Pl. 810-811, 1753.

38. Potamogeton [Tourn.] ex L., Gen. Pl., ed. 5, 61, 1754. Hydrogeton Lour., Fl. Cochinch. 244, 1790. Patamogeton Honck., Synops. Pl. Germ. 2: 110, 1793. Potamogiton Raf., Med. Repos. New York 5: 354, 1808. Potamogetum Clairv., Man. d'herb. Suisse 34, 44, 1811.

39. Nelumbo lutea (Willd.) Pers., Syn. Pl. 2: 92, 1807. Nelumbium luteum Willd., Sp. Pl. of L., ed. 4 by Willd., 2:

$$
1,259,1799 .
$$

Nymphaea pentapetala Walt., Fl. Carol. 155, 1788. Nymphaea Nelumbo L. $\beta$ L., Sp. Pl. 730, 1753. Nelumbo pentapetala (Walt.) Fern., Rhodora 36: 23-24, 1934. Nelumbo [Tourn.] Adans., Fam. Pl. 2: 73, 76, 1763. Nelumbium Juss., Gen. 68, 1789; ed. 2, 76, 1791.

See Gleason, Phytologia 2: 205-206, 1947.

40. Eustrephus latifolius $\mathrm{R}$. Br. ssp. angustifolius (R. Br.)

Schlittler, Bot. Mus. Univ. Zürich, Mitt. 189: 213, 1951. E. angustifolius R. Br., Prodr. Fl. Nov. Holl. 281, 1810.

41. Spongia oculata L., Sp. Pl. 1,170, 1753.

Spongia oculata L., Syst. Nat., ed. 10, 2: 1,348, 1759. 
Haliclona oculata (Linnaeus). See de Laubenfels, Harvard Mus. Comp. Zool., Bul. 103(1): 9, 1949.

42. Clavaria [Vaill.] ex L., Gen. Pl., ed. 5, 493, 1754.

Clavaria Stackh., Nereis Brit., ed. 2, X, 1816.

Clavaria [Vaill.] ex Fr., Syst. Mycol. 1: 465, 1821.

Gelidium Lamx., Essai Thalass., in Paris Mus. Nat. Hist., Ann. 20: 128-129, 1813.

See Doty, Lloydia 2: 126-127, 1948.

43. Dioscorea affinis alata L., ex Christophersen, Bishop Mus., Bul. 128: 51-52, fig. 7, 1935.

44. Balduina Nutt., Gen. N. Am. P1. 2: 175, 1818.

Baldwinia Nutt. emend. Gray, Syn. Fl. N. Am. 1(2): 302, 1884.

45. Zanthoxylum Kauaense Gray, U. S. Expl. Exped. Bot.

Phanerogamia 15(1): 354, 1854.

Zanthoxylum Kauaiense Gray emend. Hbd., Fl. Haw. Ids. 73, 1888.

Z. Kauaiense Gray emend. Hbd., $\gamma$ Hbd., Fl. Haw. Ids. 74, 1888.

Fagara kauaiense (Gray) Engler, Natür. Pflanzenfam., ed. 2, 19a: 217, 1931.

F. kauaiense (Gray) Engler var. kohuana Skottsb., Göteborg Bot. Trädg., Medded. 15: 381, 1944; on p. 518 as kohuaana.

46. Abies (Canadensis) foliis linearibus obtusiusculis submembranaceis. Mill., Gard. Dict., ed. 8, Abies No. 4, 1768. Pinus glauca Moench, Verzeichn. Baeume Weissenst. 73, 1785.

Picea canadensis (Michx.) Link, Linnaea 15: 524, 1841. Picea canadensis (Mill.) BSP., Prelim. Cat. Anth. Pterid.

New York 71, 1888.

Picea glauca Beissn., Handb. Conif. 59, 1887.

Picea glauca (Moench) Voss, Deut. Dendrol. Gesell., Mitt. 16: 93, 1907.

See Rehder, Rhodora 17: 59-62, 1915; Arn. Arb., Jour. 1: 57, 1919.

47. Asparagus caule herbaceo erecto, foliis setaceis . . Mill., Gard. Dict., ed. 8, Asparagus No. 1, 1768. 
48. Viola Kauaensis Gray, U. S. Expl. Exped. Phanerogamia 15(1): 85, 1854.

V. Kauensis Gray, Am. Acad. Arts Sci., Proc. 2: 325, 1852.

V. Kavaiensis Gray ex Mann, Am. Acad. Arts Sci., Proc. 7: $150,1867$.

V. Kawaiensis Gray ex Drake, Ill. Fl. Maris Pacif. 108, 1890.

V. kauaiensis Gray emend. Skottsb., Göteberg Bot. Trädg., Meddel. 13: 513, 1940

49. Fimbristylis marguesana Steud., Syn. Pl. Glum., ed. 2, 2: $107,1855$.

F. marquesana Steud. emend. B. D. Jackson, Ind. Kew. 4:

1,284, 1895; F. Brown, Bishop Mus., Bul. 84: 104, 1931;

Drake, Fl. Polyn. Fr. 242, 1893.

50. Sidalcea Gray, Am. Acad. Arts Sci., Mem. n.s. 4: 18, 1849

(Pl. Fendler.).

S. secundiflora Greene, Fl. Francisc. 103, 1891; Man. Bot. San Francisc. 65, 1894.

See Rousch, Mo. Bot. Gard., Ann. 18: 118, 138, 1931.

51. Cassia hebecarpa Fern., Rhodora 39: 413, 1937.

C. nictitans L. var. hebecarpa Fern., Rhodora 38: 423, 1936.

52. Juncus fucensis St. John, State Coll. Washington, Bot. Dept.,

Contr. 10: first page, 1928, March 31; British Columbia

Prov. Mus., Rept. for 1927: E 14, 1928, July.

53. Rudbeckia columnifera Nutt., Fraser's Cat. 1813; repr. in

Pittonia 2: 116-119, 1890. See Nutt., Gen. N. Am. Pl.

2: 178-179, 1818.

Rudbeckia columnaris Pursh, Fl. Am. Sept. 575, 1814. Ratibida columnifera (Nutt.) Wooton \& Standl., U. S. Nat.

Herb., Contr. 19: 706, 1915.

Lepachys columnaris (Pursh) T. \& G., Fl. N. Am. 2: 313, 1842.

Rudbeckia columnaris Sims, Curtis's Bot. Mag. 39: pl. 1,601, 1813.

Ratibida columnaris (Sims) D. Don in Sweet, Brit. Fl. Gard.

7: 361, 1838.

Ratibida sulcata Raf., Am. Month. Mag. 2: 268, 1818; Jour. Phys. 89: 100, 1819. 
Obeliscaria columnaris (Pursh) DC., Prodr. 5: 559, 1836.

Obelisteca pinnata (Vent.) Raf., Fl. Ludovic. 73, 1817.

See Shinners, Rhodora 57: 290-293, 1955;

Graustein, Rhodora 58: 20-22, 1956;

Cronquist et al., Rhodora 58: 23-24, 1956;

Shinners, Rhodora 58: 281-289, 1956;

Cronquist, Rhodora 59: 100, 1957.

54. Eucanna Baker, Gard. Chron. 43, 1893; Engler \& Prant1, Nat.

Pflanzenfam., ed. 2, 15a: 653, 1930.

Trialatae Kränzl., Engler's Pflanzenreich IV, fam. 47: 47,

1912.

Glaucae Kränzl., Engler's Pflanzenreich IV, fam. 47: 47-48, 1912.

55. Greyiaceae Hutchins., Fam. Fl. Pl. Dicots. 1: 202, 1926. Greyieae Engler \& Prantl, Nat. Pflanzenfam. IV, 3(5): 382, 1896.

Greyia Hook. \& Harvey, Dublin Univ. Zool. Bot. Assoc.,

Proc. 1: 137, pl. 13-14, 1858.

Graya Arn. ex Steud., Nom., ed. 2, 1: 705, 1840.

Graya Endl., Gen. Suppl. 1: 1,376, [1841].

Graya Arn. ex Steud., Syn. Pl. Gram. 119, 1855.

Grayia H. \& A., Bot. Beechey Voy. 387, (1832) =[1841].

Grayia Grove \& Brun in A. Schmidt, Atlas der Diatomaceenk. pl. 172, fig. 11, 1892.

56. Se suvium Portulacastrum L., Syst. Nat., ed. 10, 1,058, 1759;

Sp. Pl., ed. 2, 1: 684, 1762; ed. 3, 1: 684, 1764.

Portulaca Portulacastrum L., Sp. Pl. 446, 1753.

Portulacastrum Juss. ex Medic., Phil. Bot. 1: 99, 1789.

57. Arabis laevigata (Muhl.) Poir. var. heterophylla Farw., Mich.

Acad. Sci., Ann. Rept. 19: 248, 1917.

A. viridis Harger var. Deamii Hopkins, Rhodora 39: 160,

1937.

A. viridis Harger var. heterophylla (Farw.) Farw., Rhodora

41: 80, 1939.

A. missouriensis Greene var. Deamii (Hopkins) Hopkins,

Rhodora 45: 269, 1943.

58. Rosales Engler, Natürl. Pflanzenfam., Nachtr. 348, 1897. 
59. Conyzopsis T. \& G., Fl. N. Am. 2: 162, 1841.

Conyzopsis (T. \& G.) Gray, Am. Acad. Arts Sci., Proc. 16: 99, 1881.

60. Daboecieae Cox, Am. Midl. Nat. 39: 238-239, 1948.

61. Berberis simplex Newberry, U. S. Natl. Mus., Proc. 5: 514, 1883.

Odostemon simplex (Newberry) Cockerell, Am. Mus. Nat.

Hist., Bul. 24: 91, 1908.

Mahonia simplex (Newberry) Arnold, Univ. Mich., Mus.

Paleont., Contr. 5: 58, pl. 1, fig. 1-3, 6-7; pl. 2, fig. $1-2,1936$.

62. Yucca glauca Alliance, J. M. Webber, U. S. Dept. Agric., Monogr. 17: 55, 1953.

63. Claytonia Washingtoniana Suksd., Deutsch. Bot. Monatschr. 16: 220, 1898.

Montia Washingtoniana Suksd., Deutsch. Bot. Monatschr. 16:

220, 1898.

64. Aster nemoralis Ait., Hort. Kew. 3: 198, 1789.

65. Bucklandia Presl, in Sternberg, Versuch Bot. Darstell. Fl.

Vorwelt xxxiii, 1825 $=$ [1820].

Bucklandia R. Br., in Wall. Cat. (Num. List Pl. E. Ind.) No.

7,414, 1832.

Symingtonia van Steenis, Bot. Néerl., Acta 1: 443-444,

1952.

See Taxon 3: 221, 1954.

66. Liparis Yamadae Tuyama, Bot. Mag. Tokyo 54: 265, pl. 2n., 1940.

Cestichis Yamadae Tuyama, Bot. Mag. Tokyo 54: 265, pl. 2n., 1940.

67. Amarella quinquefida Gilib., Fl. Lith. Inch. 1: 36, 1781. Gentiana Amarella L., Sp. Pl. 230, 1753.

68. Gentiana Centaurium L., Sp. Pl. 229, 1753.

Centaurium minus Garssault, Fig. Pl. Anim. Med. 137, pl. 206, 1764.

Erythraea Centaurium (L.) Pers., Syn. Pl. 1: 283, 1805. Centauriom Centaurium (L.) W. F. Wight, U. S. Natl. Herb.,

Contr. 11: 449, 1906. 
Centaurium minus Moench, Meth. Pl. Hort. Bot. Marburg 449, 1794.

Centaurium umbellatum Gilib., Fl. Lith. 1: 35, 1785.

Centaurium Erythraea Rafn, Danm. og Holst. Fl. 2: 70, 1800. Chironia Centaurium (L.) F. W. Schmidt, Fl. Boem. 2: 31,

1793.

See Britten \& Rendle, Jour. Bot. Brit. For. 47: 322, 1909; Jonker, Nederl. Kruidk., Arch. 57: 184-188, fig. la, 1950; repr. as Bot. Mus. Herb. Rijksuniv. Utrecht, Meded. 99: 184-188, fig. la, 1950;

A. Robyns, Jard. Bot. État Bruxelles, Bul. 24: 357367, 1954.

69. Achras Zapota L., Sp. Pl. 1,190, 1753.

A. Zapota $\beta$ Achras Zapotilla Jacq., Select. Stirp. Am. Hist. 57, pl. 41, 1763.

Sapota Achras Mill., Gard. Dict., ed. 8, Sapota No. 1, 1768. Achras zapotilla (Jacq.) Nutt., N. Am. Sylva 3: 28, 1849. Manilkara zapotilla (Jacq.) Gilly, Yale Univ. School For.

Trop. Woods 73: 20, 1943.

See Lam, Trav. Bot. Néerl., Rec. 36: 509-525, 1939;

Baehni, Candollea 9: 147-476, 1942;

Gilly, Trop. Woods, 73: 1-22, 1943;

Cronquist, Lloydia 9: 241-292, 1946;

Lawrence, Gent. Herb. 8: 61, 1949;

Monachino, Phytologia 4: 94-118, 1952;

Little, U. S. Dept. Agric., Agric. Handbook 41: 43-44, 1953.

70. Aspergillus [Micheli] ex Fries, Syst. Mycol. 3: 383-385,

1829.

Eurotium Link ex Fries, Syst. Mycol. 3: 331, 1832.

See Fisher, in Engler \& Prantl, Natürl. Pflanzenfam. 1(1):

52, 58, 301-304, 1896.

Thom \& Raper, Man. Aspergilli 1945.

71. Cyrtophyllum ? lanceolatum A. DC., Prodr. 9: 31, 1845.

72. Pinus ball Brown, U. S. Geol. Surv., Prof. Pap. 185: 53, pl. 8, fig. 5, 1934.

P. balli Brown emend. Brown, Wash. Acad. Sci., Jour. 30:

354, 1940.

See Lamotte, Geol. Soc. Am., Mem. 51: 253, 1952. 
73. Rollandia lanceolata Gaud., Bot. Voy. Freyc. Uranie 458, $(1830)=$ [1829]; Atlas, pl. 74 (as R. montana), 1826. Lobelia lanceolata (Gaud.) Endl., Wien Mus. Naturgesch., Ann. 1: 170, 1836.

R. lanceolata Gaud. var. grandifolia DC., Prodr. 7: 344, 1838.

R. lanceolata Gaud. var. typica Rock, Bishop Mus., Mem. 7(2): 371-373, pl. 209, 1919.

R. lanceolata Gaud. var. Rockii St. John \& Hosaka, Bishop

Mus., Occ. Pap. 11: 13, 1935.

See Fosberg, Am. Jour. Bot. 26: 229, 1939;

St. John, Bishop Mus., Occ. Pap. 15: 236, 1940.

74. Fraxinus pubescens Lam. $\delta$ subpubescens (Pers.) Pursh, Fl. Am. Sept. 1: 9, 1814.

F. pubescens* subpubescens Pers., Syn. Pl. 2: 605, 1807. $F$. juglandifolia Lam. $\beta$ subintegerrima Vahl, Enum. Pl. 1: 50-51, 1804.

F. lanceolata Borkh., Handb. Forstbot. Forsttechn. 1: 826$827,1800$.

$F$. caroliniana Mill. $\beta F$. latifolia Willd., Sp. Pl. of L., ed. 4 by Willd. 4: 1,103, 1806.

F. expansa Willd., Berl. Baumzucht, ed. 2, 150, 1811.

F. viridis Michx., Hist. Arb. For. Am. Sept. 3: 115-117, pl.

10, 1813.

F. pennsylvanica Marsh. var. lanceolata (Borkh.) Sarg., Silva 6: 50-51, pl. 122, 1894.

F. pennsylvanica Marsh. var. subintegerrima (Vahl) Fern.,

Rhodora 49: 159, 1947.

75. Aira L., Gen. Pl., ed. 5, 31, 1754.

Aspris Adans., Fam. Pl. 2: 496, 522, 1763.

Deschampsia Beauv., Agrost. 91, pl. 8, fig. 3, 1812.

See Hitchcock, U. S. Dept. Agric., Bul. 772: 114-116, 1920.

76. Clerodendron aculeatum (L.) Griseb. var. gracile Griseb. \& Moldenke, in Moldenke, Geogr. Distrib. Verbenac. 5-7, 14, 36, 1939.

Clerodendrum aculeatum (L.) Schlecht. var. gracile Griseb. \& Moldenke, in Moldenke, Geogr. Distrib. Verbenac. 29, 43, 46, 47, 158, 180, 1939. 
Clerodendrum aculeatum (L.) Schlecht. var. gracile Griseb. \& Moldenke ex Moldenke, Some New Species and Vars. Verbenac. 13, n.d. [1940].

77. Passiflora edulis Sims var. flavicarpa C. D. Miller, Bazore \& Robbins, Hawaii Agric. Exp. Sta., Bul. 77: 66, fig. 9, 1934.

P. edulis Sims forma flavicarpa Degener, Fl. Haw. 250:

$7 / 30 / ' 32$.

P. edulis flavicarpa W. T. Pope, Hawaii Agric. Exp. Sta., Bul. 74: 13, 1935.

P. edulis Sims var. flavicorpa C. D. Miller \& Bazore, Hawaii Agric. Exp. Sta., Bul. 96: 97, fig. 14, 1945.

78. Mentha gentilis L., Sp. Pl. 577, 1753.

Mentha arvensis $\times$ viridis $\mathrm{F}$. W. Schultz, Pollichia, Jahresber.

12: 42, 1854.

M. Wirtgeniana F. W. Schultz, Pollichia, Jahresber. 12: 41, 1854.

79. Pfeifferago Ktze., Rev. Gen. Pl. 1: 227, 1891. Codia J. R. \& G. Forst., Char. Gen. 59-60, pl. 30, 1776.

80. Caladium Vent. in Roem., Arch. Bot. 2: 347-351, 1800. Calladium acre R. Br., Prodr. Fl. Nov. Holl. 336, (1799)= [1810].

Colocasia esculenta (L.) Schott in Schott \& Endl. var. acris

(R. Br.) A. F. Hill, Harvard Univ. Bot. Mus. Leafl. 7: $117,1939$.

Colocasia acris (R. Br.) Schott in Schott \& Endl., Melet. Bot. 1: $18,1832$.

81. Anemone quinquefolia L. var. interior Fern., Rhodora 37: 260, 1935.

A. quinquefolia L. var. bifolia Farw., Mich. Acad. Sci. Arts Lett., Pap. 1: 94, 1923.

82. Platyschkuhria (Gray) Rydb., Torrey Bot. Club, Bul. 33: 154, 1906.

Cordylophorum Rydb., Fl. Rocky Mts., 590, 1,064, (1917)= [1918].

Pycnosphace Rydb., Fl. Rocky Mts., 747, 1,066, (1917)= [1918]. 
83. Gymnobythus (A. DC.) Gray, Am. Acad. Arts Sci., Proc. 10: $321,1875$.

Gymnobythus A. DC., in DC., Prodr. 9: 296, 1845.

84. Anonymos (Lupino affinis) rotundifolia Walt., Fl. Carol. 181, 1788.

Crotalaria rotundifolia Poir. in Lam., Encyc. Méth. Bot.

Suppl. 2: 402, $(1811)=[1812]$.

See Fernald \& Schubert, Rhodora 50: 202-203, 1948.

85. Abrotanum Tourn., Inst. Rei Herb. 1: 459, 1700.

Abrotanum L., Syst. Nat., ed. 1, 1735; repr. in Jour. Bot.

Brit. For. 38: 440, 1900.

Artemisia L. sect. Abrotanum (Necker) Bess., Soc. Nat.

Mosc., Nov. Mem. 3: 21, 1834.

Euartemisia Gray, Syn. Fl. N. Am. 1(2): 369, 1884.

See Hall \& Clements, Carnegie Inst. Wash., Pubi. 326: 46, 49, 1923.

86. Lobelia glandulosa Walt. $y$ obtusifolia DC., Prodr. 7: 378, 1839.

L. Puberula Michx. var. a glabella Elliott, Sketch Bot. S. Carol. 1: 267, $(1821)=[1817]$.

L. puberula Michx. $\beta$ glabella Hook., Curtis's Bot. Mag. 61: pl. 3,292, 1834.

L. puberula Michx. var. obtusifolia (A. DC.) Fern., Rhodora 49: 184, 1947.

87. Cyrtandra triflora Gaud. $\beta$ var. grandifolia Hbd., Fl. Haw. Is. 332, 1888.

C. grandifolia Elmer, Leafl. Philipp. Bot. 7: 2,663, 1915.

C. laxiflora Mann var. grandifolia (Hbd.) Rock, Am. Jour. Bot.

6: 207-208, 1919.

C. laxiflora Mann var. rhizantha Rock, Am. Jour. Bot. 6: 207, 1919.

C. rhizantha Kränzlin, Linn. Soc. Lond. Bot., Jour. 37: 277, 1906.

C. stupantha St. John \& Storey, Bishop Mus., Occ. Pap. 20: $88,1950$.

88. Delphinium Nuttallii Gray, Bot. Gaz. 12: 51, 54, 1887. D. Nuttallianum Pritz. ex Walpers, Repert. Bot. Syst. 2:

Suppl. 1: 744, 1843. 
89. Dryopteris Linnaeana C. Chr., Ind. Fil. 275, 1906.

Polypodium Dryopteris L., Sp. Pl. 1,093, 1753.

Polystichum Dryopteris (L.) Roth ex Mertens, Röm. Arch.

Bot. 2(1): 106, 1799.

Polystichum Dryopteris (L.) Roth, Tent. Fl. Germ. 3(1): 8082, 1800.

Nephrodium Dryopteris (L.) Michx., Fl. Bor.-Am. 2: 270, 1803.

Filix pumila Gilib., Exer. Phytol. 2: 558, 1792.

Polypodium pulchellum Salisb., Prodr. Stirp. Hort. Allerton 404, 1796.

Lastraea Dryopteris (L.) Bory, in Dict. Class. 9: 233, 1826. Aspidium Dryopteris (L.) Baumg., Enum. Stirp. Transsil. 4:

29, 1846.

Phegopteris Dryopteris (L.) Fée, Gen. Fil. (Mem. Foug. 5:) 243, 1850-1852.

Phegopteris Dryopteris (L.) Fée disjuncta Trel., Harrim.

Alaska Exped. 5: 382, 1904.

Gymnocarpium Dryopteris (L.) Newm., Phytol. 4: 371, 1851. Dryopteris pulchella (Salisb.) Hayek, Fl. Steiermark 1: 39,

1908.

Thelypteris Dryopteris (L.) Slosson in Rydb., Fl. Rocky Mts. 1,044, 1,069, 1917.

Dryopteris triangularis (St. Lager) Herter, Herb. Boiss., Bul.

II, 8: 797, 1908.

Dryopteris disjuncta (Rupr.) C. V. Morton, Rhodora 43: 217, 1941.

Polypodium Dryopteris L. $\gamma P$. disjunctum Rupr., Distr. Cr.

Vasc. Ross. 52, 1845; Pflanzenk. Russ. Reich, Beitr. 3:

52,1845 .

Polypodium disjunctum Rupr. ex Schur, Oestr. Bot. Zeitschr.

8: $193,1858$.

Polypodium Dryopteris L. $\gamma$ disjunctum (Rupr.) Ledeb., Fl. Ross. 4: 509, 1853.

Dryopteris Linnaeana C. Chr. var. disjuncta (Rupr.) Fomin, Fl. Siberia et Orient Extr. 79, 1930.

Gymnocarpium Dryopteris (L.) Newm. var. disjunctum (Rupr.) Ching, Biol. Lab., Sci. Soc. China, Bot. Ser., Contr. 9: 41, 1933. 
90. Rubus Grimesii Bailey, Gent. Herb. 2: 331, fig. 158, 1932;

5: 406, fig. 184, 1943; 7: 494-495, 1949.

See Fernald, Rhodora 50: 79, 1948.

91. Charpentiera obovata Gaud., Bot. Voy. Freyc. Uranie 444, $(1826)=$ [1829]; Alt. 500-600 hexap.

Viburnum nudum L. var. angustifolium T. \& G. See Fernald,

Rhodora 49: 93, 1947, flowering but only 3 or 4 feet

high, ...

Paspalum setaceum Michx. var. calvescens Fern., Rhodora

49: 121, 1947, about 1/2 mile west of Kilby, Virginia.

92. Diellia Brownii E. Br., Bishop Mus., Bul. 89: 46, 1931.

93. Argyroxiphium sandwicense DC. See Hbd., Fl. Haw. Is. 219, 1888, Ahinahina.

94. Vrydagzynea Bl., Coll. Orch. Archip. Ind. 71-72, pl. 17 E-F; 19, fig. 2-3; 20, fig. 1-4, 1858.

95. Nama Sandwicensis Gray, Am. Acad. Arts Sci., Proc. 5: 338, 1861.

N. sandvicense Gray emend. Brand, in Engler, Pflanzenreich IV, fam. 251: 152, 1913.

See C. L. Hitchcock, Am. Jour. Bot. 20: 415-534, 1933.

96. Durio Adans., Fam. Pl. 2: 399, 1763.

97. Geniostoma J. R. \& G. Forst., Char. Gen. 23-24, pl. 12, 1776.

98. Polypodium L., Gen. Pl., ed. 5, 485, 1754; Sp. Pl. 1,082$1,094,1753$.

Polypodium L. emend. C. Chr., Ind. Fil. 506-575, 1906. Polypodium L. emend. Copel., Gen. Fil. 180-181, 1947.

99. Heteropogon Pers., Syn. Pl. 2: 533, 1807.

100. Artocarpus J. R. \& G. Forst., Char. Gen. Pl. Mar. Austral. 101-102, pl. 51, 51a, 1776.

Artocarpus Forst. f., Fl. Ins. Austral. Prodr. 64, 1786. Radermachia Thunb., Vet. Akad. Stockh., Handl. 37: 250255, 1776.

101. Oryzopsis Michx., Fl. Bor.-Am. 1: 51, pl. 9, 1803.

102. Solanum vulgare L., Sp. Pl. 186, 1753. S. vulgare Hegetschw., ed. Heer, Fl. Schweiz, 219, 1840. 
103. Rhus Coriaria L., Sp. Pl. 265, 1753.

R. javanica L., Sp. Pl. 265, 1753.

R. angustifolium L., Sp. Pl. 267, 1753.

R. radicans L., Sp. Pl. 266, 1753.

R. Toxicodendr L., Sp. Pl. 266, 1753.

R. Copallinum L., Sp. Pl. 266, 1753.

104. Salix Hippophaefolia Thuill., Fl. Env. Paris, ed. 2, 514515, (An VII) $=$ [1799].

105. Elaeagnus argenteus Nutt., Fraser's Cat. 1813; repr.

Pittonia 2: 117, 1890.

E. argentea Moench, Meth. Pl. Hort. Marburg 638, 1794.

Elaeagmus argentea Pursh, FI. Am. Sept. 114, 1814.

Elaeagnus argentea Colla, Hort. Ripul., pl. 28, 1824.

E. commutata Bernh., Allg. Thuer, Gartenz. 2: 137, 1843.

Elaeagrus Pallas, Fl. Ross. 1(1): 10=11, pl. 4-5, 1784.

See Shinners, Rhodora 57: 290-293, 1955;

Graustein, Rhodora 58: 20-22, 1956;

Cronquist et al., Rhodora 58: 23-24, 1956; 58: 281-289, 1956.

Cronquist, Rhodora 59: 100, 1957.

106. Rosa lapwaiensis St. John, Fl. S. E. Wash. \& Adj. Ida. 208209, 1937.

107. Carex chlorocystis Böck. in Engl. Bot. Jahrb. 5: 520, 1884.

108. Cardamine Matthioli Moretti ex Comol., Fl. Com. 5: 157-

159, 1847.

109. Eleocharis R. Br., Prodr. Fl. Nov. Holl. 224, 1810. Heleocharis R. Br. emend. Lestib., Essai Cypérac. 22, 41, 1819.

110. Carex Mühlenbergii Willd., Sp. Pl. of L., ed. 4 by Willd., 4: 231, 1805.

C. Muhlenbergii Schk. sensu Brongn., Voy. Duperr. Bot. 2: $151,1829$.

C. Muhlenbergii Kunth ex Boott, Ill. Gen. Carex 3: 125, 1862.

111. Micranthemum Michx., Fl. Bor.-Am. 1: 10, pl. 2, 1803.

112. Brosimum parinarioides Ducke, Jard. Bot. Rio de Janeiro, Arch. 3: 23-30, 1922.

113. Aecidium Gaurae Ellis \& Everh., Erythea 1: 205, 1893. 
114. Carex chlorantha R. Br., Prodr. Fl. Nov. Holl. 242, 1810.

115. Psychotria Taupotinii F. Br., Bishop Mus., Bul. 130: 313315, fig. 54, 1935.

116. Gaura mollis Nutt. ex. T. \& G., Fl. N. Am. 1: 519, 1840.

117. Seemann, B., Fl. Vit. 1-453, 100 pl., 1865-1873.

118. Aplopappus Cass., Dict. Sci. Nat. 56: 168-170, 1828. Haplopappus Cass. ex Endl., Gen. Pl. 385-386, (1840)= [1837].

Diplopappus Less., Soc. Philom., Bul. Sci. 1817: 137, 1817.

119. Wawra, H., Beiträge zur Flora der Hawai'schen Inseln 1158, n. d.

120. Stellandria Brickell, Med. Repos. New York 6: 327, 1803.

121. Pinus Murrayana Oregon Committee, Bot. Exped. Oreg.

[Rept. 8:] 2, No. 740, pl. [4], [1853].

See Murray, Edinb. Bot. Soc., Trans. 6: (225) 350, 1860.

Little, Am. Jour. Bot. 31: 588-590, 1944.

122. Gilia (Dactylophyllum) Parryae Gray, Am. Acad. Arts Sci., Proc. 12: 76-78, 1876.

123. Hyssopus [Tourn.] ex L., Gen. Pl., ed. 5, 248, 1754.

124. Bebbia Greene, Calif. Acad. Sci., Bul. 1: 179, 1885. Kuhnioides Gray, Syn. Fl. N. Am. 1(2): 113, 1884.

125. Harrimanella Cov., Wash. Acad. Sci., Proc. 3: 570, 574576, fig. 62, 1901.

Andromeda L., Gen. Pl., ed. 5, 186, 1754.

126. Canavalia pubescens H. \& A., Bot. Beechey Voy. 81, 1832. C. galeata Gaud. var. pubescens (H. \& A.) Gray, Bot. U. S.

Expl. Exped. 15(1): 441, 1854.

C. galeata (Gaud.) H. \& A. var. pubescens (H. \& A.) St.

John, Bishop Mus., Occ. Pap. 15(22): 231-233, 1940.

127. Kosteletakya Presl, Rel. Haenk. 2: 130, pl. 70, $(1831)=$ [1835].

128. Railliardia Gaud., Bot. Voy. Freyc. Uranie 469, $(1826)=$ [1830].

Raillardia Gaud., Bot. Voy. Freyc. Uranie Atlas, pl. 83, 1826. 
Dubautia Gaud., Bot. Voy. Freyc. Uranie 469, (1826)= [1830]; Atlas, pl. 84, 1826.

See Keck, Bishop Mus., Occ. Pap. 11(19): 24-28, 1936.

129. Crypsinus Presl, Epim. Bot. 123, 1849. See Copel., Gen.

Fil. 205, 1947.

Microterus Presl, Epim. Bot. 124, 1849.

Phymatopsis J. Sm., Hist. Fil. 104, 1875.

130. Penaea L., Gen. Pl., ed. 5, 50, 1754.

131. Amsinckia Lemmonii Macbr., Gray Herb., Contr. n.s. 48: 50-51, 1916.

132. Chrysothamnus (Nutt.) Gray, Am. Acad. Arts Sci., Proc. 8: $642,1873$.

Chrysothamnus Nutt., Am. Phil. Soc., Trans. II, 7: 323, 1841.

133. Brassia R. Br. in Ait., Hort. Kew., ed. 2, 5: 215, 1813.

134. Bamia Manihot (L.) R. Br. ex Sims, Curtis's Bot. Mag. 41:

pl. $1,702,1815$.

B. Manihot (L.) Wall., Cat. (Numer. List Pl. E. Ind.), No. $1,926,1829$.

Bamia [L.] R. Br. ex Wall., Pl. Asiat. Rar. 1: 39, 1830.

Bammia Rupp., Fl. Ienensis 31, 1726; and ed. Haller, 39-40, 1745.

135. Monophyllaea R. Br. in Benn. Pl. Jav. Rar. 121, 1838-1852.

136. Euptelea Sieb. \& Zucc., Fl. Jap. 1: 133, pl. 72, 1835.

137. Pinus resinosa Ait., Hort. Kew. 3: 367, 1789.

138. Cocos nucifera L., Sp. Pl. 1,188, 1753.

139. Oxytropis johannensis (Fern.) Fern., Rhodora 30: 145, 1928. O. campestris (L.) DC. var. johannensis Fern., Rhodora 1:

$88,1899$.

Carex katahdinensis Fern., Rhodora 3: 171, 1901. Melica Nebrodensis Parl., Giorn. Bot. Ital. 2(1): 198, 1844.

140. Aster pseudo-Asa-Grayi Makino, Jour. Jap. Bot. 8(2): 14, 1932; Hara, Enum. Sperm. Jap. 2: 135, 1952. Aster Asa-Grayi Makino, Bot. Mag. Tokyo 22: 157, 1908.

141. Panicum ciliatiflomum Wood, Class-book 2: 786, 1861. $P$. ciliatifolium Kunth, Distrib. Meth. Gram. 1: 36, $(1835)=$ [1829]. 
142. Betula coemulea Blanchard, Betula 1: one page, unnumbered, 1904.

B. $\times$ caerulea Blanchard (pro specie), Little, Check List

Nat. Trees U. S., For. Serv., U. S. Dept. Agric. 30, 1944.

143. Anaphalis margaritacea (L.) B. \& H., Gen. Pl. 2: 303, 1873. Gnaphalium margaritaceum L., Sp. Pl. 850, 1753. Antennaria margaritacea (L.) R. Br., Linn. Soc. Lond.,

Trans. 12: 123, 1817.

Anaphalis margaritacea (L.) Benth. ex C. B. Clarke,

Compos. Indicae 103, 1876.

Anaphalis margaritacea (L.) C. B. Clarke in Hook. f., Fl.

Brit. Ind. 3: 282, 1881.

144. Baileya Kuetz., Tab. Phyc. 7: 35, pl. 87, fig. 3, 1857, non Harv. \& Gray in Torr. (1848).

145. Polypodium vulgare Auct. east. Am., non L., Sp. Pl. 1,085, 1753.

P. virginianum L., Sp. PI. 1,085, 1753.

See Fernald, Rhodora 24: 125-142, 1922.

146. Zingiber Boehm. in Ludw., Def. Gen. Pl., ed. 3 by Boehm. 89, 1760.

Zingiber Mill., Gard. Dict., abridg. ed. 4, 3: under letter Z, 1754.

Zinziber P. Browne, Civ. Nat. Hist. Jamaica 119, 1756. Zingiber Adans., Fam. Pl. 2: 66, 1763.

Dymczewiczia Horan., Prodr. Scitam. 26, 1862.

Lampujang Koen., in Retz., Obs. Bot. 3: 62, 1783.

$Z$ erumbet Lestib., Ann. Sci. Nat. Bot. II, 15: 329-331, pl.

21, fig. 13-17, 1841.

147. Parnassia Turcaaninowii Ledeb., Fl. Ross. 1: 263, 1842.

148. Asplenium platyneuron (L.) Oakes in Eaton, Ferns N. Am.

1: $24,1878$.

Acrostichum platyneuros L., Sp. PI. 1,069, 1753.

See Fernald, Rhodora 37: 382-384, 1935.

149. Palmocarpon Lesquereux, U. S. Geol. Surv., Terr. Rept. 7: $119,1878$.

P. compositum (Lesq.) Lesq., U. S. Geol. Surv., Terr. Rept. 7: 119, pl. 11, fig. 4, 1878. 
Carpolithes compositus Lesq., U. S. Geol. Surv. Terr., Fifth Ann. Rept. for 1871: Suppl. 16-17, 1872.

150. Asplenium $\times$ Souchei R. de Litard., Soc. Bot. Deux-Sèvres, Bul. 21: 100, pl. 1, 1910.

151. Muscites Brongn., Hist. Vég. Fossiles 93, pl. 10, fig. 1-2, 1828.

Muscites florissanti (Knowlton) Steere, Am. Midl. Nat. 36: 310, 1947.

Polytrichum (?) florissanti Knowlton, U. S. Natl. Mus., Proc. 51: 245, pl. 12, fig. 4, 1916.

152. Pyrus hybrida Moench, Verz. Ausland. Bäume u. Stauden 90, pl. 6, 1785. Aronia hybrida (Moench) Zabel in Beissner, Schelle \& Zabel, Handb. Laubholz-Benennung 193, 1903. $\times$ Sorbaronia Schneid., Fedde Repert. Sp. Nov. 3: 134, 1906. $\times$ Sorbaronia monstrosa (Zabel) C. Schneid., Fedde Repert.

Sp. Nov. 3: 134, 1906.

Aronia monstrosa (Hort.) Zabel in Beissner, Schelle \& Zabel, Handb. Laubholz-Benennung 677, 1903.

153. Cactus ferox Nutt. in Fraser's Cat. 1813; repr. in Pittonia 2: $116,1890$.

C. ferox Nutt., Gen. N. Am. Pl. 1: 296, 1818.

Opuntia missouriensis DC., Prodr. 3: 472, 1828. C. ferox Willd., Enum. Pl. Hort. Berol., ed. Schlecht.,

Suppl. 35, 1813.

Opuntia polyacantha Haw., Suppl. Pl. Succ. 82, 1819. Mammillaria Haw., Syn. Pl. Succ. 177, 1812.

Cactus L., Gen. Pl., ed. 5, 210, 1754; Sp. Pl. 466-470, 1753.

See Britton \& Rose, Carnegie Inst. Wash., Publ. 248(1): 199, 1919;

Shinners, Rhodora 57: 290-293, 1955;

Graustein, Rhodora 58: 20-22, 1956;

Cronquist et al., Rhodora 58: 23-24, 1956;

Shinners, Rhodora 58: 281-289, 1956;

Cronquist, Rhodora 59: 100, 1957.

154. Delissea Kunthiana Gaud., Voy. Freyc. Bonite, Atlas, pl. 77, 1844. 
Cyanea Kunthiana Hbd., Fl. Haw. Is. 264, 1888.

Cyanea Bonita Rock, Bishop Mus., Mem. 7(2): 51, 1919.

C. Bishopii Rock, Bishop Mus., Mem. 7(2): 277-279, pl. 52, 155, 1919.

155. Sm. $=$ Smith, Sir James Edward (1759-1828). Consider abbreviations for Albert Charles (1906- ); Annie Lorrain (1854-1937); Arlow Irving (1911- ); Erwin Frink (1854-1927); Gilbert Morgan (1885- ); Jared Gage (1866-1957); Johannes Jacobus (1867-1947); John (1798-1888); John Donnell (1829-1928); Karl August Harald (Harry) (1889- ); Lyman Bradford (1904); William (1808-1857); Sir William Wright (1875-1956).

156. Elymotrigia Hyl., Bot. Notis. 358, 1953.

157. F. Muell. =Sir Ferdinand Jacob Heinrich von Mueller (18251896). Consider abbreviations for Carl (1817-1870); Carl Alfred Ernst (1855-1907); Ferdinand (another publishing in 1852-1862); Franz (publishing 1829-1859); Jean (18281896); Jean Baptista (1806-1894); Johann Karl August (1818-1899); Johann Sebastian (later John) (1715?-1790?); Joseph (published from 1832-1859); Otto Frederik (17301784); Philipp Jakob (1832-1889).

See Eardley, Australasian Herb. News 12: 3-4, 1953.

158. Dicranum (Scopario-Dicranum) yakushimense Sak., Jour. Jap. Bot. 27: 155, 1952.

159. Nothofagus Bl., Mus. Bot. Lugd.-Bat. 1: 307, 1850. Fagaster Spach, Hist. Nat. Vég. Phan. 11: 142, 1842. Calucechinus Hombr. \& Jacq. in Dumont d'Urville, Voy. Pol Sud Astrolabe Zélée Bot. Atlas, Dicot. 19, pl. 6, $7 \Sigma, 1844 ; 8 \pi 60,1845$. Calusparassus Hombr. \& Jacq. in Dumont d'Urville, Voy. Pol Sud Astrolabe Zélée Bot. Atlas Dicot., pl. $6 \Sigma$, 1844; pl. 7 Г, 1844; $8 \Psi, 1845$.

160. Nomosa I. M. Johnston, Jour. Arn. Arb. 35: 24-27, 1954.

161. Gnaphalium sandwicensium Gaud., Bot. Voy. Freyc. Uranie $466,(1826)=[1830]$.

G. sandwicensium Gaud. var. typicum Sherff forma canum Sherff, Am. Jour. Bot. 36: 504, 1949. 
G. sandwicensium Gaud. var. typicum Sherff forma olivaceum Sherff, Am. Jour. Bot. 36: 504, 1949.

162. Alcicomium Gaud., Bot. Voy. Freyc. Uranie 48, 1826. Platycerium Desv., Linn. Soc. Paris, Mém. 6(2): 213, 1827.

163. Gerbera (viridifolia var.?) Conrathii Thell. spec. vel var. nov., Naturf. Gesell. Zürich, Vierteljahrschr. 68: 454, 1923.

164. Trematosphaeriopsis Elenkin, Jard. Bot. St. Petersb., Bul. 136-146, 1901.

Trematosphaeris Clements, Gen. Fungi 35, 1909.

165. Aecidium Violae Schum., Enum. Pl. Saell. 2: 224, 1803. Uredo Violae Schum., Enum. Pl. Saell. 2: 233, 1803. Puccinia violae DC., Fl. Fr. 6: 62, 1815.

P. densa Diet. \& Holw. in Dietel, Hedwigia 36: 298, 1897.

Dicaeoma Violae (DC.) Ktze., Rev. Gen. Pl. 3(3): 471, 1898. Puccinia violae (Schum.) DC. ex Arthur, J. C., Man. Rusts U. S. \& Can. 311, 1934.

166. Fraxinus juglandifolia Lam., Encyc. Méth. Bot. 2: 548, $(1786)=[1788]$.

F. americana L. juglandifolia D. J. Browne, Trees Am. 398, 1846.

F. americana L. var. juglandifolia [Lam.] D. J. Browne ex Rehder, Bibliog. Cult. Trees \& Shrubs 557, 1949.

167. Arachis hypogaea L. var. microcarpa A. Chevalier, Rev. de Bot. Appl. et Agr. Trop. 9: 193, pl. 5, fig. 3; pl. 6, fig. 5, 1929.

A. hypogaea L. forma microcarpa (A. Chev.) Hermann, U. S. Dept. Agric., Monogr. 19: 14, 1954.

168. Lipotriche sensu Less., Syn. Gen. Composit. 231, 1832;

Linnaea 6: 510, 1831.

Lipotriche R. Br., Linn. Soc. Lond., Trans. 12: 118, 1817. Lipochaeta DC., Prodr. 5: 610, 1836. Microchaeta Nutt., Am. Phil. Soc., Trans. II, 7: 450-452, 1841.

Schizophyllum Nutt., Am. Phil. Soc., Trans. II, 7: 452-453, 1841.

See Sherff, Bishop Mus., Bul. 135: 24, 1935. Silva, Univ. Calif., Publ. Bot. 25: 281, 1952. 
169. Nicandra physaloides (L.) Gaertn., Fruct. 2: 237-238, pl. 131, fig. 2, $(1802)=[1791]$. Atropa physalodes L., Sp. Pl. 181, 1753. Physalodes peruvianum Ktze., Rev. Gen. Pl. 452, 1891. Nicandra Physalodes (L.) Pers. ex Robins. \& Fern., in Gray's Man., ed. 7, 716, 1908.

Physalodes Boehm., ed. of Ludw. Def. Gen. Pl. 41-42, 1760.

Physaloides Moench, Meth. Pl. Hort. Bot. Marburg. 473, 1794.

Pentagonia physalodes (L.) Hiern, Cat. Welw. Afr. Pl. 1: $752,1898$.

Physalodes physalodes (L.) Britton, Torrey Bot. Club, Mem. 5: 287, 1894.

170. Viguier, R. ( + ), issued by Meslin, R., Les Legumineuses de Madagascar. Archives de Botanique 6: 1-823, 1944. There were printed 500 copies, of which 498 were destroyed while still in the printing house in the Battle of Paris, 1944. Two copies now exist, one with M. Meslin, one in the Museum National d'Histoire Naturelle, Paris. The book contains numerous new species.

171. Teucrium cubense Jacq. ssp. depressum (Small) McClintock \& Epling, Brittonia 5: 505, 1946, Sept. 6.

T. depressum Small, New York Bot. Gard., Bul. 1: 288, 1899.

T. cubense Jacq. var. densum Jepson, Man. Fl. Pl. Calif. 861, 1925, Sept. 23 ?

See L. C. Wheeler, Am. Jour. Bot. 29: 19s, 1942, Dec.

172. Angervilla Neck., Elem. Bot. 1: 351, 1790. Matourea Aublet, Hist. Pl. Guiane 2: 641-643, 1775; 4: pl. $259,1775$.

Stemodia L., Syst. Veg., ed. 10, 1,118, 1759.

173. Pandanus aimiritkensis Martelli in Kanehira, Fl. Micrones. 60, fig. 1, 1933; Kyushu Imp. Univ., Dept. Agric., Jour. 4(6): 259, 1935, nom. seminud.; Bot. Mag. Tokyo 48: 125, fig. 7, 1934.

174. Fagaster Dombeyi (Mirb.) Spach, Hist. Nat. Vég. Phan. 11: 142, 1842. 
175. Triticum strictum Detharding, Consp. Pl. Magn. Megap. 11, 1828.

Agropymum strictum (Dethard.) Reichb., Fl. Germ. Excurs. $21,1830$.

Triticum elymogenes Arndt, Flora 42: 215, 1859.

Triticum junceum $\times$ Hordeum (Elymus) arenarium Marsson,

Fl. Neuvorp. u. Rügen 598, 1869.

Triticum junceum $\times$ Elymus arenarius Nyman ex Aschers. \& Graebn., Syn. Mitteleur. Fl. 2(1): 748, 1902. $\times$ Tritordeum strictum Asch. \& Graebn., Syn. Mitteleur. Fl.

2: 748, 1902.

Agropymm junceum $\times$ Elymus arenarius G. Camus, Mus.

Natl. Hist. Nat. Paris, Bul. 6: 538, 1927.

$\times$ Agroelymus strictus (Reichb.) G. Camus in A. Camus,

Mus. Natl. Hist. Nat. Paris, Bul. 6: 538, 1927. $\times$ Agroelymus strictus (Dethard.) G. Camus ex Rousseau,

Jard. Bot. Montreal, Mem. 29: 14, 1952.

176. Agropsammelymus Lepage, Nat. Canad. 80: 196, 1953.

177. $\times$ Agroelymus Adamsii Rousseau n.m. jamesensis Lepage,

Nat. Canad. 80: 197, 1953.

$\times$ Agroelymus jamesensis Lepage, Nat. Canad. 79: 245-246, 1952.

178. Acer sacchamum Marsh cl. 'Newton Sentry' B. Harkness,

Baileya 2: 99-101, fig. 31B, 1954.

A. saccharinum columnare Temple, Temple's Cat. 1887.

179. Equisetum sylvaticum L. var. pauciramosum Milde forma neoserotinum Victorin, Univ. Montréal. Lab. Bot.,

Contr. 9: 120, 1927.

See Boivin, Can. Field Nat. 63: 75, 1949.

180. Juggerlandia mucida (Schulzer) McGinty ex Lloyd, Mycol.

Writ. 7: 1,230-1,231, pl. 259, fig. 2,570, 1923.

J. mucida (Schulzer) Lloyd teste Stevenson \& Cash, Lloyd

Libr. \& Mus., Bul. 35 (Mycol., Ser.8): 26, 1936.

181. Eragrostis perplexa L. H. Harvey, Univ. Microfilms, Publ.

967: 1948; Torrey Bot. Club, Bul. 81: 409-410, 1954.

182. $\times$ T sugo-Piceo-T suga Jeffreyi (Henry) Campo-Duplan \&

Gaussen, Lab. For. Toulouse, Trav. I, 4(24): 11, 1948. Tsuga Jeffreyi (Henry) Henry, Roy. Irish Acad., Proc.

35B(4): 55, 1919. 
T suga Pattoniana Engelm. var. Jeffreyi Henry in Elwes \& Henry, Trees Gr. Brit. \& Irel. 2: 231, 1907.

183. Culhamia Forsk., Fl. Aegypt.-Arab. 96, 1775.

184. Juglandicarya integrifoliolata (Kuang) Hu, Paleobot. 1: 264, 1952.

Carya sinensis Dode, Soc. Dendrol. France, Bul. 24: 61, 1912.

Annamocarya indochinensis Cheval., Intern. Bot. Appl., Rev. 21: 504, 1941.

Juglans indochinensis Cheval., Intern. Bot. Appl., Rev. 21:

504, 1941.

Rhamphocarya integrifoliolata Kuang, Icon. Flor. Sinicae 1:

1-2, pl. 1, 1941.

Annamocarya sinensis (Dode) Leroy, Intern. Bot. Appl.,

Rev. 30: 426, 1950; Acad. Sci. Paris, Compt. Rend.

232: 432-434, 1,007-1,009, 1951.

See Scott, Am. Jour. Bot. 40: 666-669, 1953.

185. Bryum argenteum L., Sp. PI. 1,120, 1753.

$B$. argenteum L. ex Hedw., Sp. Musc. 181, 1801.

186. Fucus Theophrastus, Peri Phuton Historia 4: 6-2; 4: 6-9;

Inquiry into Plants, ed. A. Hort 1: 328, 334, 1916.

Fucus Tourn., Inst. Rei Herb. 1: 565-569; 3: pl. 334-336,

1700.

Fucus S. F. Gray, Nat. Arr. Brit. Pl. 1: 389, 1821.

Muscus marinus C. Bauhin, Pinax 363-364, 1671.

187. Dendrophtho ̈̈ Mart., Flora 13(1): 109, 1830.

Dendrophtoe pentandra (L.) Miq., Fl. Ind. Bat. 1(1): 818,

$(1855)=[1858]$.

Loranthus pentandmes L., Mant. 1: 63, 1767.

$L$. pentandrus sensu Heyne ex R. \& S., in Syst. Veg. of

Linnaeus, ed. nov. by R. \& S., 7: 112, 1829.

L. longifloms Desr. in Lam., Encyc. Méth. Bot. 3: 598,

$(1789)=[1791]$.

188. Varengevillea Baill., Hist. des PI. 11: 116, 1892.

V. hispidissima (Seem.) Baill., Hist. des Pl. 11: 116, 1892.

Colea hispidissima Seem., Linn. Soc. Lond., Trans. 23: 9,

1860.

See Perrier de la Bathie, Not. Syst. 13: 290-291, 1948. Moldenke, Phytologia 5: 149, 1955. 


\section{CHAPTER 3}

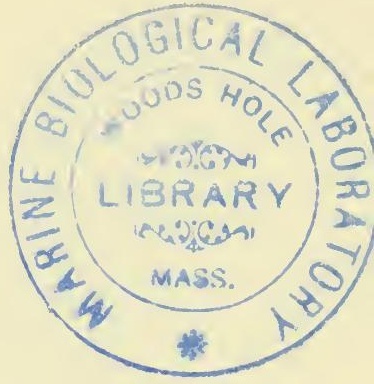

200. Aleurodiscus Rab. ex Cke., Grevillea 3: 137, 1875.

Aleurodiscus Rab. ex Schroet. in Cohn, Crypt.-Fl. Schles.

1: 429, 1888.

Cyphella Fr., Syst. Mycol. 2: 201, 1822.

See Donk, Bot. Gard. Buitenzorg, Bul. III, 17: 156, 159-

160, 1941; 18: 88-89, 159, 1949; Reinwardtia 1:

206-208, 1951.

Rogers \& Jackson, Farlowia 1: 269, 1944.

Rogers, Farlowia 3: 433, 1949; 4: 15, 1950.

201. Erythrophyllum Loeske, Hedwigia 47: 175, 1908.

Erythrophyllum Agardh, Lunds Univ. Ars-Skr., Afdeln. III

Math. \& Naturvetsk. 8(6): 10-12, 1871.

Erythrobarbula Steere, Bryologist 54: 191, 1951.

202. Penecillium frequetans Series, Raper, Thom \& Fennel, Man.

Penecillia 170, 1949.

P. frequetans Westling, Arkiv för Botanik 11: 133, fig. 39,

78, 1912.

203. Spongodendron Zanardini, Nuovo Giorn. Bot. Ital. 10: 3738, 1878.

Spongocladia Areschoug, Öfvers K. Vetensk.-Akad.,

Förhandl. 10: 201-209, 1853.

Cladophoropsis Boergesen, Overs, K. Danske Vidensk.

Selsk., Förhandl. 1905: 259-291, 1905.

See Papenfuss, Pacif. Sci. 4: 208-213, 1950.

Silva, Univ. Calif., Publ. Bot. 25: 271, 1952.

Taxon 3: 234, 1954; 4: 118, 1955.

204. Peridinium depressum Bailey, Smithsonian Inst., Contr.

Know. 7(3): 12ळ13, pl., fig. 33-38, 1855.

Ceratium divergens Claparède \& Lachman, Inst. Natl.

Génév., Mem. 1859-1860, 7: 71, pl. 13, fig. 23, 1861,

non Ehrenberg (1840). 
P. divergens Ehrenb. $\gamma$ reniforme Ehrenb., Akad. Wissensch. Berlin, Ber. 240, 1854.

$P$. depressa Ostenfeld in Knudsen \& Ostenf., Iagttagelser over Overfladevandets Temperatur, Wandel. 60, fig. 1, 1900.

P. marinum Lindamann, Bot. Arch. 9: 98-99, fig. 7-12, 1925.

See Graham, Carnegie Inst. Wash., Publ. 542; 18-21, 1942.

205. Roscheria H. Wendl. in Baker, Fl. Maurit. 386-387, 1877. Roscheria Wendl. in Papenfuss, Madroño 11: 29, 1951; repr. p. 4, 1951.

Roschera Sonder in von der Decken, Reisen in Ost-Afrika in 1859-1865, 3(3): 81, pl. 1, fig. 5-11, 1879.

Tolypiocladia Schmitz in Engler \& Prantl, Natürl.

Pflanzenfam. 1(2): 441-442, 1897; Engl. Bot. Jahrb. 21: $160,1895$.

See Silva, Univ. Calif., Publ. Bot. 25: 308, 1952.

206. Tricholoma quercifolia Murrill, Lloydia 12: 67, 1949.

T. subfulvidiscum Murrill, Lloydia 12: 67, 1949.

207. Halymenia Durvillaei Bory var. denudata Weber-van Bosse, Siboga Exped., Monogr. 59b: 236, 1921.

208. Lycopodium Hamiltonii Spreng., in Syst. Veg. of Linnaeus, ed. 16 by Spreng. 5: 429, 1829; 4(1): 20, 1827; 4(1): $12,1827$.

209. Ahnfeltia concinna J. Agardh, Ofvers K. Svensk. Vet. Akad., Förhandl. 1847(1): 12, 1847.

A. Gigartinoides J. Agardh, Ofvers. K. Svensk. Vet. Akad., Förhandl. 1847(1): 12, 1847.

A. concinna J. Agardh in Setchell \& Gardner, Univ. Calif.

Publ. Bot. 1: 305, 1903.

A. Gigartinoides J. Agardh in G. M. Smith, Marine Algae Monterey Penin. Cal if. 272, 1944.

210. Peziza amorpha Pers., Mycol. Eur. 1: 269, 1822.

Thelephora amorpha (Pers.) Fries, Elenchus Fung. 1: 183, 1828.

Corticium amorphum (Pers.) Fries, Epicr. Syst. Mycol. 559, 1838. See Saccardo, Syll. Fung. 6: 606, 1888. 
Cyphella amorpha (Pers.) Quél., Ench. Fung. 215, 1886. Aleurodiscus amorphus (Pers.) Rabenh., Fungi Eur. Exs.

1,824, 1874; Hedwigia 13: 184, 1874.

A. grantii Lloyd, Mycol. Writ. 6: 9227, fig. 1,668, 1920.

Nodularia balsamicola Peck, New York State Mus., Rept.

24: 96, pl. 4, fig. 23-26, 1872.

See Taxon 3: 234, 1954; 4: 118, 1955.

211. Trichogloea Herveyi Taylor, Hydrobiologia 3: 119-121, 1951.

T. Herveyi Setchell in Howe in Britton, Fl. Bermuda 511, 1918.

212. Agardhiella Schmitz, Flora 7: 441, 1889.

213. Microsorium punctatum (L.) Copel., Bishop Mus., Bul. 93:

73, 1932.

Polypodium punctatum (L.) Sw., Schrad. Jour. 1800(2): 21, 1801.

Acrostichum punctatum L., Sp. Pl., ed. 2, 2: 1,524, 1763.

214. Marsilea L., Gen. Pl., ed. 5, 485, 1754; Sp. Pl. 1,099, 1753.

215. Goniolithon Foslie, Kgl. Norske Vidensk. Selsk., Skrift. 2:

$5,1898$.

See Setchell \& Mason, Natl. Acad. Sci., Proc. 29: 87-97, 1943.

216. Albugo Pers. ex S. F. Gray, Nat. Arr. Brit. Pl. 1: 540, 1821. Cystopus Lév., Ann. Sci. Nat. Bot. III, 8: 371, 1847. Cystopus De Bary, Ann. Sci. Nat. Bot. IV, 20: 31, 1863.

See Ramsbottom, Jour. Bot. Brit. For. 54: 77, 1916; Brit. Mycol. Soc., Trans. 18: 315, 1934.

Wakefield, Bothalia 2: 242-246, 1927.

Fitzpatrick, The Lower Fungi: Phycomycetes 192, 1930.

Weatherby, Kew Bul. Misc. Inf. 421-422, 1935.

Ainsworth \& Bisby, Dict. Fungi 80, 1943; ed. 3, 91, 1950.

Rogers, Mycologia 40: 241-254, 1948; 42: 325-330, 1950.

217. Struthiopteris pensylvanica Willd., Sp. Pl. of L., ed. 4 by Willd. 5: 289, 1810. Onoclea Struthopteris (L.) Hoffm., Deutschl. Fl. 3: 11, 1795. Matteucia Struthiopteris (L.) Todaro, Syn. Pl. Acot. Vasc.

Sicilia 30, 1866. 
Osmunda Struthiopteris L., Sp. Pl. 1,066, 1753.

Pteretis pensilvanica (Willd.) Fernald, Rhodora 47: 123, 1945.

Matteucia Struthiopteris (L.) Todaro var. pensylvanica (Willd.) Morton, Am. Fern. Jour. 40: 247, 1950.

218. Fumago Pers., Mycol. Eur. 1: 9, 1822.

See Berk. \& Mont., Hort. Soc. Lond., Jour. 4: 244-245, 1849.

Woronich, Ann. Mycol. 24: 261-264, 1926.

Bisby, Commonw. Mycol. Inst., Mimeogr. Publ. 6: 11, 1949.

Rogers, Mycologia 40: 635-637, 1948; Farlowia 4: 17-18, 37-38, 1950.

219. Lycopodium inundatum L. var. appressum Chapm., Bot.

Gaz. 3: 20, 1878.

L. alopecuroides L. var. adpressum Chapm. emend., Fl. So.

U. S., ed. 2, 671, 1883; ed. 3, 638, 1897.

See Fernald, Rhodora 42: 405, 1940.

220. Martensia Hering, Ann. and Mag. Nat. Hist. 8: 92, 1842. Hemitrema R. Br. ex Endl., Mant. Bot. sistens Gen. Pl., Suppl. 3: 50, 1843.

Mesotrema J. Agardh, Övers K. Svenske Vetensk.-Akad.,

Förhandl. 11: 110, 1854.

Capraella De Toni f., Not. Nomencl. Alg. 7: 3, 1936.

Martensia Giseke, Linn. Praelec. Ord. Nat. Pl., ed. Giseke 199, 207, 249, 1792.

Mertensia Roth, Catalect. Bot. 1: 34, 1797; HBK., Nov.

Gen. Sp. 2: 30-32, pl. 103, 1817.

See Papenfuss, Natl. Acad. Sci., Proc. 28: 448, 1942;

Madroño 10: 182, 1950; Doty, Taxon 3: 229, 1954.

Taxon 3: 234, 1954; 4: 118, 1955.

221. Elizabethia miniata Trevisan, Gazzerta Uffiziale di

Venezia, anno 1855(53): 3, 1855, marzo 6.

See de Toni, Archvio Botanico 14(3-4): 9-10, 1938.

Silva, Univ. Calif., Publ. Bot. 25: 301, 1952.

222. Aspidium scabriusculum Davenp., Bot.Gaz. 21: 255-256, 1896.

Dryopteris scabriuscula Davenp., Bot. Gaz. 21: 256, 1896. 
Nephrodium scabriusculum Davenp., Bot. Gaz. 21: 256, 1896.

Lastrea scabriuscula Davenp., Bot. Gaz. 21: 256, 1896.

223. Cibotium NEALIAE Degener in Degener \& Hatheway, Fl.

Haw. 5: fam. 12, 8/15/51.

C. glaucum var. NEALIAE Hatheway in Degener \& Hatheway, Fl. Haw. 5: fam. 12, 8/15/51.

224. Flammula (Fr.) Kummer, Führer Pilzk. 22, 1871. Flammula (DC.) Fourr., Soc. Linn. Lyon, Ann. II, 16: 324, 1868.

Gymnopilus Karst., Känned. Finl. Nat. Folk, Bidr. 32: xxi, 400, 1879.

See Dodoens, Stirp. Hist. Pempt. 399-400, 1553.

De Candolle, Reg. Veg. Syst. Nat. 1: 133, (1818)= [1817].

Wakefield, Brit. Mycol. Soc., Trans. 23: 227, 1939. Donk, Bot. Gard. Buitenzorg, Bul. III, 17: 157, 166168, 1941; 18: 95-96, 1949; 18: 328, 332, 1949.

Singer \& Smith, Mycologia 38: 263, 291, 1946.

Rogers, Farlowia 3: 443, 1949; 4: 25, 1950.

225. Lycopodium annotinum L. forma spica omacio sessile

Luerssen, Nordostdeutsche Waldfl., 1880.

L. annotinum L. lusus proliferum Aschers. \& Graebn., Syn.

Mitteleurop. F1. 1: 152, 1896.

See Nessel, Die Bärlappgewächse 278, 1939.

226. Uromyces appendiculatus (Pers.) Link, Ges. Nat. Freunde Berlin, Mag. 7: 28, 1816.

Uredo appendiculata Pers., Ann. Bot. Usteri 15: 16-17,

1795; Syn. Meth. Fung. 221-222, 1801.

Uredo Pamparum Speg., Soc. Ci. Argent., Anal. 9: 173, 1880. Uredo rufa (Bon.) Speg., Soc. Ci. Argent., Anal. 17: 124, 1884.

See Jackson, Mycologia 23: 351, 1931.

227. Zanardinula Kylinii Doty, Farlowia 3: 173, 1947. Prionitis linearis Kylin, Univ. Lund., Arsk. N. F. 37(2):

12-13, pl. 4, fig. 11, 1941.

Z. linearis (Kylin) Papenfuss, Farlowia 1: 342, 1944. $P$. linearis Kylin in Smith, Marine Algae Monterey Penin. 245, pl. 56, fig. 21, 1944. 
Prionitis Agardh, Sp. Gen. Ord. Alg. 2: 185-191, 1851. $P$. lanceolata Harvey sensu Collins, Holden \& Setchell in Collins et al., Phytotheca Bor. Am. fasc. 4: No. 199b, 1896.

Gelidium ? lanceolatum Harvey in Hook. \& Arn., Bot. Beechey Voy. 164, 409, 1841.

P. lanceolata (Harvey) Harvey, Smithsonian Inst., Contr. 5: 197, pl. 27, fig. A, 1852.

Z. lanceolata (Harvey) de Toni, Noterelle di Nomencl. Alg. 7: sixth page, unnumbered, 1936.

See Taxon 3: 234, 1954; 4: 118, 1955.

228. Andreaea Ehrh., Hannov. Mag. 1778(or 16): 1,601-1,603, 1779. Andreaeaceae Lindb., Utkast 36, 1878. Andreaeales Ruhland in Engler, Natürlichen Pflanzenfam. 1(3): 262, 1909; ed. 2, 10: 126, 1924.

229. Zonaria Roussel, Flore du Calvados, ed. 2, 99-100, 1806. Zonaria C. Agardh, Syn. Algarum Scand., p. XX, 1817. Zonaria C. Agardh sensu Tandy, Brittonia 6: 37, 1947. Zonaria C. Agardh sensu Papenfuss, Madroño 9: 9, 1947. Gymnosomes J. Agardh, Analecta Algol., Contin. 1., Soc. Physiogr. Lund., Act. 29: 9-12, 1894.

Gymnosorus Trevisan, Saggia di monograf. alghe coccothalle 108, 1848.

Orthosomes Trevisan, Linnaea 22: 457-461, 1849. Pocockiella Papenfuss, Am. Jour. Bot. 30: 463-468, 1948.

See Silva, Univ. Ca lif., Publ. Bot. 25: 272-277, 1952;

Taxon 3: 234, 1954; 4: 118, 1955.

230. Equisetum ramosis simum Desf. Race III.-E. campanulatum Poir. $\beta$ scabrum Hy, S.-var. Renati (Hy, 1. c., pro var.) Nob., Rouy. Flore de France 14: 507, 1913. E. ramosissimum Desf., Fl. Atlant. 2: 398-399, anno sexto $(1800)=[1799]$; see Stearn, Soc. Bibliog. Nat. Hist., Jour. 1: 147-148, 1938.

E. campanulatum Poir. in Lam., Dict. 5: 613, 1804. E. ramosissimum Desf. 1 scabrum Milde, Höheren Sporenpfl. Deutschl. \& Schweiz 118, 1865.

231. Urostachys erubescens (Brack.) Herter ex Nessel, Die Bärlappgewächse 61, 1939. 
Lycopodium erubescens Brack., U. S. Expl. Exped. Bot. 16: 320, pl. 45, fig. 1, 1854.

232. Dumortiera hirsuta ( $\mathrm{Sw}_{\text {. }}$ ) Reinw. Bl. \& Nees var. nepalensis . (Tayl.) Frye \& Clark, Hepat. N. Am. 93, 1937.

Hygropyla nepalensis Tayl., Linn. Soc. Lond., Trans. 17:

$392,(1835)=[1837]$.

Dumortiera nepalensis (Tayl.) Nees, Naturg. Eur. Leberm.

4: 169, 1838.

D. hirsuta $\alpha$ latior Gottsche, Lind. \& Nees, Syn. Hepat. 544, 1844.

233. Dryopteris gongylodes (Schk.) Ktze., Rev. Gen. Pl. 2: 811, 1891.

Aspidium goggilodus Schk., Vier und Zwanzig. Kl. Linn.

Krypt. Gewachse 1: 193; 2: pl. 33c, 1809.

Cyclosome gongylodes (Schk.) Link, Hort. Reg. Bot. Berol.

2: $128,1833$.

Dryopteris goggilodus (Schk.) Fosb., Bishop Mus., Occ.

Pap. 16: 337-338, 1942.

See Skottsberg, Nat. Hist. Juan Fernandez \& Easter I., 2: 768, 1951.

234. Polystichum aristatum (Forst. f.) Presl var. Voniifolium

(Wall.) Farw., Am. Midl. Nat. 12: 261, 1931.

Aspidium aristatum (Forst.f.) Sw. $\beta$ A. Coniifolium (Wall.)

Hook. \& Baker, Syn. Fil. 255-256, 1874.

A. Coniifolium Wall., Cat. (Num. List Pl. E. Ind.) No. 341, 1828.

A. coniifolium Presl, Del. Prag. 1: 175, 1822.

235. Ectocarpus Duchassaingianus Grunow, ed. Fenzl, Reise der

Österreich. Fregatte Novara um die Erde, Bot. Theil. 1: 45, pl. 4, fig. 1, 1870.

See Silva, Univ. Calif., Publ. Bot. 25: 256, 1952.

236. Nectria cytisporina Ellis \& Everh., Erythea 1: 197, 1893.

237. Anthoceros Micheli, Nova Genera P1. 10, 1729. Anthoceros [Micheli] L., Sp. Pl. 1,139, 1753. Anthocerus L. ex Dillen., Hist. Musc. 11: 1768. Ceranthus L., Fund. Bot. of Linnaeus, ed. Gilibert, 3: 466,

1787 (Gilibert, Syst. Pl. Eur. 7: 466, 1787). Corypta Necker, Elem. Bot. 344, 1790.

Carpoceros Du Mortier, Comment. Bot. 76, 1822. 
Carpoceras DC., Mus. Paris, Mém. 7: 234, 1821.

Anthoceras L. ex Brongn. \& Dec., Ann. Sci. Nat. Bot. V, 20:

325, 1874.

Aspiromitus Steph., Sp. Hepat. 5: 957, 1916.

See Proskauer, Ann. Bot. n.s., 12: 262, 1948.

238. Pauahia sideroxyli Stevens, Bishop Mus., Bul. 19: 17-18, fig. 2, 1925.

239. Langermannia Rostk. in Sturm, Deutschl. Fl. 3(5): 3, 23,

1844.

Calvatia Fr., Summa Veg. Scand. 2: 442, 1849.

See Perdeck, Blumea 6: 486, 1950.

240. Raciborskiomyces Siemaszko, Soc. Bot. Pol., Act. 2: 270, 1925.

Chatyllis Clements in Clements \& Shear, Gen. Fungi 253, 1931.

241. Sphaeria picea Pers., Icon. Descr. Fung. 2: 40, pl. 10, fig. 7-8, 1800.

S. picea Pers. ex Fr., Syst. Mycol. 2(2): 431, 1823.

Diaporthe Euporthe picea (Pers.) Sacc., Syll. Fung. 1: 648, 1882.

242. Mycosphaerella elymifoliae Munk, Dansk Bot. Ark. 14(8): 2, 1952.

243. Cladophora Aegagropila Sibogae Rbld. ex Weber, Siboga

Exped., Monogr. 59a: 81-82, fig. 19, 1913.

C. (Aegagr.) Sibogae Rbld., Nuova Notarisia 16: 146, 1905. See Taxon 3: 234, 1954; 4: 118, 1955.

244. Euzodiomyces Thaxt., Am. Acad. Arts Sci., Proc. 35: 449, 1900.

245. Peniophora ludoviciana Burt, Mo. Bot. Gard., Ann. 12: 244, 1925.

246. Puccinia Pers., Syn. Meth. Fung. xv, index, 1801. Pvccinia Pers., Syn. Meth. Fung. 225-230, 1801.

247. Pellicularia Cke., Grevillea 4: 116, 1876.

248. Fimbriaria Bolanderi Austin, Acad. Nat. Sci. Phila., Proc. 1869: $230,(1869)=[1870]$.

Asterella Bolanderi (Austin) Underw., Bot. Gaz. 20: 61, 1895.

See Silva, Univ. Calif., Publ. Bot. 25: 269, 1952. 
249. Leptodontium humillimum Broth., Rijks Herb. Leiden, Meded. 14: 17, fig. 1, 1912.

250. Epichloe (Fr.) Tul., Sel. Fung. Carpol. 3: 24, 1865. Epichloe Fr., Summa Veg. Scand. 381, 1849.

Epichlö̈ (Fr.) Tul. ex Atkins., Torr. Bot. Club, Bul. 21: $224,1894$.

251. Cladonia subrangiformis Sandst., Naturw. Ver. Bremen, Abhandl. 25: 165, 1922.

C. subrangiformis Scriba ex Sandst., in Rabenh., Krypt. Fl.

Deutschl. Oesterr. Schw. $9\left(4^{2}\right): 231,1931$.

C. furcata (Huds.) Schrad. C. subrangiformis (Sandst.) Des

Abbayes, Soc. Sci. Bretagne, Bul. 14: 154, 1937.

See Evans, Rhodora 56: 267, 1954.

252. Agaricus Persoonii Fr., Syst. Mycol. 1: 27, 1821. A. Persooniana Phillips \& Plowr., Grevillea 10: 66, 1881.

253. Boudiera Cke., Grevillea 6: 76, 1877.

254. Brongiart, Essai Class Champ. 1825.

255. Gaultheria Kalm in L., Gen. Pl., ed. 5, 187, 1754. Gautiera Raf., Med. Fl. 1: 202, 1828. Gautieria Vitt., Mon. Tuber. 25, 1831. Gualteria Raf., Fl. Tellur. 1: 18, (1836) = [1837]. Gaultiera Raf., Fl. Tellur. 1: 18, $(1836)=[1837]$.

See Rogers, Farlowia 4: 25-26, 1950.

256. Boletus Tulipiferae Schw., Naturf. Ges. Leipzig, Schr. 1: 99, 1822.

Irpex tulipifera (Schw.) Schw. ex Fries, Epicr. Syst. Myc. 523, 1838; Overh., Mo. Bot. Gard., Ann. 1: 152, 1914. Polyporus tulipiferus Schw. ex Overh., Wash. Univ., Stud. 3(1), No. 1: 29, pl. 3, fig. 11a-c, 1915.

257. Ravenelia Berk., Gard. Chron. No. 9: 132, fig. 1-2, 1853, Feb. 26.

258. Höhnelogaster Lohwag, Bot. Centralbl., Beih. 42(2): 299, 1926.

Hoehneliogaster Lohwag emend. E. Fisch., in Engler \&

Prantl, Natürl. Pflanzenfam., ed. 2, 7a: 23, 1933.

259. Helotium ? brassicaecolum (Schw.) Sacc., Syll. Fung. 8: 226, 1889. 
Sarea brassicaecola Schw., Am. Phil. Soc., Trans. II, 4: $178,1832$.

260. Agaricales Clements ex Singer, sensu strictiore, Lilloa 22: $126,(19491=[1951]$.

Agaricales Clements, Gen. Fungi 102, 1909; Rea, Brit. Bas. xi, 1922.

261. Lenormandia coronata Lindauer \& Setch. in Lindauer, Algae Nova-Zelandicae Exsiccatae 74: 1940; Lindauer \& Setch., R. S. New Zealand, Trans. 76(1): 66-68, pl. 1, 1946.

See Taxon 3: 234, 1954; 4: 118, 1955.

262. Enteromorpha compressa constricta J. Agardh in M. A.

Howe, Torrey Bot. Club, Bul. 57: 605, 1931.

E. compressa (L.) Grev. b. constricta J. Agardh, Lunds

Univ., Arsskrift 19: 138, 1882-1883.

See Taxon 3: 234, 1954; 4: 118, 1955.

263. Chlorococcum E. M. Fries, Syst. Mycol. 1: XXII, 1821; 2:

$272,1825$.

Tremella botryoides (L.) Schreber, Spicil. Fl. Lips. 141,

1771.

Byssus botryoides L., Sp. Pl. 1,169, 1753.

Nostoc Botryoides (L.) Agardh, Syn. Alg. Scand. 135, 1817. Protococcus botryoides (Kuetz.) Kirchn., in Cohn, Kryptog.

F1. Schlesien 2(1): 103, 1878.

See Silva, P. C. \& Starr, R. C., Svensk Bot. Tidskr. 47: 234-247, 1953.

264. Hymenophyllum polyanthum Hook. in Nightingale, Thos.,

Oceanic Sketches 132, 1835.

Trichomanes polyanthos (Hook.) Hook., Sp. Fil. 1: 138,

1846; Hooker's Icones 8: text and pl. 703, 1848.

See Copel., Philipp. Jnur. Sci. 51: 230, 1933;

Ballard, Pacif. Sci. 10: 269-270, 1956.

265. Alsophila? polypodioides Hook. in Thomas Nightingale,

Oceanic Sketches 131, 1835.

Dryopteris ornata (Wall.) C. Chr., Ind. Fil. 281, 1905.

Polypodium ornatum Wall., Cat. (Num. List Pl. E. Ind.), No. $327,1829$. 
P. ornatum Wall. ex Beddome, Ferns S. India 56, pl. 171, 1863-1865.

See C. Chr., Bishop Mus., Bul. 177: 94, 1943.

266. F. Hildeb. =Hildebrand, Friedrich Hermann Gustav (1835-

1915). Determine abbreviations for: Hildebrandt, Johann Maria (1847-1881); Hillebrand, William (1821-1886);

Hillebrandt, Franz (1805-1860); Hill, John (1716-1775);

Hill, Albert Frederick (1889- ); Hill, Sir Arthur

William (1875-1941).

267. Polygonum convolvuloides Bruger forma intermedium

Lawalrée, Bruxelles Jard. Bot. Etat, Bul. 22(3-4):

211-212, fig. 26A, 1952.

$P$. convolvuloides Bruger forma pterocarpum Lawalrée,

Bruxelles Jard. Bot. Etat, Bul. 22(3-4): 212-213, fig. 26B, 1952.

268. Pseudendoclonium arctica Prescott, Am. Midl. Nat. 50: 467$468,(1953)=[1954]$.

269. Fucus scoparius Strøm, Physisk og Oeconomisk Beskr.

Fogderiet Sфndmфr Bergens Norge 1: 93-94, 1762;

Beskrivelse Norske Søe-Vaexter 250-252, pl. f, fig. 1,

1770.

Laminaria scoparia (Strøm) Du Rietz, Bot. Notis 1920: 42, 1920.

Fucus hyperboreus Gunnerus, Fl. Norveg. 1: 34-35, pl. 3, 1766.

Laminaria hyperborea (Gunn.) Foslie, Christiania Vidensk.-

Selsk., Forhandl. 14: 41-54, 1884; repr. as Ueber

Laminar. Norveg. 41-54, 1884.

Fucus digitatus L., Mantissa 134, (1771) = [1767].

Fucus digitatus Huds., Fl. Angl. 474, 1762.

Laminaria digitata (Huds.) Lamx., Nat. Hist. Mus. Paris,

Ann. 20: 42, 1813; repr. as Essai Gen. Thalassiophytes non Articulées 42, 1813.

Fucus scoparius Huds., Fl. Angl. 471, 1762.

See Du Rietz, Svensk Bot. Tidskr. 47: 16-23, 1953;

Silva, Univ. Calif., Publ. Bot. 25: 260, 1952.

270. Scyphofilix trrigosa (Thunb.) Farw. var. hirta (Kaulf.) Farw., Am. Midl. Nat. 12: 263, 1931. 
Trichomanes strigosum Thunb., Fl. Jap. 339-340, 1784. Davallia hirta Kaulf., Enum. Fil. 223, 1824.

Scyphofilix Thouars, Gen. Nov. Madag. 1, 1806; Roemer, Collect. Bot. 195, 1809.

271. Lecanora raroia Herre, Bryologist 56: 279-280, 1953.

272. Verrubotrytis Buchwald, Roy. Vet. \& Agric. Coll. Copenhagen, Dept. Pl. Path., Contr. 32: 146, 148, 1949.

273. Sphaerobotryotinia Buchwald, Roy. Vet. \& Agric. Coll. Copenhagen, Dept. Pl. Path., Contr. 32: 137, 1949.

274. Streptomyces albo-niger Hesseltine, J. N. Porter, Deduck, Hauck, Bohonos \& J. H. Williams, Mycologia 46: 19, 1954.

275. Verticilliodochium tubercularioides (Speg.) Bubàk, Ann. Mycol. 12: 220, pl. 8, 1914. Verticillis tuberculis (Speg.) Bubàk ex Clements \& Shear, Gen. Fungi 220, 401, 1931. Verticillium tubercularioides Speg., Fungi Argentini

Pugillus 4: No. 329, 1882; Soc. Ci. Argent. 13: 25, 1882.

276. Aecidium Pers. in Gmel., Syst. Nat. of Linnaeus, ed. 13 by Gmel., 2(2): 1,472, 1791. Oecidium Lév., in D’Orbigny, Dict. 1: 137, 1861.

See Arthur, J. C., Man. Rusts U. S. \& Can. 380, 1934.

277. Geminispora Pat., Soc. Mycol., Bul. 9: 151, 1893. Diplosporis Clements, Gen. Fungi 27, 1909.

278. Zygnema insigne (Hass.) Kütz. var. confusospora Habeeb, Rhodora 56: 42, 1954.

See Silva, Univ. Calif., Publ. Bot. 25: 253, 1952.

279. Bulgaria Fries, Syst. Myc. 2(1): 166, (1823) $=[1822]$. B. globosa Fries, Syst. Myc. 2(1): 166-167, (1823) =[1822]. B. inquinans (Pers.) Fries, Syst. Myc. 2(1): 167, (1823)= [1822].

Phaeobulgaria inquinans (Fr.) Seav., Mycologia 24: 253, 1932.

P. inquinans (Fr.) Nannf., Reg. Soc. Sci. Upsala, Nova Acta, IV, 8(2): 311, 1932. 
Bulgaria Fries ex Kobayashi, Jour. Jap. Bot. 13: 510-520, 1937.

See Seaver, N. Am. Cup Fungi (Inoperculates), 1951.

280. Sagenia plataphylla J. Sm., Hooker's Jour. Bot. 3: 410, 1841.

S. platyphylla J. Sm. emend., Ferns Brit. \& For., rev. ed., 145, 1879.

Aspidium platyphyllum (Willd.) Mett., Fil. Hort. Bot. Lips. 88, pl. 22, fig. 1-2, 1856.

A. platyphyllum Willd., Sp. Pl. of Linnaeus, ed. 4 by Willd. 5: 255, 1810.

A. platyphyllum (J. Sm.) Presl, Epim. 65, 1849.

281. Diplodina persicae Horn \& Hawthorne, Phytopath. 44: 134136, fig. 1, 1954.

282. Cordyceps Fr., Obs. Mycol. 2: 316, 1818.

Cordyceps Link, Handb. Gew. 3: 346, 1833.

Cordylia Fr., Obs. Mycol. 2: 316, 1818.

Cordylia Fr. ex Ficinus \& Schubert, Fl. Dresden, ed. 2, 2:

331, 1823.

Cordylia Pers., Syn. Pl. 2: 260, 1807.

Cordyceps Fr., Summ. Veg. Scand. 381, 1849.

Sphaeria ser. I Cordyliae Fr., Myk. 2: [33], 1823.

Sphaeria sect. I Periphericae trib. Cordyceps Fr., Syst.

Mycol. 2(2): 320, 323, 1823.

See Farlowia 3: 438, 1949;

Mycologia 46: 248-249, 1954.

283. Acharospora alboatra H. Magn., Bot. Staatssaml. München, Mitt. 9-10: 451, 1954.

284. Noteroclada Tayl. ex Hook \& W. Wilson, Hooker's London Jour. Bot. 3: 166, 1844.

Noteroclada Tayl. in Hook. f. \& Thos. Taylor, Hooker's

London Jour. Bot. 3: 477-478, 1844.

Androcryphia Nees in Gottsche, Lindenberg \& Nees, Syn.

Hepatic. 470-471, 1844.

See Taxon 3: 233, 1954.

Proskauer, Bryologist 58: 192-198, 1955.

285. Herbertus S. F. Gray, Nat. Arr. Brit. Pl. 684-685, 1821. Hervems S. F. Gray, Nat. Arr. Brit. Pl. 685, 1821. 
Herberta S. F. Gray emend. Lindb., Soc. Sci. Fenn., Acta 10: $516,1875$.

Pallavicinius S. F. Gray, Nat. Arr. Brit. Pl. 775, 1821.

Papa S. F. Gray, Nat. Arr. Brit. Pl. 686, 1821.

See Le Jolis, Cherbourg, Soc. Nat. Sci. Nat. Math., Mém. 29: 16, 1893.

Evans, Bryologist 40: 25-33, 1937.

286. Rhytisma astericolum Sacc., Syll. Fung. 8: 762, 1889.

287. Lithophyllum Hariot, sp. nov., Ministères Marine \& Inst.

Publ., Miss. Sci. Cap Horn, Bot. 5: 84, 1889.

288. Chrysophaeum Lewis \& Bryan, Am. Jour. Bot. 28: 343-348, 63 figs., 1941.

Chrysophaeum Taylor, Hydrobiologia 3: 122-130, 1951.

Chrysonephos Taylor, Torrey Bot. Club, Bul. 79: 79, 1952.

289. Rhizophidium difficile Canter, Brit. Mycol. Soc., Trans. 37:

119-121, fig. 4, 1954.

290. Albocmustum McG. ex Lloyd, Mycol. Writ. 7: 1,353-1,354, pl. 333, fig. 3,167-3,168, 1925.

Albocrustum LLoyd (as McGinty) teste Stevenson \& Cash, Lloyd Libr. \& Mus., Bul. 35 (Mycol., Ser. 8): 4, 1936.

291. Polypomes Fijii Lloyd, Mycol. Writ. 7: 1,112, pl. 194, fig. 2,077, 1922.

292. Globosopyreno Lloyd, Mycol. Writ. 7: 1,178, pl. 231, fig. 2,361-2,362, 1923.

293. Filicula bulbifera (L.) Farw., Am. Midl. Nat. 12: 251, 1931. Polypodium bulbiferum L., Sp. Pl. 1,091, 1753.

Cystopteris bulbifera (L.) Bernh., Schrad, Neu Jour. 1(2): $10,1806$.

294. Fomes arctostaphyli Long, New Mexico Chapter Phi Kappa

Phi, Papers 1: 2-3, 1917; Overholts, Polyporaceae

U. S., Alaska, Can. 60, 1953.

Boletus igniarius L., Sp. Pl. 1,176, 1753.

Polyporus igniarius L. ex Fries, Syst. Myc. 1: 375, 1821.

Fomes igniarius (L. ex Fries) Kickx., Fl. Crypt. Flandres

2: $237,1867$.

295. Itajahya Möll., Bras. Pilzblumen 79, 148, 1895. 
296. Trachyspora Fuckel, Bot. Zeit. 19: 250, 1861.

Trechispora Karst., Hedwigia 29: 147, 1890.

See Rogers, Mycologia 36: 75, 1944.

297. Hypnum torquatum Sw., Prodr. Ind. Occ. 142, 1788.

H. torquatum Hedw., Sp. Musc. 246, 1801.

Schlotheimia torquata (Sw. ex Hedw.) Brid., Bryol. Univ. 1:

323, 1826.

298. Gertmudia Herzog, Bibl. Bot. 87: 44, 1916.

299. Ceriosporopsis halima Linder in Barghoorn \& Linder,

Farlowia 1: 408-409, pl. 3, fig. 10-12, 1944.

Helicoma salinum Linder in Barghoorn \& Linder, Farlowia

1: 406-407, 1944. 


\section{CHAPTER 4}

300. Thelypteris spinulosa (O. F. Muell.) Nieuwl., var. dilatata (Hoffm.) St. John, Bot. Dept., State Coll. Wash., Contr. 2: $1,1925$.

Polypodium dilatatum Hoffm., Deutschl. Fl. 3: 7-8, 1795;

Fl. Germ. 2: 7-8, 1795.

Aspidium spinulosum (O. F. Muell.) Willd. var. dilatatum

(Hoffm.) Hook., Brit. Fl. 444, 1830.

Dryopteris spinulosa (O. F. Muell.) Ktze. var. dilatata

(Hoffm.) Underw., Nat. Ferns \& Allies, ed. 4, 116-117, 1893.

Nephrodium dilatatum (Hoffm.) Desv., Soc. Linn. Paris,

Mém. 6: 261, 1827; repr. as Prodr. 261, 1827.

301. Sabal louisiana (Darby) Bomhard, Wash. Acad. Sci., Jour. 25: 44, 1935.

Chamaerops louisiana Darby, Geogr. Descript. Louisiana 194, 205-206, 216, 1816.

Sabal Deeringiana Small, Torreya 26: 34, 1926.

S. ? adiantinum Raf., Fl. Ludov. 17, 1817.

302. Juglans pecan Marshall, Arbustr. Am. 69, 1785.

Hicoria Pecan (Marsh.) Britt., Torrey Bot. Club, Bul. 15:

282, 1888.

Hicorius Pecan (Marsh.) Sarg., Gard. \& For. 2: 460, 1889. Iuglans illinoinensis Wang., Nordam. Holz. 54-55, pl. 18 or fig. 43, 1787.

Carya illinoënsis (Wang.) K. Koch, Dendr. 1: 593-594, 1869.

Scoria Raf., Med. Repos. 11(ser. 2, 5:): 352, 1808.

Hickorius Raf., Fl. Ludov. 109, 1817.

Carya Nutt., Gen. Am. Pl. 2: 220-222, 1818.

See Sargent, Bot. Gaz. 66: 229-258, 1918.

Rehder, Jour. Arn. Arb. 22: 571, 1941.

Little, Am. Midl. Nat. 29: 493-508, 1943.

Fernald, Rhodora 49: 194-196, 1947.

Lawrence, Gent. Herb. 8(1): 25, 1949. 
303. Hernandria Plum. ex L., Gen. Pl., ed. 5, 421, 1754. Hernandia Plum. ex L., Sp. Pl. 981, index, 1753.

304. Acer rubrum L., Sp. Pl. 1,055, 1753. A. carolinianum Walt., Fl. Carol. 251, 1788.

Rufacer rubrum (L.) Small, Man. S. E. FI. 826, 1,505, 1933. R. carolinianum (Walt.) Small, Man. S. E. Fl. 826, 1,505, 1933.

A. stenocarpum Britt. in Britt. \& Shafer, N. Am. Trees 647, fig. 598, 1908.

A. stenocarpum Ettinghausen, K. Bayer. Akad. Wiss.

München, Denkschr. 50: 20, pl. 31, fig. 10-12, 1885.

305. Iresine celosia L. grex lutescens Suesseng., Bot.

Staatssammlung München, Mitt. 4: 106, 1952.

306. Terminalia mollis Teysmann \& Binnendijk, Cat. Bot. Gard.

Buitenzorg 252, 1855; 237, 1866.

T. mollis M. Laws. in Oliver, Fl. Trop. Afr. 2: 417, 1899.

T. mollis (Presl) Rolfe, Jour. Bot. Brit. For. 23: 212, 1885.

Pentaptera mollis Presl, Epim. Bot. 214, 1852; Kgl. Böhm.

Gesell. Wiss., Abh., V, 6: 574, 1851.

See van Steenis, Jard. Bot. Buitenzorg, Bul. III, 13: 117119, 1933.

307. Aconitum Napellus L. $\beta$ alpinum Regel, Lusus $\alpha$ ambiguum

(Turcz.) Regel, Ann. Sci. Nat. Bot. IV, 16: 150, 1862. A. ambiguum Turcz., Fl. Baic. Dah. 1: 81, 1842.

308. Rafinesquia Raf., Sylva Tellur. 79, 1838.

Rafine squia Raf., Fl. Tellur. 1: 17, (1836)=[1837]; 2(1):

96, $(1836)=$ [1837].

Rafine squia Raf., Fl. Tellur. 3: 82, (1836) =[1837].

Rafinesquia Nutt., Am. Phil. Soc., Trans. II, 7: 429, 1841.

309. Hydrocotyle cordata Walt., Fl. Carol. 113, 1788.

Anonymos aquatica Walt., Fl. Carol. 108-109, 1788.

Nymphodes aquaticum (Walt.) Ktze., Rev. Gen. Pl. 2: 429,

1891; 3: 202, 1893.

Nymphoides [Tourn.] Medic., Phil. Bot. 1: 35, 1789.

See Fernald, Rhodora 42: 297-298, 1940.

310. Pinus palustris Mill., Gard. Dict., ed. 8, Pinus No. 14, 1768. 
P. palustris Willd., Sp. Pl. of Linnaeus, ed. 4 by Willd., 4(1): 499, 1805.

$P$. australis Michx. f., Hist. Arbr. For. Amér. Sept. 1: 6485, pl. 6, 1810.

See Fernald, Rhodora 50: 181-186, 241-249, 1948.

Little, Phytologia 2: 457-458, 1948.

311. Atacciae cristatae (Jack) Haudricourt, Rev. Bot. Appliq. 22: 73, 1941.

A. schizocapsae Haudr., Rev. Bot. Appliq. 22: 74, 1941.

312. Gardenia Remyi Mann, Am. Acad. Arts Sci., Proc. 7: 171, 1867.

G. Remyi St. John, Brittonia 6: 438-444, fig. 4, a-f, 5, 7, 1949.

313. Microstylis (Nutt.) Lindl., Orchid. Scel. 17, 1826. Microstylis (Nutt.) Eaton, Man. Bot. N. Am., ed. 3, 353, 1822.

Malaxis Soland. ex Sw., Nov. Gen. et Sp. Pl. seu Prodr. 119, 1788.

See Taxon 3: 240, 1954.

314. Oenothera Hookeri T. \& G. ssp. eu-Hookeri Munz, El Aliso 2(1): $11,1949$.

O. Hookeri T. \& G. var. eu-Hookeri Munz, El Aliso 2(1): 11, adnot., 1949.

315. Broussaisia arguta Gaud. var. arguta Fosb. forma arguta

Fosb., Bishop Mus., Occas. Pap. 15: 56, 1939.

316. Claytonia depressa (Gray) Suksd. var. latifolia Suksd.,

Deutsch. Bot. Monatschr. 16: 222, 1898.

Montia latifolia Suksd., Deutsch. Bot. Monatschr. 16: 222,

1898.

317. Rudbeckia purpurea. *serotina I Nutt., Fraser's Cat. 1813; repr. in Pittonia 2: 118, 1890.

R. serotina Nutt., Acad. Nat. Sci. Phila., Jour. 7: 80-81, 1834.

See Shinners, Rhodora 57: 290-293, 1955;

Graustein, Rhodora 58: 20-22, 1956;

Cronquist et al., Rhodora 58: 23-24, 1956;

Shinners, Rhodora 58: 281-289, 1956;

Cronquist, Rhodora 59: 100, 1957. 
318. Thryocephalon nemorale J. R. \& G. Forst., Char. Gen. 129, pl. 65, 1776.

Kyllinga nemoralis (J. R. \&. G. Forst.) Dandy in Hutchinson

\& Dalziel, Fl. W. Trop. Afr. 2: 486-487, 1936.

K. monocephala Rottb., Descr. et Icon. Nov. Pl. 13, pl. 4,

fig. 4, 1773, partim.

Cyperus Kyllingia Endl., Cat. Hort. Acad. Vindob. 1: 94,

1842.

Scirpus Cephalotes L., Sp. Pl., ed. 2, 1: 76, 1762.

Schoenus Cephalotes (L.) Rottb., Descr. et Icon. Nov. PI. 61, pl. 20, 1773.

319. Victoria Regia Lindl., Victoria Regia 3, col. pl., 1837. Nymphaea Victoria Schomburgk ex Lindl., Monogr. 3, 1837. Euryale amazonica Poeppig, Notizen aus den Gebiete der

Natur- und Heilkunde, ed. Froriep 35: 131-132, 1832. Victoria regina Schomb. ex J. E. Gray, Mag. Zool. \& Bot.

2: 440, 1838.

V. regalis Schomb., Mag. Zool. \& Bot. 2: pl. 12, 1838.

Anneslea amazonica Presl, Bot. Bemerk. 103, 1844.

V. amazonum Klotzsch, Bot. Zeit. 5: 245, 1847.

$V$. reginae Hook., Kew Jour. 2: 314, 1850.

V. Cruziana D’Orbigny, Ann. Sci. Nat. Bot. II, 13: 57, 1840.

V. amazonica (Poepp.) Sowerby, Ann. \& Mag. Nat. Hist. II,

6: $310,1850$.

320. Charpentiera Gaud. in Freyc., Voy. Bot. Uranie 444, pl. 47, $48,(1826)=[1829]$.

Carpenteria Torr., Smithsonian Contr. 6: 12-13, pl. 7, 1854. Carpentaria Becc., Jard. Bot. Buitenz., Ann. 2: 128, 1885. Carpentiera Steud., Nomencl. Bot., ed. 2, 1: 344, 1840.

321. Abies Hill, Brit. Herb. 509-510, 1756. Abies Bertol., Amoen. Ital. 287, pl. 4, fig. 2, a,c, 1819.

322. Acoelorraphe Wendl., Bot. Zeit. 37: 148, 1879. Acoeloraphe Wendl. ex Becc., Webbia 2: 107, 1907. Acanthosabal Proschowsky, Gard. Chron. III, 77: 91-92, fig. 35-38, 1925.

Paurotis O. F. Cook, Torrey Bot. Club, Mem. 12: 21-23, 1902.

See Moore, Gent. Herb. 8: 209-215, 1951. 
323. Trematolobelia macrostachys (H. \& A.) Zahlbr. in Rock var. grandifolia Rock, Coll. Hawaii Publ., Bul. 2: 46, 1913. T. grandifolia Degener, Fl. Haw. fam. 339: 4/20/'34.

324. Macaranga Maudslayi Horne, A Year in Fiji 264, 1881; Baker in Linn. Soc. Lond., Bot., Jour. 20: 371, 1883; Pax \& Hoffm., Engler's Pflanzenreich IV, 147(7): 394, 1914. See A. C. Smith, Jour. Arn. Arb. 33: 390, 1952.

325. Albizzia calliandra Egler, Key to the Common Leguminosae of the Hawaiian Is., First Manuscript Ed. 34, 1940.

326. Torreya Arn., Ann. Nat. Hist. 1: 126-132, 1838.

T. californica Torr., New York Jour. Pharm. 3: 49-51, 1854. T. Myristica Hook., Curtis's Bot. Mag. 80: pl. 4,780, 1854. Tumion Raf., Good Book and Amenities of Nature 62-63, 1840.

Tumion californicum (Torr.) Greene, Pittonia 2: 195, 1891. Caryotaxus Zucc. ex Henkel \& Hochst., Synops. Nadelh. $365,1865$.

Foetaxus J. Nelson (as Senilis), Pinaceae 167, 1866. Torreya Spreng., Neue Entdeck. 2: 121-122, 1821.

Torreya Eaton, Man. Bot. N. Am., ed. 5, 420, 1829.

327. Brachycome Superspecies tenuiscapa G. L. Davis, Linn. Soc. N. S. W., Proc. 73(3): 152, 1948.

328. Andruris palawensis Tuyama, Bot. Mag. Tokyo 52: 63, 1938. A. elegans Giesen, in Engler's Pflanzenreich 18: 25, fig. 5, 1-4, 1938.

See Hosokawa, Jour. Jap. Bot. 16: 540, 1940.

329. Hosta japonica (Thunb.) Voss, Vilmorin's Blumengaertn. 1: 1,070, 1896.

Aletris japonica Thunb., Reg. Soc. Sci. Upsal., Nov. Acta

3: 208, 1780.

Hemerocallis Japonica Thunb., Fl. Japon. 142-143, 1784. Hemerocallis lancifolia Thunb., Linn. Soc. Lond., Trans. 2: 335, 1794.

Hosta lancifolia (Thunb.) Tratt., Archiv Gewächsk. 1: 55, 1812.

See Stearn, Gard. Chron. III, 89: July 11, 27; July 18, 48-49; Aug. 1, 88-89; Aug. 8, 110, 1931.

Bailey, Gent. Herb. 2: 129-131, 1930; 2: 435, 1932. 
330. Rhynchospora lavamum Gaud., Voy. Freyc. Coquille 415, 1826.

R. glauca Vahl var. chinensis (Boeck.) C. B. Clarke forma

lavarum (Gaud.) Kük. ex Skottsb., Göteborg Trädg.,

Meddel. 2: 213, 1925.

R. glauca Vahl ssp. lavarum (Gaud.) Kük., Engler's Bot.

Jahrb. 75: 150, 1950.

Schoenus rugosus Vahl, Eclog. Am. 2: 5, 1798.

R. rugosa (Vahl) Gale, Rhodora 46: 275-278, pl. 835, fig.

$1 \mathrm{~A}, 1944$.

331. Biotia D. Don in Lamb., Pinet., ed. 2, 2: 129, 1828.

Biotia DC., Prodr. 5: 264, 1836.

Biotia Cass., Dict. Sci. Nat. 34: 308, 1825.

Biotia Lamb., Descr. Gen. Pinus 2: 129, 1832.

332. Frullania helleri Stephani, Herb. Boissier, Bul. 5: 845-846, 1897.

F. helleri Steph. in Rechinger, Bot.-Zool. Ergebn. Samoa,

Neuguinea, Salomoninseln 3: 19, 1910.

333. Saurauia roseotincta R. E. Schultes, Harvard Univ., Bot.

Mus. Leafl. 16(4): 83-86, 1953.

See Rickett, Taxon 4: 185-188, 1955.

334. Selaginumus Herter, and Selagina Herter, Die Bärlappgewächse, 21, 22, 27, 1939.

335. Picea Sitchensis (Bong.) Carr., Traité Gén. Conif. 260-261, 1855.

Pinus sitchensis Bong., Acad. Imp. Sci. St. Petersbourg,

sér. 6, Sci. Math. Phys. Nat., Mem. 2: 164, 1832

(August).

Pinus Menziesii Dougl. ex D. Don in Lamb., Descr. Genus

Pinus, ed. 3, (octavo), 2: unnumbered page between Pp. 144 and 145, 1832.

336. Gifola Cass., Soc. Philom., Paris, Bul. Sci. 142, 1819. Filago P. Loefling in L., Gen. Pl., ed. 5, 397, 1754; Sp. Pl. 927, 1753.

Ifloga Cass., Soc. Philom., Paris, Bul. Sci. 142, 1819. Oglifa Cass., Soc. Philom., Paris, Bul. Sci. 143, 1819. Lofgia de Toni, Archivio Botanico 14(3-4): 19, 1938. Logfia Cass., Soc. Philom., Paris, Bul. Sci. 143, 1819. 
337. Rivinia humilis L., Sp. Pl. 1: 121-122, 1753.

R. humilis L. $\alpha$ canescens L., Sp. Pl. 122, 1753.

338. Putranjiva formosana Kanehira \& Sasaki, in Shimada, Nat.

Hist. Soc. Formosa, Trans. 24: 83, 1934.

Drypetes formosana (Kanehira \& Sasaki) Kanehira, Formosan Trees, rev. ed., 336, fig. 292, 1936.

339. Amorpha nana Nutt., Fraser's Cat. 1813; repr. in Pittonia 2:

116, 1890.

A. microphylla Pursh, Fl. Am. Sept. 466-467, 1814.

See Shinners, Rhodora 57: 290-293, 1955;

Cronquist et al., Rhodora 58: 23-24, 1956;

Shinners, Rhodora 58: 281-289, 1956;

Graustein, Rhodora 58: 20-22, 1956.

Cronquist, Rhodora 59: 100, 1957.

340. Panicum L., Gen. Pl., ed. 5, 29, 1754; Sp. Pl. 55, 1753.

See Hitchcock \& Chase, U. S. Natl. Herb., Contr. 15: 11$16,1910$.

341. Klibea Sherff, Field Mus. Nat. Hist., Bot Ser. 8(6): 423, 1932.

342. Callicarpa resinosa Wright \& Moldenke, Fedde Repert. Sp.

Nov. 33: 142-143, 1933.

C. resinosa Wright \& Moldenke ex Moldenke, Fedde Repert.

Sp. Nov. 40: 77-78, 1936.

343. Nani(a) lutea (Gray) Heller, Minn. Bot. Stud. 1: 867-868, 1897.

Metrosideros lutea Gray, Bot. U. S. Expl. Exped. 15: 560, 1854.

Nani Adans., Fam. Pl. 2: 88, 1763.

Nania Miq., F1. Ind. Bat. 1(1): 399, 1855.

Metrosideros Banks ex Gaertn., Fruct. 1: 170, pl. 34, fig. 1, 1788.

344. Phyllites Brongn., Mus. Hist. Nat. Paris, Mém. 8: 237-238, 1822.

Quercus mohavensis Axelrod, Carnegie Inst. Wash., Publ.

516: 99, pl. 8, fig. 1, 1939.

Phyllitis dentatus Axelrod, Carnegie Inst. Wash., Publ.

516: 128, pl. 12, fig. 9, 1939.

See Lamotte, Geol. Soc. Am., Mem. 51: 294, 1952. 
345. Oxalis Wrightii Gray, Pl. Wright., in Smithsonian Inst., Contr. Know. 3(5): 27, 1850.

346. Alisma Damasonium L., Sp. Pl. 343, 1753.

Damasonium Alisma Mill., Gard. Dict. ed. 8, Alisma No. 1, 1768.

Damasonium damasonium (L.) Aschers. \& Graebn., Fl. Mitteleurop. 1: 389, 1897.

D. Stellatum Thuill., Fl. Paris, ed. 2, 186, 1799.

347. Bicornes L., Gen. Pl., ed. 6, sixth page after the index, 1764.

See H. F. Copeland, Madroño 9: 65, 96, 1947.

348. Aciphylla J. R. \& G. Forst., Char. Gen. 135-136, pl. 68, 1776.

Laserpitium Aciphylla (J. R. \& G. Forst.) Forst. f., Fl. Ins. Aust. Prodr. 22, 1786.

349. Holcus L., Gen. Pl. 469, 1754; Sp. Pl. 1,047-1,049, 1753. See Hitchcock, Gen. Grasses U. S., U. S. Dept. Agric., Bul. 772: 266, 1920; Man. Grasses U. S., U. S. Dept. Agric., Misc. Publ. 200: ed. 2, 957, 1950; Shinners, Baileya 4: 141-142, 1956.

350. Urophyllum Johannis Winkleri Merr., Inst. Allgem. Bot. Hamburg, Mitt. 7(4): 289-290, 1937.

351. Potamogeton Purshii Tuckerm., Am. Jour. Sci. II, 6: 228, 1848.

352. Hedysamum Macquenzii Richards. forma canescens

Fedtschenko, Hort. Petrop., Acta 19: 274, 1901.

H. Mackenzii Richards. var. canescens Fedtschenko, Hort.

Petrop., Acta 19: 362, 1901.

H. Mackenzii Hook., Fl. Bor.-Am. 1: 155-156, 1831.

H. boreale Nutt. var. cinerascens (Rydb.) Rollins, Rhodora 42: 234, 1940.

H. cinerascens Rydb., New York Bot. Gard., Mem. 1: 257, 1900.

353. Pinus baumani Read, Carnegie Inst. Wash., Publ. 416: 1112, 1933.

P. bowmanii Read, Carnegie Inst. Wash., Publ. 416: pl. 3, fig. 1-6, 1933.

See Lamotte, Geol. Soc. Am., Mem. 51: 253, 1952. 
354. Pandanus Hermsianus Martelli, Univ. Cal if. Publ. Bot. 13(7): 145-146, pl. 12, 1926.

$P$. fanningensis Martelli, Roy. Soc. Queensland, Proc.

45(4): 23, 1933.

P. spiralis R. Br., Prodr. 341, 1810.

355. Fouilloya Gaud., Bot. Voy. La Bonite, pl. 26, fig. 1-7, 2124, $(1844-1866)=[1841]$.

356. Viorna (Spach) Gray, Syn. Fl. N. Am. 1(1): 5, 1895.

Viorna Pers. in Reichb., Handb. Natürlich. Pflanzensyst.

277, 1837.

Viorna Pers., Syn. Pl. 2: 98, 1807.

Urnigerae Lavalée, Clematites Gr. Fl. 47, 1884.

See Erickson, Mo. Bot. Gard., Ann. 30: 12, 1943.

357. Amum Colocasia L., Sp. Pl. 965, 1753.

Colocasia Antiquorum Schott in Schott \& Endl., Meletem.

Bot. 1: 18, 1832.

Caladium Colocasia (L.) W. F. Wight, U. S. Natl. Herb.,

Contr. 9: 208, 1905.

Arum esculentum L., Sp. Pl. 965, 1753.

Caladium esculentum (L.) Vent., Descr. Pl. Nouv. Jard.

Cels. pl. 30, 1800; Willd. in Linnaeus, Sp. Pl., ed. 4 by

Willd. 4: 489, 1805.

Colocasia antiquorum Schott var. $\epsilon$ esculenta (L.) Schott,

Syn. Aroid. 42, 1856.

Colocasia esculenta (L.) Schott in Schott \& Endl., Meletem.

Bot. 1: 18, 1832; Engler in DC., Monogr. Phan. 2: 491, 1879.

Colocasia esculenta (L.) Schott var. typica A. F. Hill, Harvard Univ., Bot. Mus., Leafl. 7(7): 116, 1939.

358. Eggelingia Summerh., Harvard Univ. Bot. Mus., Leafl. 14(9): 235-236, 1951.

359. Puccinia Polygoni amphibii Pers., Syn. Meth. Fung. 227228, 1801.

Uredo Polygoni Schum., Pl. Saell. 2: 233, 1803.

Puccinia Polygoni Alb. \& Schw., Consp. Fung. 127, 1805. Dicaeoma Polygoni-amphibii (Pers.) Arth., Ind. Acad. Sci.,

Proc. 1898: 184́, 1899.

See Jackson, Mycologia 19: 55, 1927. 
360. Dalechampia [Plum.] L., Gen. P1., ed. 5, 473, 1754.

361. Hysterium smilacis Schw., Naturf. Ges. Leipzig, Schr. 1:

49, 1822; Schw. in Fries, Syst. Mycol. 2(2): 586, 1823. Hysterographium smilacis (Schw.) Ellis \& Everh., N. Am.

Pyren. 709, 1892.

Gloniopsis smilacis (Schw.) Underw. \& Earle, Ala. Agric.

Exp. Sta., Bul. 80: 196, 1897.

G. Ellisii Cash, Mycologia 31: 294, 1939.

Hypoderma smilacis (Schw.) Sacc., Syll. Fung. 10: 789, 1883.

Hypodermopsis smilacis (Schw.) Cash, Mycologia 31: 293, 1939.

See Petrak, Sydowia 6: 290-292, 1952.

362. Lupinus Hellerae Heller, Torrey Bot. Club, Bul. 25: 265, 1898.

L. minimus Dougl. var. Hellerae (Heller) C. P. Sm. \& St.

John in St. John, Fl. S. E. Wash. 228, 1937.

L. Helleri Greene, Pittonia 4: 134, 1900.

363. Platyzomataceae Nakai, Natl. Sci. Mus. Tokyo, Bul. 29: 4, 1950.

364. Sagittaria calycina Engelm. in Torr., Mex. Bound. Surv. Bot.

2: $212,(1858)=[1859]$.

Lophiocarpus calycinus (Englem.) Micheli in DC., Monogr.

Phan. 3: 61-62, 1881.

Lophiocarpus Turcz., Soc. Imp. Nat. Moscou, Bul. 16: 55,

1843.

Lophotocarpus calycinus (Engelm.) J. G. Sm., Mo. Bot.

Gard., Rept. 6: 60, 1895.

Lophianthus calycinus (Engelm.) Micheli ex J. G. Sm.,

Torrey Bot. Club, Mem. 5: 25, 1894.

365. Agavoideae Pax in Engler \& Prantl, Nat. Pflanzenfam. IV, 2(5): 115, 1887.

Agaveae Endl., Gen. Pl. 181, 1836-1840.

Agavaceae Lotsy, Vorträge Bot. Stammesgesch. 3: 806,

1911.

366. Corydalis aurea Willd., Enum. Hort. Reg. Bot. Bero 1. 2:

$740,1809$. 
Fumaria aurea Mühl. in Willd., Enum. Hort. Reg. Bot. Berol. 2: $740,1809$.

C. aurea Willd. var. $\alpha$ typica Regel, Acad. Imp. Sci. St.

Petersb., Mém. VII, 4: 19, 1861.

C. aurea Willd. ssp. aurea Ownbey, Mo. Bot. Gard., Ann.

34: 229, 1947.

367. Succuta (Des Moulins) Yuncker, Illinois Biol. Monogr.

6(2-3): 21, 1921.

Eucuscuta Engelm., Acad. Sci. St. Louis, Trans. 1: 460, 1859.

Succuta Des Moulins, Études Org. Cusc. 74, pl. 17, 1853.

Schrebera L., Sp. Pl., ed. 2, 1,662, 1763.

Lepimenes Raf., F1. Tellur. 4: 91, 1836.

368. Cyrtandra Wilderi St. John \& Storey, Bishop Mus., Occ. Pap. 20(6): 88, 1950.

C. Lessoniana Gaud. var. $\beta$ Hbd., Fl. Haw. Is. 331, 1888.

C. Lessoniana Gaud. var. stenoloba Skottsb., Göteborg Bot. Trädgård, Med. 10: 173, 1936.

369. Conyza Japonica (Thunb.) Less., Syn. Gen. Composit. 204, 1832; DC., Prodr. 5: 382, 1836.

Erigeron Japonicum Thunb., Fl. Jap. 312-313, 1784.

370. Elymus mollis Trin. in Spreng., Neu. Entd. 2: 72, 1821.

$E$. arenarius L. $\beta$ villosus E. Mey., Pl. Labrad. 20, 1830.

E. ampliculmis Provanch., F1. Canad. 2: 706, 1862.

E. capitatus Scribn., J. S. Dept. Agric., Div. Agrost., Bul.

11: 55, pl. 14, 1898.

E. arenarius L., Sp. Pl. 83, 1753.

$E$. dives Presl, Rel. Haenk. 1: 265, 1830.

E. arenarius L. ssp. mollis (Trin.) Hultén, Kungl. Svenska

Vetenskapsakad., Handl. III, 5(1): 153-155, 1927.

See St. John, Rhodora 17: 98-103, 1915.

Hitchcock, U. S. Dept. Agric., Misc. Publ. 200: 249, $845,1935$.

Löve, Soc. Fauna \& Fl. Fennica, Acta 72(15): 6, 1955.

371. Plectocomiopsis dubius Becc., Roy, Bot. Gard. Calcutta, Ann. 12(2): 56, 58, pl. 37, 1918. 
372. Melicocca L., Sp. Pl., ed. 2, 495, 1763.

Melicoccus P. Br., Civ. Nat. Hist. Jam. 210-211, 1756.

See Taxon 3: 241, 1954.

373. Ficus velascoi (Merr.) Sata, Monogr. Stud. Ficus, Taihoku

Imp. Univ., Fac. Agric., Inst. Hort. \& Econ. Bot.,

Contr. 32(1): 242, 275, 1944.

374. Panax Schin-seng Nees, Icon. Pl. Med. Suppl. No. 70, fasc.

5, pl. 16, unnumbered, 1833.

P. Ginseng C. A. Meyer, Repert. Pharm. Chem., Jahrg.

1842(8): 524, 1842; Phys. Math. Acad. St. Petersburg,

Bul. 1: 340, 1843.

Aralia Ginseng (C. A. Meyer) Baill., Hist. Pl. 7: 197, 1880.

See Hill, Harvard Univ. Bot. Mus., Leafl. 10: 163, 1942.

375. Betula Andrewsii A. Nels., Bot. Gaz. 43: 279-281, 1907. B. papyrifera Andrewsii (A. Nels.) F. P. Daniels, Univ.

Mo. Stud., Sci. Ser. 2: 101, 1911.

B. occidentalis Hook. $\times$ papyrifera Marsh., Froiland, Evolution 6(3): 281, 1952.

See Butler, Torrey Bot. Club, Bul. 36: 435, 1909.

376. $\times$ Veronicena Moldenke, Phytologia 5: 133, 1955.

$\times V . \times$ Haartmani Moldenke, Phytologia 5: 134, 1955.

Veronica $\times$ hybrida Haartm., Pl. Hybrid. 7-8, 1751.

Veronica hybrida L., Sp. Pl. 11, 1753.

Veronica $\times$ spuria Haartm., Amoen. Acad. 3: 35, pl. 2, 1756. Veronica spuria L., Sp. Pl. 10, 1753.

377. Larix laricina (Du Roi) K. Koch, Dendrol. 2(2): 263, 1873. Pinus Laricina Du Roi, Dissert. Inaug. Observ. Bot. 49-50, [1771].

378. Platycarya Sieb. \& Zucc., Akad. Muenchen, Abh. 3(3): 741743, pl. 5, fig. 1, 1843.

Petrophiloides Bowerbank, Foss. Fr. London Clay 43, 1840.

379. Syzygium Gaertn., Fruct. 1: 166, 1788.

Suzygium Gaertn., Fruct. 3: pl. 33, fig. 1, 1788.

380. Corynocarpus J. R. \& G. Forst., Char. Gen. 31-32, pl. 16, 1776.

381. Barleria [Plum.] ex L., Gen. Pl., ed. 5, 283, 1754. 
382. Panicum Mattamusketense Ashe, Elisha Mitchell Sci. Soc., Jour. 15: 45, 1898.

383. Horsfieldia Willd., Sp. Pl. of Linnaeus, ed. 4 by Willd., 4:

$872,1805$.

Horkelia C. \& S., Linnaea 2: 26, 1827.

384. Rooseveltia O. F. Cook, Smithsonian Misc. Coll. 98(7): 21-22, pl. 1-19, 1939.

385. Aesculus L., Gen. Pl., ed. 5, 500, 1754; Sp. Pl. 344, 1753. Esculus L., Gen. Pl., ed. 5, 161, 1754. Oesculus Neck., Elem. Bot. 2: 232, 1790.

386. Cyrtandra lillianae Setchell in Wilder, Bishop Mus., Bul. 86: 98, fig. 3, 1931.

387. Euodia Bailey ex Pritch., Hist. Infusoria 852, 1861. Euodia Gaertn., Fruct. 2: pl. 103, fig. 2, 1791. Euodia J. R. \& G. Forst., Char. Gen. 13, pl. 72, 1776. Evodea Kunth, Syn. Pl. 3: 327, 1824. Evodia Gaertn., Fruct. 2: 100, pl. 103, 1791.

388. Scolecotrichum Asclepiadis Ellis \& Everh., Erythea 1: 203, 1893.

389. Thermopsis pauciflora Thornber ex Larisey, Mo. Bot. Gard., Ann. 27: 252, 1940.

390. Melochia concatenata L., Sp. Pl. 675, 1753. M. corchorifolia L., Sp. Pl. 675, 1753.

See Mast. in Hook. f., Fl. Brit. India 1: 374, 1874. Merrill, Am. Phil. Soc., Trans. 24(2): 264, 1935.

391. Sassafras variifolium (Salisb.) Ktze., Rev. Gen. PI. 2: 574 , 1891.

Laume Variifolia Salisb., Prodr. Hort. Chap. Allerton 344, 1796.

Laumes Sassafras L., Sp. Pl. 371, 1753.

Laumes albida Nutt., Gen. N. Am. Pl. 1: 259-260, 1818.

Tetranthera albida (Nutt.) Spreng., Syst. Veg. of Linnaeus, ed. 16 by Spreng., 2: 267, 1825 .

Sassafras officinalis Nees \& Eberm., Handb. Med.-Pharm.

Bot. 2: 418, 1831.

S. albidum (Nutt.) Nees, Syst. Laurin. 490, 1836.

S. triloba Raf., Autikon Bot. 85, 1840. 
S. Sassafras (L.) Karst., Deut. Fl. Pharm.-Med. Bot. 505, $(1882)=[1881]$.

S. albidum var. molle (Raf.) Fern., Rhodora 38: 179, 1936. S. variifolium (Salisb.) Ktze. var. albidum (Nutt.) Fern.,

Rhodora 15: 16, 1913.

S. albida (Nutt.) Nees var. glauca Nieuwl., Am. Midl. Nat. 3: $347,1914$.

S. officinale Nees \& Eberm. var. albidum (Nutt.) Blake, Rhodora 20: 99, 1918.

392. Verrucaria Webber ex Wiggers, Prim. Fl. Holsat. 85, 1780. Verrucariomyces Thomas ex Cif. \& Tom., Inst. Bot. Lab.

Crittog. Pavia, Atti V, 10: 31, 1952; Thomas, Beitr.

Kryptogamenfl. Schweiz 9(1): 171, 1939.

393. Swertia L., Gen. Pl., ed. 5, 107, 1754; Sp. Pl. 226-227, 1753.

Svertia L., Sp. Pl. index, 1753.

394. Poa amboinica L., Mant. Alt. 557, 1771.

Phoenix amboinica montana Rumph., Herb. Amboin. 6: 19, pl. 7, fig. 3, 1750.

Andropogon amboinicus (L.) Merr., Interpret. Rumph. Herb. Amboin. 88, 1917.

See Fischer, Kew. Bul. 398-400, 1934.

Airy Shaw, H. K., Kew Bul. 36, 1947.

Furtado, Gardens' Bul. 9: 246, 1937.

395. Pisum maritimum L. $\beta$ glabmum Ser. in DC., Prodr. 2: 368, 1825.

Lathyrus japonicus Willd. var. glaber (Ser.) Fern., Rhodora 34: 181, 1932.

396. Quercus Margaretta Ashe in Small, F1. S. E. U. S. 355, 1903; ed. 2, 355, 1913.

Q. minor (Marsh.) Sarg. var. Margaretta Ashe, Elisha

Mitchell Sci. Soc., Jour. 11: 94, 1894.

Q. stellata Wang. var. Margaretta (Ashe) Sarg., Trees \&

Shrubs 2: 219, pl. 185, 1913.

397. Eugenia waianensis Degener, Fl. Haw. 273: with figure, $7 / 15 / ' 32$.

E. rariflora Benth. var. parvifolia Hbd., Fl. Haw. Is. 129,

1888.

E. parvifolia DC., Prodr. 3: 266, 1828. 
398. Dentaria digitata Lam., Encyc. Méth. Bot. 2: 268, 1786. Cardamine hyperborea O. E. Schulz, Engl. Bot. Jahrb. 32: 550, 1903. C. Richardsonii Hultén, Lunds Univ., Årssk. 41: 838, 1945. C. digitata Richards. in Franklin, Journey Shores Polar Sea, Bot. Append. 743, 1823.

See Rollins, Rhodora 54: 260, 1952.

399. Quercus Lowilliamsi Muller, Madroño 10: 138-139, pl. 11, 1950.

400. Pithecellobium Mart., in v. Schrank \& Mart., Hort. Reg.

Monac. 188, 1829.

Pithecollobium Mart., Flora 20(2), Beibl: 114, 1837.

Zygia P. Browne, Civ. Nat. Hist. Jamaica 279, pl. 22, fig.

3, 1789.

Zygia Boehm. ed. I.udw., Def. Gen. Pl. 72, 1760.

Siderocarpus Small, New York Bot. Gard., Bul. 2: 91, 1901. Havardia Small, New York Bot. Gard., Bul. 2: 91-92, 1901. Ebenopsis Britton \& Rose, N. Am. Fl. 23(1): 33, 1928.

401. Epidendrum Flos aëris L., Sp. Pl. 952, 1753.

402. Cyrtandra Hosakae St. John, Bishop Mus., Occas. Pap. 20(6): 82, 1950.

403. Cyrtandra Lessoniana Gaud., Bot. Voy. Freyc. Uranie 447, $(1826)=[1829] ;$ Atlas, pl. 54, 1826.

C. Lessoriana Gaud. sensu Wawra, Flora 30: 561 (reprint, p. 17), 1872.

404. Coprosma ochracea W. R. B. Oliver, var. kaalae St. John in W. R. B. Oliver, Bishop Mus., Bul. 132: 157-158, fig. 53, 1935.

405. Leucanthemum [Tourn.] Adans., Fam. Pl. 2: 127, 1763. 406. Rosineae Engler, Nat. Pflanzenfam., Nachtr. 349, 1897. 407. Cyclospermum leptophyllum (Pers.) Sprague, Jour. Bot. Brit. For. 61: 131, 1923.

Pimpinella leptophylla Pers., Syn. Pl. 1: 324, 1805. Apium leptophyllum (Pers.) F. Muell. ex Benth., Fl.

Austral. 3: 372, 1866.

Sison Ammi Jacq., Hort. Vindob. 2: 95, pl. 200, 1773. Apium Ammi (L.) Urban, Fl. Bras. 11(1): 341, 1879. 
See Constance in Kearney \& Peebles, U. S. Dept. Agric., Misc. Publ. 423: 645, 1942.

408. Eriosorus Fée, Gen. Fil. 152, $(1850-1852)=[1850]$.

See Pichi-Sermolli, Webbia 9: 361, 362, 1953.

409. Oedogonium Kurzii Zeller var. ovatum H. Silva, Torrey Bot. Club. Bul. 80: 342-343, 1953.

410. Conioselinum chinense (L.) BSP., Prel. Cat. New York 22, 1888.

Athamanta chinensis L., Sp. Pl. 245, 1753.

Selinum canadense Michx., Fl. Bor.-Am. 1: 165, 1803.

Conioselinum ? canadense (Michx.) T. \& G., Fl. N. Am. 1:

$619,1840$.

See Hultén, Lunds Univ. Årsskr. n. f. II, 43(1): 1,175, 1946.

411. Cupressus M'Nabiana A. Murr., Edinb. New Phil. Jour. n.s.

1: 293, pl. 11, 1855; repr. 12-13, pl. 11, 1855.

412. Sida pseudo-potentilloides H. Monteiro f., Lilloa 17: 516, 1949.

S. potentilloides K. Schum. in Mart., Fl. Bras. 12(3): 334, 1891.

S. potentilloides St. Hil., F1. Bras. Merid. 1: 110, 1825.

413. Dioscorea sativa L., Sp. Pl. 1,033, 1753.

D. sativa Thunb., Fl. Jap. 151, 1784.

D. bulbifera L. var. sativa Prain \& Burkill, Calcutta Roy.

Bot. Gard., Ann. 14(1): 113, 117, 122-123, 132, 1936;

14(2): pl. 49-50, 1936.

414. Urera sandwicensis Wedd. $y$ mollis Wedd. in DC., Prodr.

16(1): 93, 1869.

U. glabra (H. \& A.) Wedd. $\beta$ mollis Wedd., Mus. Hist. Nat.

Paris, Arch. 9: 149, 1856.

415. $\times$ Tritordeum Aschers. \& Graebn., Syn. Mitteleur. Fl. 2(1):

748, 1902.

$\times$ Agroelymus G. Camus in A. Camus, Mus. Natl. Hist. Nat.

Paris, Bul. 6: 538, 1927.

$\times$ Elymopyrum de Cugnac, Soc. Hist. Nat. Ardennes, Bul. 33:

1938.

Agropyron repens $\times$ Elymus mollis J. Adams, Can. Field

Nat. 50: 117, 1936. 
$\times$ Agroelymus Adamsii Rousseau, Nat. Can. 69: 99, 1942. $\times$ Agroelymus jamesensis Lepage var. anticostensis Lepage,

Nat. Can. 79: 247, 1952.

$\times$ Agroelymus Adamsii Rousseau, Jard. Bot. Montreal, Mem.

29: 16-20, 1952.

416. Zygnema novae-caesareae Habeeb, Rhodora 56: 41-42,

1954.

See Silva, Univ. Calif., Publ. Bot. 25: 253, 1952.

417. Trifolium procumbens L., Sp. Pl. 772-773, 1753.

T. filiforme L., Fl. Suec., ed. 2, 261-262, 1755.

T. dubium Sibth., Fl. Oxon. 231, 1794.

T. minus Sm. in Relhan, Fl. Cantabr., ed. 2, 290, 1802.

T. procumbens L., Fl. Suec., ed. 2, 261, 1755.

T. campestre Schreb. in Sturm, Deutschl. Fl. (1) heft. 16:

pl. 13 unpaged, 1804.

T. agrarium L., Fl. Suec., ed. 2, 261, 1755.

See Hermann, U. S. Dept. Agric., Agric. Monogr. 22: 11,

1954.

418. Yucca glauca Nutt., Fraser's Cat. 1813; repr. Pittonia 2:

119, 1890.

Y. angustifolia Pursh, Fl. Am. Sept. 1: 227, 1814.

See Shinners, Rhodora 57: 290-293, 1955;

Graustein, Rhodora 58: 20-22, 1956;

Cronquist et al., Rhodora 58: 23-24, 1956;

Shinners, Rhodora 58: 281-289, 1956;

Cronquist, Rhodora 59: 100, 1957.

419. Nummularia Tul., Select. Fung. Carpolog. 2: 42-43, 1863. Nummularia S. F. Gray, Nat. Arr. Brit. Pl. 2: 300, 1821. Kommamyce Nieuwl., Am. Midl. Nat. 4: 375, 1916.

420. Aralia Necker, Elem. Bot. 1: 159, 1790. Aralia [Tourn.] L., Gen. Pl., ed. 5, 134, 1754.

421. Anthoceros megalospermus Gottsche, Bot. Zeit. 16, Beilage: 19, 1858.

A. megalosporus Gottsche, Bot. Zeit. 16, Beilage: 48, 1858.

422. Hypnomonas pleiopyrenigemum Moewus, Bot. Notis. 1953: 406-409, fig. 3, 1953.

423. Anthoceros Havaiensis Reichardt, K. K. Wien Akad., Sitzungsber, Abt. 1, 75: 562, 1877. 
A. hawaiensis Reich. ex Stephani, Herb. Boiss., Bul. 5: $841,1897$.

Aspiromitus hawaicus (Reich.) Steph., Sp. Hepat. 5: 967, 1916.

424. Liabum (Sinclairia) columbianum Klatt, Engl. Bot. Jahrb.

8: $47,1887$.

Gynoxys columbiana (Klatt) Hieron., Engl. Bot. Jahrb. 28:

631, 1901.

G. columbian (Klatt) Hieron. ex Cuatr., Fieldiana, Bot. 27(2): 17, 1951.

425. A ster arcticus Rupp., Fl. Ienensis 144, 1726.

426. Galapagoa Hook f., Linn. Soc. Lond., Proc. 1: 277, (1849)= [1845].

427. Spirotecoma Baill., Hist. Pl. 10: 49, 1891.

428. Migandra O. F. Cook, Natl. Hort. Mag. (Am.) 22: 142 in obs., 152, 1943.

429. Melaleuca Leucadendra (L.) L., Mant. Pl. 1: 105, 1767. Myrtus Leucadendra Stickm., Herb. Amb. 9, 1754; Amoen.

Acad. 4: 120, 1759.

Myrtus Leucadendra L., Syst. Nat., ed. 10, 2: 1,056, 1759. Melalevca Leucadendra (L.) L., Syst. Nat., ed. 13, 2: 509,

1770.

Melaleuca Leucadendron (L.) L. ex L. f., Suppl. Pl. 342, 1781.

See Little, Check List Nat. Nat. Trees U. S., U. S. Dept., Agric., For. Serv. 160, 1944; U. S. Dept. Agric., Agric. Harıdbook 41: 239, 1953.

430. Aspergillus mangini (Mangin) Thom \& Raper, Manual Aspergilli 127, 1945.

Eurotium herbariorum Link ser. minor Mangin, Ann. Sci.

Nat. Bot. IX, 10: 366-370, 1909.

A. minor (Mangin) Thom \& Raper, U. S. Dept. Agric., Misc.

Publ. 426: 27-29, 1941.

Sterigmatocystis minor Bainier, Soc. Bot. France, Bul. 27:

30-31, 1880.

431. Amphoradenium haalilioanum (Brack.) Copel., Gen. Fil. 220, 1947. 
Polypodium Haalilioanum Brack., U. S. Expl. Exped. Bot. 16: 5-6, 1854; Atlas 16: pl. 1, fig. 4, 1854. P. subpinnatifidum (Bl.) Hook., Sp. Fil. 4: 177, 1862. Grammitis subpinnatifidum (Bl.) Bl., Fil. Jav. 118, pl. 49, fig. 2, 1830.

P. subpinnatifidum Bl., Enum. Pl. Jav. 129, 1827.

432. Cuphea P. Browne, Civ. Nat. Hist. Jamaica 216, 1756. Balsamona Vand., Fasc. Pl. 15, 1771.

Dipetalon Raf., Sylva Tellur. 103, 1838.

Duvernaya Desp. in DC., Prodr. 3: 86, 1828.

433. Xylaria Cornu Dorcas Lloyd, Myc. Writ. 7: 1,206, pl. 249, fig. 2,498, 1924.

$X$. cornu dorcas Lloyd emend. Stevenson \& Cash, Lloyd

Libr. \& Mus., Bul. 35 (Mycol. Ser. 8): 13, 1936.

434. Schadjaret elharneb Forsk., Fl. Aegypt. Arab. 195, 1775.

435. Kosaria Forsk., Fl. Aegypt. Arab. 164-165, 1775.

436. Stephanospermum stewarti J. W. Hall, Bot. Gaz. 115: 354356, fig. $21-28,1954$.

437. Volvox capensis Pocock, S. Afr. Mus., Ann. 16(3): 441-447, repr., n. d.

438. Verbena litoralis HBK., Nov. Gen. et Sp. Pl. 2: 276, pl. $137,1817$.

Senecio littoralis Gaud., Ann. Sci. Nat. I, 5: 104, 1825. Ranunculus litoralis Phil., Linnaea 28: 663, 1856.

Carex littoralis Schwein., Lyc. New York, Ann. 1: 70, 1824.

439. Budleia Houston ex Adanson, Fam. Pl. 2: 224, 1763.

Budleja L., Gen. Pl., ed. 1, 1: index, 1737; 2: 95, and index, 1737.

Buddleja Houston ex L., Hort. Cliffort. 35, 1737; Gen. Pl., ed. 1, 1: 26, 1737; ed. 5, 51, 1754; ed. 6, 57, 1764; Sp. Pl. ed. 1, 112, 1753; Syst. Nat. of Linnaeus, ed. 12 by Beckmann, 2: 56, index, 1772.

Buddleia L., Syst. Nat., ed. 13, 2: 121, index, 1770; Benth. in DC., Prodr. 10: 433, 436, 1846; B. \& H., Gen. Pl. 2(2); 793, 1876; Solereder in Engler \& Prantl, Nat. Pflanzenfam. 4(2): 47, 1897.

Buddlea L., ex Spreng. in Syst. Veg. of Linnaeus, ed. 16 by Spreng. 1: 428, 975, 1825. 
Buddleya L. ex Franch. \& Savat., Enum. Pl. Jap. 1: 322, 1874.

Budlaea Sw., Observ. Bot. 47, 1791.

Budlea L. ex St. Hil., Expos. Fam. Nat. Pl. 1: 272, 1805.

See Nakai, Natl. Sci. Mus. (Tokyo), Bul. 29: 73, 1950.

Rickett, Taxon 4: 185-188, 1955.

440. Peckifungus Ktze., Rev. Gen. Pl. 2: 864, 1891.

441. Candida Berkh., De Schlimmelg. Monilia 41, 1923.

442. Dendrophthora Willtreleasii Stehlé, Soc. Bot. France, Bul. (Mem. 1953-1954): 32, 1954.

443. Jungermannia trichophylla L., Sp. Pl. 1,135, 1753.

Blepharostoma trichophyllum (L.) Dumort., Rec. d'Obs. 18, 1835.

444. Hypnum Stereodon virginianus Brid., Bryol. Univ. 2: 576, 1827.

445. Isotoma muncinata Hassk., Bonplandia 7: 181-183, 1859.

Laurentia longiflora (L.) Endl. var. muncinata (Hassk.) E.

Wimm., Nat. Hist. Mus. Wien, Ann. 56: 337, 1948.

446. Saxo-fridericia Schomb., Rapat. Frid.-Aug. Braunschw. 13, pl. 2, 1845.

447. Nostoc Paracelsus ex S. F. Gray, Nat. Arr. Brit. Pl. 1: 351, 1821.

Nostoch Paracelsus, Sämtliche Werke, ed. J. Strebel 8: 488, 1949.

Nostoc Vaucher, Histoire des conferves d'eau douce 203, 1803.

Nostoc Vaucher ex Born. \& Flah., Ann. Sci. Nat. Bot. VII, 7: 181-183, 1888.

Tremella Nostoc L., Sp. Pl., ed. 2, 1,625, 1763.

448. Phyllites kaiwakaensis McQueen, Roy. Soc. N. Z., Trans. 82(3): 676, pl. 27, fig. 6, 1954.

449. Notonia Wight \& Arn., Prodr. Fl. Penins. Ind. Orient. 1: 207-208, 1834.

Notonia DC., Guillemin's Archives de Bot. 2: 518, 1833. Johnia Wight \& Arn., Prodr. Fl. Penins. Ind. Orient. 1: 449, 1834. 
450. Pandanus spurius Rumph., Herb. Amb. 4: 142-143, pl. 75, 1750.

P. spurius (Martelli) Rumph, Webbia 4(1): 31, 1913.

P. spurius Miq., Anal. Bot. Ind. 2: 27, 1851.

P. spurius Kurz, Jour. Bot. Brit. For. 5: 129, 1867.

P. spurius Rumph. ex Martelli, Soc. Bot. Ital., Bul. 299, 1904; repr. 2, 1904.

451. Brachycome superspecies leptocarpa B. uliginosa G. L.

Davis, Linn. Soc. N. S. W., Proc. 79: 203-204, fig. 1-4, 1955.

452. Cercospora platyspora Ellis \& Holway, Jour. Mycol. 3: 16, 1887.

Fusicladium depressum v. Hoehnel, Ann. Mycol. 1: 531, 1903.

See Chupp, Monogr. Fung. Gen. Cercospora 578, (1953)= [1954].

453. Lophostigma Brongn., Ann. Sci. Nat. Bot. VI, 1: 286, 1875. Lophostigma (Brongn.) Warb., Engler's Pflanzenreich IV, 9: $44,71,1900$.

454. Eusabatia Griseb., Gen. et Sp. Gent. 120, 1839. Eusabatia (Griseb.) Blake, Rhodora 17: 56, 1915.

See Wilbur, Rhodora 57: 5, 1955.

455. Pandanus saipanensis Kanehira, Jour. Jap. Bot. (Shokubutsu

Kenkyu Zasshi) 12: 549, fig. 16-17, 1936.

$P$. saipanensis Kanehira, Bot. Mag. [Tokyo] 50: 521-522,

fig. 43-44, 1936.

456. Pithecellobium monoptemum Kostermans, New \& Crit.

Malaysian Pl. 2: 1-3, fig. 1, 1954; Reinwardtia 3: 1-3, fig. 1, 1954 .

457. Sida acuta Burm. f., Fl. Ind. 147, 1768.

Silagurium longifolia Rumph., Herb. Amboin. 6: 45-46, pl.

18, fig. 2, 1750.

T sjeru-parua Rheede, Hort. Malabar. 10: 105, pl. 53, 1686.

458. Tulasnella metallica Rick, Broteria Ciênc. Nat. 3: 169,

1934.

Ceratobasidium plumbeum Martin, Mycologia 31: 513. fig.

21-27, 1939. 
C. atratum (Bres.) Rogers ex Martin, Lloydia 4: 262, 1941.

Corticium atratum Bres., Hedwigia 35: 290, 1896.

See Univ. Iowa Nat. Hist. Stud. 17: 31, 1935;

Rogers \& Jackson, Farlowia 1: 272, 1943.

459. Asplenium Trich. dentatum L., Sp. P1. 1,080, 1753.

A. dentatum L., Syst. Nat., ed. 10, 2: 1,323, 1759.

A. barbadense Jenman, Gard. Chron. III, 15: 134, 1894.

460. Wikströmia Spreng., Svensk. Vet. Akad, Stockholm, Handl. III, 9: 167, 1821.

Wickstroemia Endl., Prodr. Fl. Norfolk 47, 1833.

Capura Blanco, Fl. Filip. 264 (error typ. 644), 1837.

Diplomorpha Meissn., K. Bayerische Bot. Gesell. Regensburg, Denkschr. 3: 289, 1841.

D. elongata recurva (Hbd.) Heller, Geol. \& Nat. Hist. Surv. Minn. 9: (Minn. Bot. Stud. 1:) 859-860, 1897.

Capura L., Mant. Alt. 149, 1771. 


\section{CHAPTER 5}

500. Vitex trifolia L. var. $\alpha$ trifoliolata Schauer in DC., Prodr.

11: 683, 1847.

V. trifolia L. a trifoliata Cham., Linnaea 7: 107, 1832.

See Lam, H. J., Verbenaceae of Malayan Archip. 182, 1919.

501. Scirpus lateralis Forsk., Fl. Aegypt. Arab. 15, 1775.

S. lateriflorus Gmel., Syst. Nat. of Linnaeus, ed. 13 reform. by Gmel., 2(1): 127, 1796.

S. lateralis Retz., Obs. Bot. 4: 12, 1786.

S. erecto-gracilis Hayata, Icon. P1. Formosa 6: 114, fig. 31, 1916.

Isolepis uninodis Delile, Fl. Aegypt. 8-9, pl. 6, fig. 1, 1812.

S. uninodis (Delile) Boiss., Fl. Orient. 5: 380, 1884.

S. uninodis (Delile) Boiss. ex Beetle, Am. Jour. Bot. 29:

656, 1942.

S. erectus Poir. in Lam., Encyc. Méth. Bot. 6: 761, 1804.

See Christensen, Dansk. Bot. Arkiv 4: 12, 1922.

Blake, S. T., Roy. Soc. Queensland, Proc. 62: 83-88, 1952.

502. Hoya esculenta (Rumph.) Tsiang, Sunyatsenia 3: 176, 1936. Sussuela esculentum Rumph., Herb. Amb. 5: 467-468, pl.

175, fig. 2, 1750.

Hoya diversifolia Bl., Bijd. Fl. Nederl. Ind. 1,064, 1825. $H$. orbiculata Wall. ex Wight, Contr. Bot. Ind. 36, 1834.

See Furtado, Gardens' Bul. 12: 372, 1949.

503. Hemerocallis Lilio Asphodelus L., Sp. PI. 324, 1753. H. flava L., Sp. Pl., ed. 2, 1: 462, 1763.

504. Pseudotsuga glaucescens (Roezl) Bailly, Rev. Hort. (Paris) 67: 88-90, col. pl., 1895.

Abies glaucescens Roezl, Cat. Grain. Conif. Mex. 18581859: 5, 1858; Linnaea 29: 330, 1858. 
Picea glaucescens (Roezl) Gordon, Pinetum 149, 1858. A. religiosa (HBK.) Schlecht. \& Cham. var. glaucescens

(Roezl) Carr., Traité Gén. Conif., nouv. ed. 274, 1867. Pseudotsuga taxifolia (Poir.) Britton ex Sudw. var. glauca (Beissn.) Schneid. forma glaucescens Schneid. in Silva Tarouca, Uns. Freil.-Nadelh. 269, 1913.

505. Gelidium samoense Reinbold forma lineare Setchell, Univ.

Calif. Publ. Bot. 12: 99, 1926.

$G$. samoense Reinbold forma minus Setchell, Carnegie

Inst. Wash., Publ. 341: 163, 1924.

See Taxon 3: 234, 1954; 4: 118, 1955.

506. Brugmansia arborea (Pers.) Steud., Nomencl., ed. 2, 230, 484, 1841.

507. Cystopteris montana (Lam.) Bernh. ex Desv., Prodr. 264, 1827.

Polypodium montanum Lam., Fl. Fr. 1: 23, 1778.

Cyathea montana (Lam.) Sm., Acad. Roy. Sci. Turin, Mem. 5:

417, 1793.

Aspidium montanum (Lam.) Sw. in Schrad. Jour. 1800(2):

42-43, 1801.

Athyrium montanum Spreng., Anleit. Kenntn. Gewächse 3:

143, 1804.

Filix montana Underw., Our Nat. Ferns, ed. 6, 119, 1900.

Filicula montana (Lam.) Farw., Am. Midl. Nat. 12: 252, 1931.

Filicula Sequier, Pl. Veron., Suppl. 3: 54-55, tab. 1, 1754.

508. Peniophora admirabilis Burt, Mo. Bot. Gard., Ann. 12: 304 , $(1925)=[1926]$.

See Rogers \& Jackson, Farlowia 1: 310, 1943.

509. Dubautia raillárdioides Hbd., Fl. Haw. Is. 224, 1888.

Railliardia Gaud., Voy. Freycinet, Uranie, Bot. 469, pl. 83, $(1826)=[1830]$.

Dubautia Railliardioides Hbd. emend. Sherff, Bishop Mus., Bul. 135: 107, 1935.

510. Asclepias syriaca L., Sp. Pl. 214, 1753. Apocynum syriacum Clus., Rar. Pl. Hist. 5: Ixxxviii, 1601. 
511. Heracleum maximum W. Bartr., Travels 344, 1791; ed. 2, 342, 1792; 342, 1794.

H. lanatum Michx., Fl. Bor.-Am. 1: 166, 1803.

See Fernald, Rhodora 46: 50, 1944.

Rickett, Rhodora 46: 389-391, 1944.

Merrill, Bartonia 23: 26, 1945.

512. Musa Troglodylarum L., Sp. Pl., ed. 2, 2: 1,478, 1763.

M. Troglodytarum L., Sp. Pl., ed. 3, 2: 1,478, 1764.

M. Uranoscopos Rumph., Herb. Amb. 5: 137, pl. 61, fig. 2, 1750.

M. Uranoscopus Colla, Accad. R. Sci. Torino, Mem. 25:

387-388, 1820; repr. 59-60, 1820.

M. Fehi Bert. ex Vieillard, Ann. Sci. Nat. Bot. IV, 16: 45, 1862.

M. Sapientum L. ssp. M. Troglodytarum (L.) L. ex Baker,

Ann. Bot. 7: 214-215, 1893.

M. paradisiaca L. ssp. troglodytarum (L.) Baker ex K.

Schum. in Engler, Pflanzenreich IV, 45: 21, 1900.

M. sapientum L. var. M. troglodytarum (L.) L. ex Anon.,

Kew Bul. 250, 1894; Kew Bul., Add. Ser. 6: 22, 1906.

513. Thelephora candida Schw., Naturf. Ges. Leipzig, Schr. 1:

110, 1822; Fr., Elench. Fung. 1: 189, 1828.

T. candidissima Schw., Am. Phil. Soc., Trans. II, 4: 167,

1832.

Aleurodiscus candidus (Schw.) Burt, Mo. Bot. Gard., Ann.

5: 188, 1918.

T. (sub Merisma) candida (Schw.) Fr., Syst. Orb. Veg. 82,

1825.

Tremellodendron (sub Merismate) candidum (Schw.) Atk.,

Jour. Mycol. 8: 106, 1902.

514. Shorea costata (Correa) J. S. Presl in Berchtold \& J. S.

Presl, Opřirozenosti rostlin 2: 66, 1825.

Pterigium costatum Corréa de Serra, Mus. Hist. Nat. Paris,

Ann. 8: 397-398, pl. 6, fig. 1, 1806.

Dryobalanops aromatica Gaertn., Fruct. 4: 49, pl. 186, 1805.

515. Heleocharis kasakstanica Zinserl. in Komarov \& Shischkin,

Fl. U.R.S.S. 3: 78, 1935. 
516. Banksia J. R. \& G. Forst., Char. Gen. 7-8, pl. 4, 1776. Pimelia Banks \& Soland. ex Gaertn., Fruct. 1: 186, 1788. Banksia L. f., Suppl. 15, 126, 1781. Banksea Koen. in Retz., Obs. 3: 75, 1783.

Banke sia Bruce, Trav. Disc. Source Nile, ed. 2, 7: 181, 1805.

Banksia Bruce, Trav. Disc. Source Nile, ed. 2, 8: pl. 2223, 1805.

Banksia Domb. ex DC., Prodr. 3: 83, 1828.

517. Rubus rosaefolius $\mathrm{Sm}$, Pl. Icon. Ined. Herb. Linn. 3: pl. 60, 1791.

$R$. rosifolius Sm. emend. Hochr., Candollea 6: 464, 1936. See Focke, Sp. Ruborum 153, 1910.

518. Sclerotium bifrons Ellis \& Everh., N. Am. Fung. No. 2,554, 1891.

S. bifrons Ellis \& Everh. in Sacc., Syll. Fung. 14: 1,169, 1899.

Sclerotinia bifrons Seaver \& Shope, Mycologia 22: 3, pl., 1930.

Sclerotinia bifrons Whetzel, Mycologia 32: 126, 1940. Sclerotinia confundens Whetzel, Mycologia 32: 126, 1940. Sclerotinia Whetzelii Seaver, Mycologia 32: 127, 1940. Ciborinia bifrons (Whetzel) Whetzel, Mycologia 37: 668, 1945.

Ciborinia confundens (Whetzel) Whetzel, Mycologia 37: 668, 1945.

Ciborinia Whetzelii (Seaver) Seaver, N. Am. Cup Fung. Inoper. 70, 1951.

Ciborinia bifrons (Ellis \& Everh.) Seaver, N. Am. Cup Fung. Inoper. 71, 1951.

519. Metrosideros collina (J. R. \& G. Forst.) Gray ssp. polymorpha (Gaud.) Rock var. imbricata Rock, Hawaii Board Agric. \& For., Div. For., Bot. Bul. 4: 49, pl. 17, 1917.

M. polymorpha Gaud. ssp. imbricata Rock ex Skottsb.,

Göteborg Bot. Trädgård, Meddel. 15: 407, 1944.

520. Morelotia gahniaeformis Gaud., Bot. Voy. Frey. Uranie 416, $(1826)=$ [1829]; Atlas pl. 28, 1826. 
Gahnia gahniaeformis (Gaud.) Heller, Minn. Bot. Stud. 1: 802, 1897.

Lampocarya Gaudichaudii Brongn. in Duperrey, Voy. Bot. 2: 166, 1829.

G. Gaudichaudii (Brongn.) Steud., Syn. Pl. Glum. 2: 164, 1855.

See Benl, Bot. Archiv 40: 151-257, 1940.

521. Apocynum fol. androsaemi L., Sp. Pl. 213, 1753. A. androsaemifolium L., Sp. Pl., ed. 2, 311, 1764. A. androsaemifol. L., Syst. Nat., ed. 12 reform. 2: 192, 1767.

A. androsaemi folium Mill., Gard. Dict., ed. 8, Apocynum No. 1, 1768.

A. androsaemifol. L., Syst. Nat., ed. 13, 2: 192, 1770. A. androsaemifolium L. Sp. Pl. of Linnaeus, ed. 4 by Willd., 1(2): 1,259, 1797. Apocinum androsaemi-f. Lestib., Botanogr. Belg., ed. 2, 3: 83, 1799.

Apocynum muscipulum Moench, Meth. Pl. Hort. Marburg 464, 1794.

522. Scirpus maritimus macrostachyus Michx., Fl. Bor.-Am. 1:

32, 1803.

S. macrostachyos Lam., Tabl. Encyc. et Méth. 1: 142, 1791. S. maritimus L. $\beta$ macrostachyus (Michx.) Pers., Syn. Pl. 1: 68,1805 .

Bolboschoenus macrostachyus (Willd.) Grossh., Fl.

Caucasus 1: 145, 1928.

S. maritimus L. var. paludosus (A. Nels.) Gleason, New

Britton \& Brown Ill. Fl. 1: 276, 1952.

S. robustus Pursh, Fl. Am. Sept. 56, 1814.

See Britton \& Brown, Ill. Fl. N. E. U. S., ed. 2, 1: 333, 1913.

Beetle, Am. Jour. Bot. 29: 84, 1942.

Fernald, Gray's Man. Bot., ed. 8, 272, 1950.

523. Arctium Lappa L., Sp. Pl. 816, 1753. A. Lappa L. ssp. majus (Bernh.) J. Arènes, Jard. Bot. État Bruxelles, Bul. 20(1): 75-77, 1950. A. majus Bernh., Syst. Verz. Pfl. Erf. 154, 1800. 
Lappa major Gaertn., Fruct. 2: 379, pl. 162, fig. 3, (1791)= [1802].

L. communis Coss. \& Germ. var. $\beta$ major (DC.) Coss. \& Germ., Fl. Env. Paris 389, 1845.

L. vulgaris Hill, Veg. Syst. 4: 28, pl. 25, fig. 1, 1762.

524. Nototrichium sandwicense (Gray) Hbd., var. Forbe sii Sherff, Bot. Leafl. 2: 2, 1950.

525. Tiarella cordifolia L. var. typica Lakela, Am. Jour. Bot. 24: 344-351, 1937.

T. cordifolia typica (Lakela) Wherry, Acad. Nat. Sci. Phila., Not. Nat. 42: 4, 1940.

526. Quesnelia Gaud., Bot. Voy. La Bonite, pl. 54, $(1844-1866)=$ [1842].

Guesmelia Gaud. ex Walp., Ann. Bot. Syst. 1: 841, 1849. Lievena Regel, Gartenfl. 29: 289, pl. 1,024, 1880.

527. Smelowskia ? Californica Gray, Am. Acad. Arts Sci., Proc. 6: 520, 1866.

528. Cladium P. Browne, Civ. Nat. Hist. Jamaica 114, 1756. Mariscus (Hall.) Zinn, Cat. Hort. Goett. 79, 1757.

See Sprague, Kew Bul. 218, 1934.

529. Clematis virginiana L. var. missouriensis (Rydb.?) Palmer

\& Steyerm., Mo. Bot. Gard., Ann. 22: 542, 1935.

C. Missouriensis Rydb. in Britton, Man. Fl. N. E. U. S. 421, 1901.

C. virginiana L. forma missouriensis (Rydb.) Fernald,

Rhodora 39: 309, 1937.

530. Phlox carolina heterophylla (Beauvais ex Brand) Wherry,

Bartonia 23: 6-7, 1945.

$P$. heterophylla Beauvais ex Brand, Engler Pflanzenreich IV, 250: 55, 1907.

531. Nymphaea L., Gen. Pl., ed. 5, 227, 1754; ed. 6, 264, 1764;

Sp. Pl. 510, 1753.

Castalia Salisb. in Koenig \& Sims, Ann. Bot. 2: 71-76,

$(1806)=[1805$, May 1].

Leuconymphaea Ludwig ex Ktze., Rev. Gen. 1: 11, 1891. Nymphea Raf., Med. Fl. \& Bot. U. S. 2: 44-45, 1830. 
Nymphozanthus L. C. Rich., Anal. du Fruit 63, 68, 103, 1808 May; Mus. Paris, Ann. 17: 230, 1811.

Nymphosanthus L. C. Rich., Anal. du Fruit 68, 1808 May;

Mus. Paris, Ann. 17: 230, 1811.

Nuphar Sm. in Sibth. \& Sm., Fl. Graec. Prodr. 1: 361-362, 1806.

Nenuphar Hayne, Getreue Darstell. u. Beschreib. Arzneik.

4: pl. 36, 1816.

Blephara Sm., Mem. \& Corr. 1: 576, 1832.

Ropalon Raf., New Fl. N. Am. 2: 17, 1836.

Nufar Wallr., Linnaea 14: 582, 1840; (repr. as Erster Beitr.

Fl. Hercyn.) 212, 1840.

Nymphona Bubani, Fl. Pyr. 3: 260, 1901.

Nelumbo Adans., Fam. PI. 2: 76, 1763.

Nelumbium Juss., Gen. 68, 1789.

532. Aplodiscus DC., Prodr. 5: 350, 1836.

Aplodiscus (DC.) Gray, Am. Acad. Arts Sci., Proc. 8: 638, 1873.

533. Nania polymorpha Gaud. var. glaberrima Lévl., Fedde

Repert. 10: 149, 1911.

Metrosideros collina (J. R. \& G. Forst.) Gray ssp. poly-

morpha (Gaud.) Rock var. glaberrima (Lévl.) Rock, Terr.

Hawaii, Board Agric. \& For., Div. For., Bot. Bul. 4:

69-71, pl. 29-30, 1917.

534. Aristeae B. \& H., Gen. PI. 3: 684, 1883.

Aristeinae Diels in Engler \& Prantl, Nat. Pflanzenfam., ed.

2, 15a: 480, 1930.

535. Camellia Thea Link, Enum. P1. Hort. Berol. 73, 1822. Thea sinensis L., Sp. P1. 515, 1753.

Camellia sinensis (L.) Ktze., Hort. Petrop., Acta 10: 195 in obs., 1887.

See Perrier de la Bathie, in Humbert, Fl. Madagascar, fam. 134: 11, 1951.

536. Schiedea salicaria Hbd., Fl. Haw. Is. 33, 1888.

S. Salicaria Hbd. var. typica Sherff, Am. Jour. Bot. 36: 499, 1949.

537. Ipomoea acetosaefolia (Vahl) Vahl ex R. \& S., Syst. Veg. of Linnaeus, ed. by R. \& S., 4: 246, 1819. 
Convolvulus acetosaefolius Vahl, Eclog. Am. 1: 18, 1796. C. littoralis L., Syst. Nat., ed. 10, 924, 1759.

C. stoloniferus Cyrill., Pl. Rar. Neap. 1: 14-17, pl. 5, 1788.

I. littoralis (L.) Boiss., Fl. Orient. 4: 112, 1879.

I. littoralis Bl., Bijdr. Fl. Nederl. Indië 713, 1825.

I. stolonifera (Cyrill.) Gmel., Syst. Nat. of Linnaeus, ed.

13 by Gmel., 2: 345, 1791.

I. carnosa R. Br., Prodr. Fl. Nov. Holl. 485, 1810.

See van Oostroom, Blumea 3: 540, 1940.

538. Boerhavia L., Gen. Pl., ed. 5, 4, 1754; Sp. Pl. 3, 1753.

539. Anetia Endl., Gen. P1. 923, 1839.

Anetium Splitgerber, Nat. Ges., Tiijds. 7: 395, 1840.

Pteridanetium Copel., Gen. Fil. 224, 1947.

See Morton, Am. Fern Jour. 43(2): 71, 1953.

540. Corallina barbata L. Syst. Nat., ed. 10, 1: 806, 1758. Cymopolia Barbata (L.) Lamx., Hist. Polyp. 293, 1816. Cymopolia Rosarium Lamx., Hist. Polyp. 294, 1816.

See Howe in Britton \& Millspaugh, Bahama Fl. 605, 1920.

541. Apiaceae Lindl., Nat. Syst., ed. 2, 21, 1836. Ammiaceae Presl, Delic. Prag. 1: 132, 1822. Umbelliferae Crantz, Class. Umbellif. 41, 1769.

542. Amida Nutt., Am. Phil. Soc., Trans. II, 7: 390, 1841.

543. Przewalskia Maxim., Acad. Imp. Sci. St.-Pétersb., Bul. 27: 507, 1881.

544. Cinchona L., Gen. Pl., ed. 5, 79, 1754; Sp. Pl. 172, 1753.

545. Eupritchardia Ktze., Rev. Gen. 3(2): 323, 1898.

Pritchardia Seem. \& Wendl. in H. Wendl., Bonplandia 10:

197, 1862; 9: 260, 1861.

Pritchardia Unger in Endl., Gen. Pl., Suppl. 2: 102, 1842. Pritchardioxylon Drude in Engler \& Prantl, Nat. Pflanzenfam., Nachtr. 50, 1897.

546. Draba Crockeri Lemmon, Torrey Bot. Club, Bul. 16: 221, 1889.

547. Acer barbatum Michx. var. Longii (Fern.) Fern. forma platylobum (Fern.) Fern., Rhodora 47: 160, 1945. A. floridanum (Chapm.) Pax var. Longii Fern. forma platylobum Fern., Rhodora 44: 426-428, pl. 727, 19 位. 
548. Pinus tuberculata sensu Gord., Hort. Soc. London, Jour. 4: 218-220, ill., 1849.

P. tuberculata D. Don, Linn. Soc. Lond., Trans. 17: 442, $(1837)=[1836]$.

$P$. attenuata Lemmon, Mining \& Sci. Press 64: 45, 1892,

Jan. 16; Lemmon in Sarg., Gard. \& For. 5: 65, 1892, Feb. 10.

549. Chonemorpha penangensis Ridley, Straits \& Fed. Malay

States, Agric. Bul. 10: 146-148, 1911.

550. Phaseolus acutifolius Gray, Pl. Wright. 1: 43-44, 1850;

Smithsonian Contr. Know. 3(5): 43-44, 1850.

551. Korthalsella striata Danser, Jard. Bot. Buitenzorg, Bul. III, 14(2): 124, 1937.

552. Plectritis californica (Suksd.) Dyal, Am. Midl. Nat. 42:

$$
\text { 495, } 1949 .
$$

Aligera californica Suksd., Werdenda 1(4): 44, 1927. A. macrocera (T. \& G.) Suksd., Deutsch. Bot. Monatschr. 15(5): 147, 1897.

Plectritis macrocera T. \& G., Fl. N. Am. 2: 50, 1841.

553. Lycopodium venustulum Gaud. var. heterophyllum (Hook.)

Hbd. ex Nessel, Die Bärlappgewächse 308, pl. 70, fig. 67, 1939.

L. heterophyllum Hook. \& Grev., Icon. Fil. pl. 113, 1831.

L. heterophyllum Grev. \& Hook., Bot. Mag. 2: 376, 1831.

554. Saintpierrea Germain de Saint Pierre, Journ. des Roses 39, in obs., 1878.

555. Phylanthera Noronha, Batav. Gen., Verh., ed. 1, 5(4): 3, 1790.

Phyllanthera Bl., Bijdr. Fl. Nederl. Indië 1,048, (1825)= [1827].

556. Tillaea L., Gen. Pl., ed. 5, 62, 1754.

557. Leveillea Vaniot, Acad. Intern. Géogr. Bot., Bul. 12: 29, 1903.

558. Luehea Willd., Ges. Naturf. Fr., Neue Schr. 3: 410, pl. 5, 1801.

Luhea Willd. ex DC., Prodr. 1: 517, 1824. 
559. Humbertia Lam., Encyc. Méth. Bot. 2: 356, 1786.

Humbertiella Hochr., Acad. Sci. Paris, Compt. Rendu 182: $1,485,1926$.

Humbertochloa A. Camus \& Stapf, Soc. Bot. France, Bul. 81: 467, 1934.

560. Eragrostis Hosakai Degener, Fl. Haw., fam. 47: April 16, 1937.

561. Crepis Froelichiana DC., Prodr. 7: 165, 1838.

562. Margyricarpus Ruiz \& Pavon, Fl. Peruv. Prodr. 7, pl. 33, 1794.

563. Alchemilla vulgaris L., Sp. Pl. 123, 1753. A. vulgaris agg., Clapham, Tutin \& Warburg, Fl. Brit. Is. 505, 1952.

564. Geigeria plumosa Muschl. ampl. Merxm., Bot. Staatssamml. München, Mitt. 7: 276-277, 1953.

565. Gynoxys Macfrancisci Cuatr., Fieldiana Bot. 27(2): 3-4, 1951.

566. Senecio Alberti-Smithii Cuatr., Fieldiana Bot. 27(2): 21-22, 1951.

567. Senecio Fortunatus Cuatr., Fieldiana Bot. 27(2): 47, 1951. 568. Heterosporium tshawytschae Doty \& Slater, Am. Midl. Nat. 36: 663-665, 1946.

569. Canavalia turgida Grah. in Wall., Cat. (Num. List Pl. E. Ind.) No. 5,539, 1832.

Lablab microcarpus DC., Prodr. 2: 402, 1825. C. microcarpa (DC.) Merr., Interp. Rumph. Herb. Amb. 280, 1917.

C. microcarpa (DC.) Piper, Biol. Soc. Wash., Proc. 30: 176, 1917.

570. Canarium vulgare Rumph., Herb. Amb. 2: 145-150, pl. 47, 1750; Stickm., Herb. Amb. 9, 1754; Stickm. in L., Amoen. Acad. 4: 121, 1759.

C. indicum Stickm. in L., Amoen. Acad. 4: 143, 1759. C. commune L., Mant. 127, 1767.

571. Aecidium Hyperici frondosi Schw., Naturf. Ges. Leipzig, Schr. 1: 68, 1822. 
Uredo hyperici Spreng., Syst. Veg. of Linnaeus, ed. 16 by Spreng. 4: 572, 1827.

Uromyces triquetmes Cooke, Portland Soc. Nat. Hist., Proc.

1: $184,1869$.

Uromyces Hyperici (Schw.) Curt., Geol. \& Nat. Hist. Surv.

N. Carol. Bot. 3: 123, 1867.

Nigredo Hyperici-frondosi (Schw.) Arth., Sci. Congr.

Internat. Bot. Wien, Résult. 344, 1906.

572. Conomorpha roraimae Steyerm., Fieldiana Bot. 28(3): 468, 1953.

573. Trochodendroxylon Beckii Hergert \& Phinney, Torrey Bot.

Club, Bul. 81: 118-122, fig. 1-4, 1954.

574. Labordia Fagraeoidea Gaud. var. $\epsilon$ sessilis (Gray) Sherff forma 1 glabrescens Sherff, Field Mus. Nat. Hist., Bot. Ser. 17(6): 481-482, 1939.

575. Sagittaria Hitchcochii Gdgr., Soc. Bot. France, Bul. 66: 294, (1919) $=$ [1920].

576. Avena Necker, Elem. Bot. 3: 217, 1790. Avena L., Gen. Pl., ed. 5, 34, 1754.

577. Litanum Nieuwl., Am. Midl. Nat. 4: 90, 1915.

578. Senecio Szyszylowiczii Hieron., Engler Bot. Jahrb. 36: 508-509, 1905.

579. Solanum Woahense Dunal in DC., Prodr. 13(1): 268, 1852. S. sandwicense H. \& A., Bot. Beechey Voy. 42, 1842. Carex wahuensis C. A. Meyer, Acad. St. Petersb., Mem. 1: 218, pl. 10, 1831.

Chenopodium oahuense (Meyen) Aellen, Ostenia 98-101, 1933.

Lepidium O-Waihiense C. \& S., Linnaea 1: 32, 1826. Eragrostis wahowensis Trin., Acad. St. Petersb., VI, Math. Phys. Nat. 1: 412, $(1830)=[1831]$.

580. Erica caffra L., Sp. Pl. 353, 1753.

581. Dictyocalyx Hook. f., Linn. Soc. Lond., Proc. 1: 277, 1849.

582. Cakile lanceolata (Willd.) O. E. Schulz, Engler Pflanzenreich IV, 105(2): 26-27, 1923.

C. edentula (Bigel.) Hook., Fl. Bor. Am. 1: 59, (1840)= [1830]. 
C. lanceolata (Willd.) O. E. Schulz in Urban, Symb. Antill. 3(3): 504, 1903.

583. Pleuropetalum Hook. f., Linn. Soc. Lond., Proc. 1: 278$279,(1849)=[1845]$.

Pleuropetalon Bl., Mus. Bot. Lugd.-Bat. 1: 248, 1850.

584. Prunus serotina Ehrh. var. eximia (Small) Little, Phytologia

4: 309-310, 1953.

P. eximia Small, Torreya 1: 146-147, 1901.

Padus eximia (Small) Small, Fl. S. E. U. S. 573-574, 1,331,

1903.

Prunus serotina Ehrh. ssp. eximia (Small) McVaugh, Brittonia 7: 302-303, 1951.

585. Betulinum hanenisiense Watari, Jap. Jour. Bot. 13: 503506, fig. 1-2, pl. 1A, 1948.

Betula hanenisiensis (Watari) Watari, Univ. Tokyo, Fac.

Sci. sect. 3, Bot., Jour. 6(1-3): 107, 1952.

586. Diplacorchis Summerh., Kew Bul. 379, 1931.

Brachycorythoides Summerh., Kew Bul. 379, 1931.

Eu-Diplacorchis Summerh., Kew Bul. 379, 1931.

Diplacorchis Schltr., Bot. Centralbl., Beih. 38(2): 127,

1921.

See Bullock, Taxon 3: 145, 1954.

587. Anemone ludoviciana Nutt., Gen. N. Am. Pl. 2: 20-21, 1818.

A. Nuttalliana A. P. DC., Reg. Veg. Syst. Nat. 193, 1818. Pulsatilla ludoviciana (Nutt.) Heller, Cat. N. Am. Pl., ed.

2, 80, 1900.

A. patens L. var. Nuttalliana (A. P. DC.) Gray, Man., ed.

5, 36, 1867.

A. patens L. var. Wolfgangiana (Bess.) Koch ex Hegi,

Illustr. Fl. Mitteleur. 3(1): 535, 1912.

P. patens (L.) Mill. var. Wolfgangiana (Bess.) Trautv. \&

Meyer in Midd., Reis. Ausserst. N. \& Ost Sibir. 1(2):

7, 1856.

P. hirsutissima (Pursh) Britton, New York Acad. Sci., Ann.

6: 217, 1891.

P. patens (L.) Mill., Gard. Dict., ed. 8, Pulsatilla No. 4, 1768.

See Löve, Svensk. Bot. Tidskr. 48: 219-222, 1954. 
588. Aerva Forsk., Fl. Aegypt. Arab. 170-171, 1775.

A. tomentosa Lam., Encyc. Méth. Bot. 1: 46, 1783.

589. Acacia ? tetragonocarpa Meisn. in Lehm., Pl. Preiss. 1(1): 4, 1844.

Tetracheilos Meisneri Lehm., Pl. Preiss. 2(2-3): 368, 389, 1848.

590. Pseudosolidum McG. ex Lloyd, Mycol. Writ. 7: 1,206, pl. 249, fig. 2,497, 1923.

Pseudosolidum Lloyd teste Stevenson \& Cash, Lloyd Libr. \& Mus., Bul. 35 (Mycol. Ser. 8): 22, 1936.

Hypoxylon solidum Berk. \& Curt., Acad. Nat. Sci. Phila.,

Jour. II, 2: 286, 1854.

Hypocreopsis solidus Lloyd ex Stevenson \& Cash, Lloyd

Libr. \& Mus., Bul. 35 (Mycol. Ser. 8): 22, fig. 2,497, 1936.

Rhytisma solidum Schw. ex Stevenson \& Cash, Lloyd Libr. \& Mus., Bul. (Mycol. Ser. 8): 22, 1936.

591. Pandanus aruensis Martelli var. contractus Martelli,

Engler's Bot. Jahrb. 49(1): 64-65, 1912.

$P$. aruensis Martelli, Webbia 4(2): 419-420, pl. 33, fig. 9,

1914.

$P$. aruensis Martelli forma contracta Martelli, Webbia 4(1):

6, 1913.

592. Echites puberula Nutt., Fraser's Cat. 1813; reprinted in

Pitconia 2: 117, 1890.

E. puberula Michx., Fl. Bor.-Am. 1: 120, 1803.

E. difformis Walt., Fl. Carol. 98, 1788.

Trachelospermum difforme (Walt.) Gray, Syn. Fl. N. Am. 2(1): 85, 1878.

Forsteronia difformis (Walt.) A. DC., Prodr. 8: 437-438,

1844.

Secondatia difformis (Walt.) B. \& H., Gen. Pl. 2: 723, 1873. Parechtites Thunbergii Gray, Am. Acad. Arts Sci., Mem. 6: 403, 1859.

See Shinners, Rhodora 57: 290-293, 1955;

Cronquist et al., Rhodora 58: 23-24, 1956;

Shinners, Rhodora 58: 281-289, 1956;

Graustein, Rhodora 58: 20-22, 1956. 
593. Torrey, J. \& Gray, A., Fl. N. Am. 1: 1838-1840.

594. $\times$ Prunygdalus Moldenke, Phytologia 5: 90, 1954. $\times$ P. $\times$ hybrida Moldenke, Phytologia 5: 90, 1954.

595. Volvariopsis Earlei Murr., Mycologia 3: 282, 1911.

V. Earleae Murr., N. Am. Fl. 10: 142, 1917.

596. Euphiladelphus S. Y. Hu, Jour. Arn. Arb. 35: 303, 305, 312, 1954; 36: 52, 1955.

597. Asplenium Trichomanes ramosum L., Sp. P1. 1,083, 1753; ed. 2, 2: 1,541, 1763; ed. 3, 2: 1,541, 1764; Syst. Nat., ed. 13, 2: 690, 1770 .

A. viridi Huds., Fl. Angl. 385-386, 1762.

A. viride Huds. emend. Sw., Syn. Fil. 80, 1806.

598. Nardia S. F. Gray emend. Carrington, Brit. Hepat. 10, 1875. Nardius S. F. Gray, Nat. Arr. Brit. P1. 1: 679, 694, 1821. Narda Vell., Fl. Flum. 108, 1825; 3: pl. 24, 1827. Nardus L. Gen. Pl., ed. 5, 27, 1754. 


\section{CHAPTER 6}

600. Discovium Raf., Jour. Phys. Chim. Hist. Nat. 89: 96, 1819. Lesquerella S. Wats., Am. Acad. Arts Sci., Proc. 23: 249255, 1888.

601. Equisetum maximum Lam., Fl. France 1: 7, 1778.

E. Telemateia Ehrh., Hannovers Mag. 287, 1783; Naturk.

Wissensch., Beitr. 2: 159, 1788.

See Milde, Acad. Caes. Leop.-Carol. Germ. Nat. Curios., Nov. Acta 24(2): 19, 1865.

Fernald, Rhodora 49: 206, 1947.

602. Mariscus phleoides Nees ex Steud., Syn. P1. Glum. 2: 62, 1855; Nees, Linnaea 9: 286, 1835.

Cyperus phleoides (Nees ex Steud.) Mann, Am. Acad. Arts Sci., Proc. 7: 208, 1867.

Cyperus (Mariscus) phleoides Nees ex Steud., Hbd., Fl. Haw. Is. 469, 1888.

603. Ochrosia parviflora Henslow, Ann. Nat. Hist. or Mag. Zool. Bot. Geol. 1: 345-346, 1838.

Cerbera parviflora Forst. f., Flor. Ins. Austral. Prodr. 19, 1786.

O. elliptica Labill., Sert. Austro-Caled. 25, pl. 30, 18241825.

O. noumeensis Baill. ex Guillaumin, Mus. Col. Marseille, Ann. 19: 34, 1911.

O. borbonica J. F. Gmel., Syst. Nat. of Linnaeus, ed. 13 by Gmel., 2(1): 439, 1791.

O. maculata Jacq., Icon. Rar. 2: pl. 321, 1786-1793.

Cerbera maculata (Jacq.) Willd., Sp. Pl. of Linnaeus, ed. 4 by Willd., 1(2): 1,223, 1797.

C. parviflora Wallich, Cat. (Num. List Pl. E. Ind.) No. 1,584, 1828. 
604. Acer saccharum Marsh., Arbustr. Am. 4, 1785. A. sacchatum Mill., Gard. Dict., abr. ed. 6, Acer No. 6, 1771.

A. saccharophorum K. Koch, Hort. Dendrol. 80, 1853. A. Treleaseanum Bush, Am. Midl. Nat. 12: 502, 1931.

See Mackenzie, Rhodora 28: 111, 1926; 28: 233, 1926. Sudworth, Rhodora 28: 179, 1926; Bot. Gaz. 100: 312, 1938.

Sprague, Kew. Bul. 81-82, 1929.

Bush, Am. Midl. Nat. 15: 784, 1934.

Rousseau, Inst. Bot. Univ. Montreal, Contr. 35: 1-66, 1940; 42: 17, 1947.

Gleason, Phytologia 2: 206-209, 1947.

605. Lithocarpus Rodgerianus A. Camus, Mus. Hist. Nat. Paris, Bul. II, 4: 913, 1931.

Pasania Rodgeriana A. Camus, Mus. Hist. Nat. Paris, Bul. II, 3: 690, 1931.

606. Dryopteris simulata Davenp., Bot. Gaz. 19: 497, 1894. Aspidium simulatum Davenp., Bot. Gaz. 19: 495-496, 1894. Nephrodium simulatum Davenp., Bot. Gaz. 19: 497, 1894. Lastrea simulata Davenp., Bot. Gaz. 19: 497, 1894. Thelypteris simulata (Davenp.) Nieuwl., Am. Midl. Nat. 1: 226, 1910.

607. Mammea J. Agardh, Symb. 1: 22, 1841; Linnaea 15: 22, 1841.

Mammea L., Gen. Pl., ed. 5, 228, 1754.

608. Amaranthus L., Gen. Pl., ed. 5, 427, 1754; Sp. Pl. 989-991, 1753.

Amarantus L., Internat. Code Bot. Nomencl. Stockholm. 44, 1952.

609. Diodia teres Walt., Fl. Carol. 87, 1788. Spermacoce diodina Michx., Fl. Bor.-Am. 1: 82, 1803.

See Blake, Rhodora 17: 129, 1915.

Fernald \& Griscom, Rhodora 39: 306, 1937.

610. Barringtonia J. R. \& G. Forst., Char. Gen. 75-76, pl. 38, 1776. 
Butonica Rumph., Herb. Amb. 3: 179-180, pl. 114, 1750. Agasta Miers, Linn. Soc. Lond., Trans. Bot. II, 1: 59, 1875. Baranda Llanos, Acad. Cienc. Madrid, Mem. 2: 502, 1859. Botryoropis Presl, Bohem. Ges., Abh. 6: 580-581, 1851; repr. as Epim. Bot. 220, 1849.

Butonica Juss., Gen. Pl. 326, 1789.

Doxomma Miers, Linn. Soc. Lond., Trans. Bot. II, 1: 98$107,1875$.

Megadendron Miers, Linn. Soc. Lond., Trans. Bot. II, 1: 109-111, 1875.

Menichea Sonner., Voy. N. Guin. 133, pl. 92-93, 1776. Meteorus Lour., Fl. Cochinch. 410, 1790.

Mitraria J. F. Gmel., Syst. Nat. of Linnaeus, ed. 13 by Gmel., 799, 1791.

Stravadium Juss., Gen. 326, 1789.

611. NEO-URBANIA Fawc. \& Rendle, Jour. Bot. Bric. For. 47: 125, 1909.

612. Cyathodes imbricata Stschegleew, Soc. Imp. Nat. Mosc., Bul. 32 (1): 10, 1859.

Styphelia Grayana Rock, Ind. Trees Haw. Is. 366, 1913. S. Douglasii (Gray) F. Muell. in Skottsb., Hort. Gotoburg, Acta 2: 255, 1926.

S. Douglasii (Gray) Hochr., Candollea 6: 470, 1936.

613. Schiedea Gregoriana Degener, Fl. Haw. 119: 4/9/36. S. kealiae Caum \& Hosaka, Bishop Mus., Occas. Pap. 11 (23): 3-5, 1936, April 10.

614. Barbosella crassifolia (Edw.) Schltr. var. minor Hoehne, Orquidea 11(1): 32, pl. 2, fig. 1, 1948.

615. Coprosma J. R. \& G. Forst., Char. Gen. 137-138, pl. 69, 1776.

Euarthronia Nutt. in Gray, Am. Acad. Arts. Sci., Proc. 4: 49, 1860.

Eurynome DC., Prodr. 4: 475, 1830.

Marquisia A. Rich., Soc. Hist. Nat. Paris, Mém. 5: 192, $(1834)=[1830]$.

Pelaphia Banks \& Soland. ex A. Cunn., Ann. Nat. Hist. 2: 206, 1839. 
616. Cestrum Weberbauerei Francey, Candollea 7: 5-6, 1936.

See Candollea 7: 509-517, 1938.

617. Lobelia affinis Wall., Cat. (Num. List. Pl. E. Ind.), No. 1,311, 1828; DC., Prodr. 7(2): 360, 1839.

L. affinis Wall. ex Hook. f. \& Thomps., Fl. Brit. Ind. 3:

424, 1882.

L. zeylanica L., Sp. Pl. 1,323, 1753.

L. succulenta Bl., Bijd. Fl. Nederl. Ind. 728, 1825.

618. Eclipta alba (L.) Hassk., Pl. Jav. Rarior. 528, 1848.

Verbesina alba L., Sp. P1. 902, 1753.

$V$. prostrata L., Sp. Pl. 902, 1753.

Cotula alba (L.) L., Syst. Nat., ed. 12, 2: 564, 1767.

Eclipta prostrata (L.) L., Mant. P1. Alt. 286, 1771.

E. erecta L., Mant. Pl. Alt. 286, 1771.

See Exell, Cat. Vasc. Pl. S. Tomé 225, 1944.

Fernald, Rhodora 47: 196-197, 1945.

Cronquist, Rhodora 47: 398-399, 1945.

619. Chamaecrista Chamaecrista (L.) Britton, Torrey Bot. Club,

Bul. 44: 12, 1917.

Cassia Chamaecrista L., Sp. Pl. 379, 1753.

Chamaecrista Pavonis Cass. ex Greene, Pittonia 3: 241,

1897.

620. Salacia L., Mant. P1. Alt. 2: 159-160, 293, 1771.

Salacia Pant., Beitr. Kenntn. Foss. Bacill. Ung., ed. 2,

2: 69-70, 1903.

621. Malus ioensis (Wood) Britton in Britton \& Brown, Ill. Fl. N.

States \& Can. 2: 235, 1897.

Pyrus coronaria L. $\beta$ Ioensis Wood, Class-book Bot., rev. ed., 334, 1861.

Pyrus Ioensis (Wood) Bailey, Am. Garden 12: 473-474, fig。 7-8, 1891.

622. Dilphinium peregrinum L., Sp. Pl. 531, 1753.

623. Herpophyllon Farlow in De Toni, Syll. Alg. 4(4): 1,713, 1902.

Herpophyllum J. Agardh, Analecta Alg。Cont. 2: 62-63, 1894. 
624. Picria Lour., Fl. Cochinch.393, 1790; ed. by Willd., 478, 1793.

See Merrill, Am. Phil. Soc., Trans. 24 (2): 29, 32, 352, 1935.

625. Nectria ditissima Tulasne, Sel. Fung. Carp. 72, pl. 13, fig. $1-4,1865$.

Sphaeria decidua Tode, Fungi Meckl. 2: 31, 1791.

S. coccinea Pers., Icon. \& Descr. Fung. 2: 47, pl. 12, fig. 2,a, b, c, 1800; Syn. Fung. 49-50, 1801.

N. coccinea (Pers.) Fr., Summa Veg. Scand. 387, 1849.

Creonectria coccinea (Pers.) Seaver, Mycologia 1: 188-189, 1909.

See Ashcroft, W. Va. Agric. Exp. Sta., Bul. 261: 27-42, 1934.

Petch, Brit. Mycol. Soc. 21: 243-301, 1938.

Lohman, Lloydia 6: 98, 1943.

626. Myroxylon L. f., Suppl. 34, 1781.

Myroxylon J.R. \& G. Forst., Char. Gen. 125, 1776.

Toluifera L., Sp. Pl. 384, 1753.

Xylosma Forst. f., Flor Ins. Austral. Prodr. 72, 1786.

627. Pandanus Ala-Kai Martelli, Univ. Calif., Publ. Bot. 17: 174, pl. 24, 1933.

628. Neyraudia Hook. f., Fl. Brit. Ind. 7: 305, 1896.

Neraudia Gaud., Bot. Voy. Freyc. Uranie 500, (1826)= [1830].

629. Phajus Tankervillii (Banks in L'Her.) Bl., Mus. Bot. Lugd.Bat. 2: 177, 1856.

Limodorum Tankervilliae Willd., Sp. Pl. of Linnaeus, ed. 4 by Willd., 4: 122, 1805.

L. Tancarvilleae L'Her., Sert. Angl. 28, 1788.

L. Incarvillei Pers., Syn. Pl. 2: 520, 1807.

Bletia Tankervilliae R. Br., Curtis's Bot. Mag. 44: pl. $1,924,1817$.

See Merrill, Am. Phil. Soc., Trans. n. s. 24(2): 123, 1935. Hawkes, Brittonia 7: 180, 1951.

630. Trisetum flavescens Beauv., ssp. I. T. pratense (Pers.)

Rouy, Fl. France 14: 140, 1913. 
631. Sanicula canadensis L. var. genuina Fern., Rhodora 42: 467-470, 1940.

S. canadensis L. var. typica H. Wolff, Engler's Pflanzenreich IV, 228: 67, 1913.

632. Vincentia angustifolia Gaud., Bot. Voy. Freyc. Uranie 417, $(1826)=[1829]$.

Cladium angustifolium (Gaud.) B. \& H., Gen. Pl. 3(2): 1,065-1,066, 1883.

C. angustifolium B. \& H. ex Drake, Ill. Fl. Ins. Mar. Pacif. 334, 1892.

633. Premna Taitensis Schauer in DC., Prodr. 11: 638, 1847. P. tahitensis DC. emend. F. Br., Bishop Mus., Bul. 130: 3, 248, 1935.

Scrophularioides arborea Forst. f., Flor. Ins. Austral. Prodr. $91,1786$.

$P$. arborea Farw., Druggists Circ. 63: 50, 1919.

$P$. arborea Roth, Nov. Pl. Sp. 287, 1821.

Gmelina arborea Roxb. ex Spreng., in Syst. Veg. of Linnaeus, ed. 16 by Spreng., 2: 765, 1825 .

634. Weinmannia borneensis Engler, Nat. Pflanzenfam., ed. 2, 18A: 255-256, 1930.

See Heine, Bot. Staatssamml. München, Mitt. 6: 212-213, 1953.

635. Elatostemma Pedunculatum J. R. \& G. Forst., Char. Gen. 105-106, pl. 53, 1776.

Langeveldia acuminata (Poir.) Gaud., Bot. Voy. Freyc. Uranie 494-495, $(1826)=[1830]$.

Procris acuminata Poir., in Lam. Encyc. Méth Bot. 5: 629, 1804.

636. Gonolobus jamaicensis Rendle, Jour. Bot. Brit. For. 74: 345, 1936.

G. rostratus R. Br., Wern. Soc., Mem. 1: 35, 1809.

G. rostratus sensu Griseb., Flora W. I. 142, 1864.

G. rostratus sensu Schlecht. in Urb., Symb. Antill. 1: 283, 1899.

See Furtado, Gardens' Bul. 12: 331, 1949. 
637. Lantana arubensis Moldenke, Some New Sp. \& Var. Verbenaceae 15-16, n. d.

638. Elymus condensatus pubens Piper, Erythea 7: 101, 1899. E. condensatus Presl var. pubens Piper ex Hitchc., U. S.

Dept. Agric., Misc. Publ. 200: 253, 843, 1935.

E. cinereus (Scribn.) Merr., Torrey Bot. Club, Bul. 29: 467, 1902.

639. Phaseolus cylindricus Stickm. in L., Amoen. Acad. 5: 132, 1759.

Vigna cylindrica (L.) Skeels, U. S. Dept. Agric., Bur. Pl. Ind., Bul. 282: 32, 1913.

Dolichos catjang Burm. f., Fl. Ind. 161, 1768.

Vigna Catjang (Burm. f.) Walp., Linnaea 13: 533, 1839.

See Merrill, Interp. Rumph. Herb. Amb. 284, 1917.

Lawrence, Gent. Herb. 8(1): 44, 1949.

640. Vernonia Baldwini Torr., Lyc. New York, Ann. 2: 211, 1828. $V$. interior Small, Torrey Bot. Club, Bul. 27: 279-280, 1900.

V. Baldwini interior Gleason, New York Bot. Gard., Bul. 4:

153, 1906.

V. interior Small var. Baldwini (Torr.) Mackenzie \& Bush, Man. Fl. Jackson Co. Mo., 190, 1902.

V. Baldwini Torr. var. interior (Small) Schubert, Rhodora 38: 370, 1936.

641. Lycopus uniflorus Michx., Fl. Bor.-Am. 1: 14, 1803.

See Fernald, Rhodora 36: 23-24, 1934.

642. Rubus alceaefolius Poir., Lam. Encyc. Méth Bot. 6: 247, 1804.

R. alceifolius Poir. emend. Hochr., Candollea 6: 461, 1936.

643. Andrzeiowskia Reichb., Icon. Pl. Crit. 1: 15, pl. 13, 1823. Andreoskia DC., Prodr. 1: 190, 1824.

644. Vinca rosea L., Syst. Nat., ed. 10, 2: 944, 1759. Lochnera rosea (L.) Reichenb., Consp. Regn. Veg. 134, 1828.

Catharanthus roseus (L.) G. Don, Gen. Syst. 4: 95, 1836. C. roseus (L.) G. Don var. albus (Sweet) Sweet ex Neal, In Gard. Hawaii 607, 1948. 
645. Calamagrostis ophitidis (Th. Howell) Nygren, Hereditas 40: 388, fig. 85-87, 1954.

646. Athyrium angustipinna Holttum, Singapore Gardens' Bul. 14: 8, 1953.

647. Lepidium lasiocarpum Nutt. ex T. \& G., F1. N. Am. 1: 115, 1838.

648. Cotinus obovatus Raf., Autikon Botanikon 82, 1840. C. americanus Nutt., N. Am. Sylva 3: 1, pl. 81, 1849.

See Little, Okla. Acad. Sci., Proc. 23: 21-23, 1943.

649. Sphaeria glomus Berk. \& Curt., N. Am. Fungi, No. 957,

n. d.; Berk. \& Curt. ex Berk., Grevillea 4: 152, 1876. Ophiolobus ? glomus (Berk. \& Curt.) Sacc., Syll. Fung. 2: 347, 1883; ex Underw. \& Earle, Alabama Agric. Exp. Sta., Bull. 80: 192, 1897.

Raphidospora glomus (Berk. \& Curt.) Cke, Grevillea 18: 16, 1889.

650. Mikania ( $\S$ Thyrsigerae) stereolepis B. L. Robins., Gray Herb., Contr. 104: 44-45, 1934.

651. Menthaceae Piper, U. S. Natl. Herb., Contr. 11: 486, 1906. Labiatae B. Juss., Hort. Trian., ordo 34, lxiii-1xx, 1759;

B. Juss. ex A. L. Juss., Gen. Pl. Ixiii-lxx, 1789.

Lamiaceae Lindl, Nat. Syst. Bot. Veg. Kingd. 275, 1836. Labiaceae Necker, Theod. Palat., Act. 2: 473, 1770.

652. Cercospora morina Chupp, Monogr. Fung. Gen. Cercospora 400, $(1953)=[1954]$.

C. moricola Cooke, Grevillea 12: 30, 1883.

C. mori Hara, Sericultural Assoc. Japan, Jour. 27 (314): 227

(Arabic 19), 1918.

C. mori Marchal \& Steyaert, Soc. Roy. Bot. Belg., Bull. 61: 166, 1929.

653. Ceramium affine Setch. \& Gardn. var. originale Dawson, Farlowia 4: 133, 1950.

654. Dicarphus rubens Raf., Med. Repos. II, 3: 423, 1806; II, 5:

355, 1808; Jour. Bot. (Paris) 2: 176, 1809; Specch.

Sci. 1: 194, 1814; Anal. Nat. Tabl. Univ. 211, 1815;

repr. in Lloyd, Mycol. Notes 1 (13) 129, 1903. 
See Gerard, Torrey Bot. Club, Bul. 12: 37, 1885; Merrill, Farlowia 1: 257, 1943.

655. Spegazzinia Backeb., Kakteenfreund, 2(10): 117, 1933.

656. Aërides Lour., Fl. Cochinch. 525, 1790.

657. Jarava Ruiz \& Pavon, Fl. Peruv. Prodr. 2, pl. 1, 1794.

Stipa L., Gen. Pl., ed. 5, 14, 1754; Sp.Pl. 78, 1753.

658. Fevillea L., Gen. Pl., ed. 5, 443, 1754; Sp. Pl. 1,013, 1753.

659. Aconitum kunasilense Nakai, Natl. Sci. Mus. (Tokyo), Bul. 32: 21, 1953.

660. Phleum pratense L. ssp. vulgare (C̈el.) Asch. \& Graebn. emend. Hyl., Bot. Notis. 357, 1953.

P. pratense L., ssp. A P. vulgare (C̈el.) Aschers.\&

Graebn., Syn. Mitteleur. F1. 2(7): 141-142, 1899.

P. pratense L. a vulgare Celakovsky, Prodr. Fl. Böhm. 38, 1867.

$P$. pratense L. s. str. Sterner ap. Lindman, Svensk Fanerogamfl., ed. 2, 74, 1926.

661. Pteronevron Fée, Gen. Fil, in Mém. Fam. Foug. 5: 204,

320 , pl. 25, B, fig. 1, $(1850-1852)=[1852]$.

See Pichi-Sermolli, Webbia 9: 361-362, 1953.

Rickett, Taxon 4: 185-188, 1955.

662. Senecio Danielis Cuatr., Fieldiana Bot. 27(2): 31, 1951.

663. Avena fatua L. ampl. Hausskn. ssp. eufatua Hyl., Bot.

Not is 355, 1953.

A. fatua L. ssp. fatua Thell., Naturf. Ges. Zürich, Vierteljahrsschr. 56: 319-320, 1911.

A. fatua L., Sp. Pl. 80, 1753.

664. Solanum incompletum Dunal in DC., Prodr. 13(1): 311, 1852.

665. Artemisia columbiensis Nutt., Gen. N. Am. Pl. 2: 142,

1818.

A. cana Pursh, Fl. Am. Sept. 2: 521, 1814.

See Ward, G. H., Dudley Herb., Contr. 4(6): 156, 188, 1953.

666. Cuscuta africana Willd., Sp. Pl. of Linnaeus, ed. 4 by Willd., 1(2): 703, 1797. 
Schrebera Schinoides L., Sp. Pl., ed. 3, 1,662, 1764.

Schinus myricoides L., Sp. P1. 388, 1753.

667. Gouldia terminalis (H. \& A.) Hbd. var. kaala Fosb. forma eukaala Fosb., Bishop Mus., Bul. 147: 49, 1937.

668. Gouldia terminalis (H. \& A.) Hbd. var. Hosakai Fosb., Bishop Mus., Bul. 147: 38, 1937.

669. Acanthonitschkea Speg., Buenos Aires Mus. Nac., Ann. III, 10: 116, 1908.

670. Macraea Lindl., Quart. Jour. Sci. Lit. \& Art 25: 104, 1828. Macraea Hook. f., Linn. Soc. Lond., Proc. 1: 278, 1849. Macraea Wight, Icon. 5: 27, 1852.

671. Jeffreya Shastensis Oreg. Comm. [Rept. 8]: third Page, [1853].

672. Elaphoglossum reticulatum Gaud., Bot. Voy. La Bonite, Atlas pl. 79, fig. 1-4, 1844.

673. Crucita hispanica L., Sp. Pl., ed. 2, 179, 1762.

Cruzita hispanica L., Syst. Nat., ed. 12, 129, 1767.

674. Rumex Britannica L., Sp. P1. 334, 1753.

675. Desmocephalum Hook. f., Linn. Soc. Lond., Proc. 1: 277278, 1849.

676. Bostrychia harveyi Mont. ecad distans (Harv.) De Berg,

Farlowia 3(4): 500-502, fig. 2, 1949.

B. distans ? Harv. in Hook. f., (Fl. Tasmania), Bot. Ant-

arctic Voy. Erebus \& Terror 5: 299, 1860.

B. distans Harv. in Hook. f., (Fl. N. Zeal. 2:), Bot. Ant-

arctic Voy. Erebus \& Terror 4: 226, 1855.

See Silva, Univ. Calif., Publ. Bot. 25: 269, 1952.

677. Ceanothus Americanus (L.) T. \& G., Fl. N. Am. 1: 264, $(1838-1840)=[1838]$.

678. Quercinum anataiense Watari, Jap. Jour. Bot. 11: 399-403, fig. 4-5, photo 2, D-G, 1941.

Quercus anataiensis (Watari) Watari, Univ. Tokyo, Fac.

Sci., Jour., Ser. III, Bot. 6(1-3): 107, 1952.

679. Pentaglossum linifolium Forsk., F1. Aegypt. Arab. 11, 1775. 
680. Secula viscidula (Michx.) Small, Fl. Miami 90, 200, 1913. Aeschynomene viscidula Michx. Fl. Bor.-Am. 2: 74-75, 1803.

681. Kyrtandra Gmel., Syst. Nat. of L., ed. 13 by Gmel., 2: 37, 1791.

Cyrtandra Juss., Gen. Pl. 121, 1789.

Kyrtandra aristata Blanco, Fl. Filip. 18, 1837.

682. Roxburghia Roxb., Pl. Coast Corom. 1: 29, pl. 32, 1795. Roxburghia Koenig ex Carey, Roxb., Fl. Ind., ed. Carey, 1: $168,1820$.

Roxburghia Koenig ex Carey, Roxb., Fl. Ind., ed. Carey, 3: 34, 1832.

See Rafinesque's assertion that Roxburgh names $R o x$ burghia for himself, Raf., F1. Tellur. 1: 17, $(1836)=[1837] ; 2(1): 96,(1836)=[1837]$.

683. Boehmeria Jacq., Enum. Pl. Carib. 9, 31, 1760.

684. Rhynchospora spicaeformis Hbd., Fl. Haw. Is. 477, 1888. R. spiciformis Hbd. emend. Skottsb., Hort. Gotob., Acta 15: $321,1944$.

685. Chaerophyllum Tainturieri Hook. var. floridanum C. \& R., U. S. Natl. Herb., Contr. 7: 60, 1900.

686. Clermontia Kakeana Meyen, Reise um die Erde 2: 358, 1834.

687. Cambogia G. Gutta L., Gen. Pl., ed. 5, addenda, 1754. C. Gutta L., Sp. Pl., ed. 2, 728, 1762; ed. 3, 728, 1764. Garcinia cambogia Desr. in Lam., Encyc. Méth. Bot. 3:

$701,(1789)=[1791]$.

688. Hippocratea Urceolus Tul. S. sp. xerophila H. Perr., Mus. Col. Marseille, Ann. V, 10: 27, 1942. 


\section{CHAPTER 7}

700. Santolina ChamaeCyparissus L., Sp. Pl. 842, 1753.

S. Chamae Cyparissus L., Sp. Pl., ed. 3, 2: 1,179, 1764.

Santolina Chamaecyparissus L. ex Hegi, Fl. Mitteleur. 6(2): 672, 1929.

S. chamaecyparissus L. ex Neal, In Gardens of Hawaii 749, 1949.

701. Lycopodium ecuadoricum Herter, Engler's Bot. Jahrb. 34: 48, 1909.

Urostachys Rolandii-principes Herter ex Nessel, Die Bärlappgewächse 117, 1939.

U. rolandi-principis Herter ex Nessel emend. Herter, Index Lycop. 79, 1949.

702. Limnanthes gracilis Howell, Fl. N. W. Am. 1: 108, 1897. L. gracilis Howell var. gracilis, Mason, Univ. Calif., Publ. Bot. 25(6): 487, 1952.

703. Pteridium Gleditsch in Scop., Fl. Carn. 169, 1760. Pteridium (Kuetz.) J. Agardh, Sp. Gen. Ord. Alg. 3(3): 218, 1898; 4(1): 710-716, 1897.

704. Ficus benghalensis L., Sp. Pl. 1,059, 1753; ed. 3, 1,514, 1864.

F. bengalensis L. ex Barrett, Torrey Bot. Club, Bul. 72: 394, 1945.

705. Agatea Gray, U. S. Expl. Exped. Bot. 15: 89-92, 1854. agataea Cass., Soc. Philom. Paris, Bul. Sci. 175, 1815. Agati Adans., Fam. Pl. 2: 326, 1763. Agation Brongn., Soc. Bot. France, Bul. 8: 79, 1861. Agathis Salisb., Linn. Soc. Lond., Trans. 8: 311-312, pl. 15, 1807. Agasta Miers, Linn. Soc. Lond. Bot., Trans. II, 1: 59, 1875. 
706. Astragalus dispermus dispermus James, Dudley Herb., Contr. 4(4): 67-68, 1951.

A. dispermus Gray, Am. Acad. Arts Sci., Proc. 13: 365, 1878.

707. Taxodium Washingtonium Winslow, Calif. Farmer (San Francisco newspaper) 2: 58, Aug. 24, 1854.

See St. John \& Krauss, Pacif. Sci. 8: 341-358, 1954.

Rousseau, Taxon 4: 40-42, 1955.

708. Stewartia montana, fol. ovatis acuminatis, W. Bartr., Travels N. Car. Florida 334, 1791.

See Merrill, Bartonia 23: 12, 1945.

709. Ficus Beniamina L., Mant. 129, 1767.

F. benjamina L., Syst. Nat., ed. 13, 2: 681, 1770.

F. benjamina L. var. nuda (Miq.) Barrett, Am. Midl. Nat.

45: 127-128, 1951.

Urostigma nudum Miq., Hooker's Lond. Jour. Bot. 6: 584, 1847.

U. benjamineum var. nuda (Miq.) Miq., Pl. Jungh. 1: 50, 1853.

Ficus nuda (Miq.) Miq., Mus. Bot. Lugd. Bat., Ann. 3: 267, 1867.

710. Boldu Adans., Fam. Pl. 2: 446, 1763.

Boldus Adans. emend. Schult. f. in R. \& S., Syst. Veg. of

Linnaeus, ed. nova by R. \& S., 7: p. X, 56, 1829.

711. Lobelia Gaudichaudii DC., Prodr. 7: 384, 1838.

L. Gaudichaudii DC. var. coccinea Rock, Torrey Bot. Club, Bul. 44: 238, pl. 15, 56, 57, 1917.

L. Gaudichaudii DC. var. typica St. John \& Hosaka, Bishop Mus., Occas. Pap. 14(8): 118-120, 1938.

712. Cercocarpus betuloides Nutt. ex T. \& G., Fl. N. Am. 1:

$427,1840$.

C. alnifolius Rydb., N. Am. Fl. 22: 421, 1913.

C. betulaefolius Nutt. var. Blancheae Schneid., Deut.

Dendrol. Gesell., Mitt. 14: 127, 1905.

C. betuloides Nutt. var. alnifolius (Rydb.) Dunkle, S. Calif. Acad. Sci., Bul. 39: 2, 1940.

C. montanus Raf. var. blanchae (Schneid.) F. L. Martin, Brittonia 7: 103, 1950. 
C. betuloides Nutt. var. blanchae (Schneid.) Little,

Phytologia 4: 308, 1953.

C. montanus Raf. var. glaber (S. Wats.) F. L. Martin, Brittonia 7: 101-102, 1950.

713. Paeonia lactiflora Pall., Reise Russ. Reiche 3: 286, 1776. P. albiflora Pall., Fl. Ross. 1(2): 92, 1788.

Poeonia albiflora Pall., Fl. Ross. 1(2): pl. 84, 1788.

Paeonia edulis Hook., Parad. Lond. pl. 78, (1805) =[1807].

P. edulis Reevesiana Paxt., Mag. Bot. 1: 197, 1834.

$P$. Reevesiana Paxt. in Loud., Hort. Brit., ed. nov. suppl. 601, 1850.

See Lynch, Roy. Hort. Soc., Jour. 12: 428-445, 1890.

Huth, Engler Bot. Jahrb. 14: 258-276, 1891.

Finet \& Gagnepain, Soc. Bot. France, Bul. 51: 523527, 1904.

Komarov, Fl. C.C.C.R. 7: 25-26, 1937.

Stebbins, Univ. Calif., Publ. Bot. 19: 252, 1939.

714. Adelia P. Browne, Civ. Nat. Hist. Jamaica 361, pl. 36, fig. 3, 1756.

Adelia L., Syst. Nat., ed. 10, 2: 1,298, 1759.

Borya Labill., Nov. Holl. Pl. Spec. 1: 81, pl. 107, 1804.

Borya Willd., Sp. Pl. of Linnaeus, ed. 4 by Willd., 4(2): 711, 1806.

Forestiera Poir. in Lam. Encyc. Méth. Bot. Suppl. 1: 132, 1810; 2: 664, 1811.

715. Anelsonia Macbr. \& Pays., Bot. Gaz. 64: 81, 1917.

716. Melobesia membranacea (Esper.) Lamx., Soc. Philom.

Paris, Nouv. Bul. Sci. 3(63): no. 29, 1812.

717. Raillardia rocki Sherff, Bot. Gaz. 95: 79, 1933. Railliardia Rockii Sherff, Bishop Mus., Bul. 135: 130-131, 1935.

Dubautia Rockii (Sherff) Keck, Bishop Mus., Occas. Pap. 11(19): 28, 1936.

See St. John, Pacif. Sci. 4: 342, 1950.

718. Senecio mortuosus Standl. ex Morton, Phytologia 4(4): 262263, 1953.

719. Cystopteris fragilis (L.) Bernh. forma simulans Weatherby, Rhodora 37: 376, 1935. 
720. Didymosphaeria Equiseti-hiemalis Larsen \& Munk, Dansk Bot. Arkiv 14: 17-18, fig. 6, 1952.

721. Cydonia Cydonia (L.) Pers., Syn. Pl. 2: 40, 1807. Pyrus Cydonia L., Sp. Pl. 480, 1753. Cydonia Oblonga Mill., Gard. Dict., ed. 8, Cydonia No. 1, 1768.

C. vulgaris Pers., Syn. P1. 2: 40 corrigenda, 1807.

722. Campanula caulibus diffusis, foliis subovatis integerrimis L., Sp. Pl. 169, 1753.

723. Castalia Leibergi Morong, Bot. Gaz. 13: 124, 1888. Nymphaea Leibergi Morong, Bot. Gaz. 13: 124, 1888.

724. Trymalium ramosissimum Audas, Austral. Bushland 283, 1950.

725. Naegelia Regel, Flora 31: 249, 1848. Nägelia Zoll. \& Mor., Syst. Verz. Zoll. 20, 1845-1846.

726. Eupatorium verticillatum Lam., Encyc. Méth. Bot. 2: 405, 1786.

E. purpureum L., Sp. P1. 838, 1753.

See Farwell, Mich. Acad. Sci., Rept. 20: 192, 1918.

Wiegand, Rhodora 22: 57-70, 1920.

Mackenzie, Rhodora 22: 157-165, 1920; 29: 6-9, 1927.

Wiegand \& Weatherby, Rhodora 39: 297-306, 1937. Jennings, Castanea 7: 43-48, 1942.

Fernald, Rhodora 47: 193, 1945.

727. Solanum vestitum Nutt. ex Seem., Fl. Vit. 174, 1866. S. vestitum Benth. in Maund, Botanist 4: text and pl. 192, 1839.

S. Nelsoni Dunal $\beta$ var. thomasiaefolium Seem., Jour. Bot. Brit. For. 1: 209, 1863.

728. Pandorea J. Agardh in de Toni, Syll. Algar. 6(5): 752, 1924. Pandorea (Endl.) Spach, Hist. Nat. Vég. Phan. 9: 136, 1840.

Pandorea Endl., Gen. Pl. 711, 1836-1840.

729. Anthochloa Nees in Meyen, Reise um Erde 2: 14, 1835. Stapfia Davy, Erythea 6: 109-111, pl. 3, 1898.

Stapfia Chodat, Herb. Boissier, Bul. 5: 939-947, pl. 23, 1897. 
Neostapfia Davy, Erythea 7: 43, 1899.

Davyella Hack., Oesterr. Bot. Zeitschr. 49: 133-134, 1899.

730. Lobelia macrostachys H. \& A., Bot. Beechey Voy. 88, 1832.

Trematocarpus macrostachys (H. \& A.) Zahlbr., K. K.

Naturhist. Hofmus. Wien, Ann. 6(3-4): 430-432, fig.

$1-5,1891$.

Trematocarpus Kuetz., Phyc. Gen. 410, pl. 51, fig. 1, 1843.

Trematolobelia macrostachys (H. \& A.) Zahlbr. in Rock,

Coll. Hawaii Publ., Bul. 2: 45, pl. 11-12, 1913.

Trematolobelia sandwicensis Degener, Fl. Haw. 339:

$10 / 15 /$ '34.

731. Aleurites triloba J. R. \& G. Forst., Char. Gen. Pl. 111-112, pl. 56, 1776.

Camirium [Rumph.] Gaertn., Fruct. 2: 194-196, pl. 125,

1791.

Dryandra Thunb., Fl. Jap. 13, pl. 27, 1784.

Elaeococca A. Juss., Euphorb. Gen. Tent. 38, pl. 11, 1824.

Telopea Soland. ex Baill., Gen. Euphorb. 345, 1858.

732. Debregeasia Gaud., Bot. Voy. La Bonite, pl. 90, (18441866) $=$ [1844].

Leucocnide Miq., Pl. Jungh. 1: 36-37, 1851.

Morocarpus Sieb. \& Zucc., Math. Phys. Acad. Muench., Abhandl. 4(3): 218, 1846.

733. Panicum spinescens R. Br., Prodr. Fl. Nov. Holl. 193, 1810.

Chamaeraphis spinescens (R. Br.) Poir. in Lam., Encyc.

Méth. Bot. Suppl. 2: 189, 1811.

Pseudoraphis spinescens (R. Br.) Vickery, Roy. Soc.

Queensland, Proc. 62(7): 69-71, 1952.

Panicum asperum Koenig, Naturforscher 23: 209, 1788.

Chamaeraphis aspera (Koen.) Nees in Wall., Cat. (Num.

List Pl. E. Ind.), No. 8,679, 1849.

Pseudoraphis aspera (Koen.) Pilger, Bot. Gard. Berlin,

Notizbl. 10: 210, 1928.

Panicum abortivum R. Br., Prodr. Fl. Nov. Holl. 193, 1810. Chamaeraphis abortiva (R. Br.) Poir. in Lam. Encyc. Méth.

Bot. Suppl. 2: 184, 1811. 
Orthopogon abortivus (R. Br.) Spreng., Syst. Veg. of Linnaeus, ed. 16 by Spreng., 1: 306, 1825.

734. Aster Fendleri Gray, Am. Acad. Arts Sci., Mem. II, 4: 66, 1849.

Aster Nuttallii T. \& G. var. Fendleri (Gray) Gray, Pacif.

R. R. Rept. 4(5): 97, 1856.

735. Ixora nigritans R. Br. ex Wall., Cat. (Num. List Pl. E. Ind.)

No. 6,154, 1828.

I. nigritans Wight ex Wall., Cat. (Num. List Pl. E. Ind.)

No. 1,335, 1833.

I. nigritans $\mathrm{R}$. Br. in Wight \& Arn., Prodr. Fl. Penin. Ind.

Orient. 1: 428, 1834.

736. Glochidion J. R. \& G. Forst., Char. Gen. 113-114, pl. 57, 1776.

Bradleia Cav., Icon. Descr. Pl. Hispan. 4: 48, pl. 371, 1797.

Bradleja Banks ex Gaertn., Fruct. 2: 127, pl. 109, 1791. Glochidionopsis Bl., Bijdr. Fl. Nederl. Indië 2: 588, 1825. Glochisandra Wight, Icon. Pl. Ind. Orient. 5(2): 28, pl. 1,905, 1852.

Gynoon A. Juss., Mus. Hist. Nat. Paris, Mém. 10: 335, 1823.

Lobocarpus Wight \& Arn., Prodr. Fl. Penins. Ind. Orient. 7, 1834.

737. Mentha bracteolata Opiz, Seznam 65, 1852.

M. bracteolata Opiz ex Déséglise, Soc. Etud. Sci. Angers, Bul. 1881-1882: 210, 1882.

738. Variegatae Krukoff, Jour. Arn. Arb. 20: 226, 1939.

739. Davia [Boerh.] Mill., Gard. Dict., ed. 8, Pavia, 1768.

See Rickett, Taxon 4: 185-188, 1955.

740. Serrafalcus Lloydianus Gren. \& Godr., Fl. France 3: 591, 1855.

741. Macaranga quadriglandulosa Warb. var. variabilis Perry, Jour. Arn. Arb. 34: 239, 1953.

742. Urostachys sulcinervius (Spring) Hert. var. kanaiensis Hbd. ex Nessel, Die Bärlappgewächse 54, pl. 7, fig. 7, 1939. 
743. Aëtia Adans., Fam. Pl. 2: 84, 1763.

744. Cucurbita Citrullus L., Sp. Pl. 1,010-1,011, 1753.

Citrullus Forsk., Fl. Aegypt. Arab. 167, 1775.

Anguria Mill., Gard. Dict., abr. ed. 4, 1: [83], 1754.

Cococynthis Ludwig, Inst. Regn. Veg., ed. 2, 139, 1757.

Citrullus vulgaris Schrad. in Ecklon \& Zeyher, Enum. Pl.

Afr. Austr. Extratrop. 279, 1836.

See Hara, Bot. Mag. [Tokyo] 61: 716, 1948; Taxon 2:

134-135, 1953;

Fosberg, Taxon 2: 99-101, 1953; 3: 2, 1954; 5: 15, 1956;

Meeuse, Taxon 4: 198, 1955.

745. Quercus mibra L., Sp. PI. 996, 1753.

Q. borealis Michx. f., Hist. Arbres Forest. Am. Sept., Engl.

ed. as N. Am. Sylva 1: 119-121, pl. 26, 1817.

Q. borealis Michx. f. var. maxima (Marsh.) Sarg., Rhodora

18: 48, 1916.

Q. rubra L. var. borealis (Michx. f.) House, New York State

Mus., Bul. 243-244: 60, 1923.

See Sargent, Rhodora 18: 47, 1916.

Mackenzie, Rhodora 30: 235, 1928.

Svenson, Rhodora 41: 522, 1939.

Palmer, Am. Midl. Nat. 27: 732, 1942.

746. Euphorbia maculata L., Sp. PI. 455, 1753.

E. supina Raf., Am. Monthly Mag. Crit. Rev. 2: 119, 1817.

Xamesike supina (Raf.) Raf., Aut. Bot. 97, (1815-1840)=

1840.

See Wheeler, Gray Herb., Contr. 127: 76, 1939; Rhodora 48: 197-200, 1946.

Svenson, Rhodora 47: 273-302, 363-388, 1945.

Croizat, Torrey Bot. Club, Bul. 74: 153-155, 1947.

Fosberg, Torrey Bot. Club, Bul. 74: 332-333, 1947;

Rhodora 55: 241-243, 1953.

747. Thelephora laevis Pers. ex Fries, Syst. Mycol. 1: 451, 1821.

Peniophora laevis (Pers. ex Fries) Burt, Mo. Bot. Gard.,

Ann. 12: 257, (1925) $=$ [1926].

P. affinis Burt, Mo. Bot. Gard., Ann. 12: 266, (1925) =

[1926]. 
See Burt, Mo. Bot. Gard., Ann. 13: 280, $(1925)=[1926]$. Rogers \& Jackson, Farlowia 1: 318, 1943.

748. Mercurialis perennis L., Sp. Pl. 1,035, 1753.

M. montana testiculata Bauh., Pinax 122, 1671.

M. montana spicata Bauh., Pinax 122, 1671.

749. Heterobasidion Bref., Unters. Ges. Myk. 8: 154, (1889) =

[Nov. 1888].

Heterobasidium Massee, Linn. Soc. Lond. Bot., Jour. 25:

127-128, 1889.

750. Musaenda fr frondoso L., Sp. Pl. 177, 1753.

M. frondosa L. ex Stickm., Herb. Amboin. 17, 1754; Linnaeus, Amoen. Acad. 4: 127, 1760.

751. Gynoxis sancti-antonii Cuatr., Fieldiana Bot. 27(1): 9, 1950.

752. Ficus vilamilii (Merr.) Sata, Monogr. Stud. Ficus, Taihoku Imp. Univ., Fac. Agric., Inst. Hort. \& Econ. Bot., Contr. 32(1): 183, 1944.

753. Philacra auriculata Dwyer, Brittonia 5: 126, 1944.

754. Coreopsis tinctoria Nutt., Acad. Nat. Sci. Phila., Jour. 2:

114-115, 1821.

C. tinctoria Nutt. forma atropurpurea (Hook.) Fern., Rhodora 44: 477, 1942.

C. tinctoria Nutt. var. atropurpurea Hook. forma tinctoria

Sherff, Brittonia 6: 341, 1948.

Calliopsis tinctoria (Nutt.) Hook. var. atropurpurea Hook.,

Curtis's Bot. Mag. 63: pl. 3,511, 1836.

755. Cephalanthus occidentalis L. forma lanceolatus Fern., Rhodora 49: 181, 1947.

756. Acinaria Raf., Jour. Phys. Chim. Hist. Nat. 89: 107, 1819;

Isis von Oken 1820: 1: Lit. Anz. 243, 1820. Nitella Ag., Syst. Alg. xxvii, 1824; emend. A. Braun,

Hooker's Jour. Bot. \& Kew Miscell. 1: 193-195, 1849.

See Wood, Torrey Bot. Club, Bul. 75: 283-284, 1948.

757. Peridinium claudicanoides Graham, Carnegie Inst. Wash., Publ. 542: 24, fig. 29, 1942.

758. Labordia Cyrtandrae (Baill.) Skottsb., Hort. Gotoburg, Acta 10: 156, 1936. 
L. Cyrtandrae (Baill.) St. John, Bishop Mus., Occas. Pap. 12(8): 5, 1936.

Geniostoma Cyrtandrae Baill., Soc. Linn. Paris, Bul. 1:

239, 1880.

759. Rhynchospora glauca Vahl var. chinensis (Boeck.) C. B.

Clarke forma spicaeformis (Hbd.) Kükenth. ex Skottsb., Hort. Gotoburg, Acta 2: 213, 1926.

R. glauca Vahl ssp. lavarum (Gaud.) Kükenth. var. spicae-

formis (Hbd.) Kükenth., Engler Bot. Jahrb. 75: 150,

1950.

R. spicaeformis $\mathrm{Hbd}$., Fl. Haw. Is. 477, 1888.

760. Styphelia Banksii (Gaud.) F. Muell., Fragm. Phytogr.

Austral. 6: 57, 1867.

Cyathodes Banksii Gaud., Bot. Voy. Freyc. Uranie 98,

$(1826)=[1827]$.

761. Salix alexensis Cov. ex Briggs, Rhodora 55: 249, 1953.

S. alaxensis (Anderss.) Cov., Wash. Acad. Sci., Proc. 2:

$280,1900$.

S. speciosa H. \& A. $\beta$ Alaxensis Anderss. in A. DC., Prodr. 16(2): $275,1868$.

762. Clermontia montis-Loa Rock, Coll. Hawaii, Bul. 2: 40, pl. 9, 1913.

See Rock, Bishop Mus., Mem. 7(2): 334, 1919.

763. Danthonia spicata pinetorum Piper, Erythea 7: 104, 1899. Meranthrepta pinetorum Piper, U. S. Natl. Herb., Contr. 11:

122, 1906.

M. thermale (Scribn.) Heller ex Fedde \& Schust., Just's Bot.

Jahresber. 37(2): 128, 1911.

D. thermale Scribn., U. S. Dept. Agric., Div. Agrostol., Circ. 30: 5, 1901.

764. Phyllostegia parviflora (Gaud.) Benth., Linnaea 6: 79,

1831.

P. parviflora (Gaud.) Benth. var. Gaudichaudii Gray, Am.

Acad. Arts Sci., Proc. 5: 344, 1861.

Prasium parviflorum Gaud., Bot. Voy. Freyc. Uranie 453,

$(1826)=[1829]$; Atlas pl. 65, fig. 1, 1826.

765. Railliardia scabra DC., Prodr. 6: 441, 1837.

R. scabra DC. var. hispidula Gray, Am. Acad. Arts. Sci.,

Proc. 5: 133, 1861. 
Dubautia scabra (DC.) Keck, Bishop Mus., Occas. Pap. 11(19): 26, 1936.

See Sherff, Brittonia 6: 338, 1948.

St. John, Pacif. Sci. 4: 342, 1950.

766. Betula caerulea-grandis Blanchard, Betula 1(1): one page unnumbered, 1904.

767. Carex chikungana Bailey, Gent. Herb. 1: 13, 1920. Saussurea oxydonta Hultén, Kungl. Svenska

Vetenskapsakad., Handl. III, 8(2): 207, 1930.

768. Cyrtandra velutina Nad., Jour. Bot. (ed. Morot) 13: 4, 1899.

769. Eugenia hawaiiensis Degener, Fl. Haw. 273: 7/15/'32.

770. Lupinus alpicola Henders. in Piper, U. S. Natl. Herb.,

Contr. 11: 355-356, 1906.

771. Nemacladus pinnatifidus Greene, Calif. Acad. Sci., Bul. 1: 197, 1885.

N. ramosissimus Nutt. var. pinnatifidus (Greene) Gray,

Synopt. Fl. N. Am. 2(1): Suppl. 393, 1886.

772. Bidens waianensis Sherff, Bot. Gaz. 70: 104, 1920. B. waianaeensis Sherff emend. Skottsb., Hort. Gotoburg., Acta 15: 508, 1944.

773. Macaranga salomonensis Perry, Jour. Arn. Arb. 34: 210, 1953.

774. Rosa acicularis Lindl. var. lacorum Erlanson, Mich. Acad.

Sci. Arts Letters, Pap. 5(1925): 86, 1926.

R. acicularis Lindl. var. lacuum Erlanson emend. Butters \&

Abbe, Rhodora 55: 169, 1953.

775. Lypochaeto australis Less. var. denticulata Wawra, Flora 56: 77, 1873.

Lipochaeta lobata (Gaud.) DC. var. $y$ denticulata (Wawra)

Sherff, Bot. Gaz. 95: 92, 1933.

See Rickett, Taxon 4: 185-188, 1955.

776. Aporosa Bl., Bijdr. Fl. Nederl. Ind. 12: 514, 1825. Aporusa Bl. emend. Bl. in Bl. \& Fish., Fl. Jav. 1: vi, 1828.

See Croizat, Bot. Gard. Buitenzorg, Bul. III, 17(2): 217, 1942.

777. Odontoglossum auropurpureum Reichb. f., Linnaea 22: 848, 1849. 
O. aureo-purpureum Reichb. f. emend. Reichb. f., Gard.

Chron. n. s. 3: 492, 1875.

See Schweinfurth, Harvard Univ. Bot. Mus., Leafl. 14(3):

63, 1949.

778. Lycopodium complanatum L. Unterart L. chamaecyparissus

A. Braun forma Monstr.-triceps Milde, Acad. Caes.

Leop.-Carol., Nova Acta 26(2): 407, 1858.

See Nessel, Die Bärlappgewächse 329, 1939.

779. Padina Howeana Bфrg., Dansk Bot. Arkiv 2(1): 442, 1920.

Dictyota variegata Lamx., Mus. Hist. Nat. Paris, Ann. 20:

272, 1813; repr. as Essai genres Famille Thalassio-

phytes non articulées 57, 1813.

P. variegata Hauck, Hedwigia 26: 42, 1887.

Zonaria variegata (Lamx.) Martius, Icon. Pl. Cryptog. 6, pl. 2, fig. 2, 1827.

$P$. variegata (Lamx.) Hauck sensu B $\varnothing$ rg., Dansk Bot. Arkiv 1(2): 205, 1914; 2(2): 49, 1914.

See Taxon 3: 234, 1954; 4: 118, 1955.

780. Panicum paludosum Hassk., Pl. Jav. Rar. 16-17, 1848. P. paludosum Roxb., Hort. Beng. 8, 1814; Fl. Ind. 1: 307, 1832.

781. Clerodendron canescens Wall., Cat. (Num. List Pl. E. Ind.) No. 1,804, 1828-1849; Wall. in Schauer in DC., Prodr. 11: $665,1847$.

782. Cyrtandra J. R. \& G. Forst., Char. Gen. 5-6, pl. 3, 1776. Kyrtandra Blanco, Fl. Filip. 18, 1837.

Getonia Banks \& Soland. ex Benn., Pl. Jav. Rar. 122, 1838. Rhynchocarpus Reinw. \& Bl., Cat. Gew. Buitenzorg 84, 1823.

Whitia Bl., Cat. Gew. Buitenzorg 16-17, 1823.

783. Rhynchospora glauca Vahl form 2 condensata Kükenth. in Engler, Bot. Jahrb. 69(2): 259, 1938; Engler Bot. Jahrb. 75: 148, 1950.

784. Perispermon Heydr., West. Ausbau Corall. Syst., Deutsch.

Bot. Ges., Ber. 18: 316, 1900.

Perispermum Degener, Fl. Haw. 307: 8/10/'32. 
785. Styphelia Tameiameiae (Cham.) F. Muell., Fragm. Phytogr. Austral. 6: 57, 1867.

Cyathodes Tameiameiae Cham., Linnaea 1: 539, 1826.

786. Canavalia? galeata Gaud., Bot. Voy. Freyc. Uranie 486, $(1826)=[1830]$.

C. galeata (Gaud.) Gaud. ex Vogel, Linnaea 10: 584, 1836. Dolichos galeatus Gaud., Bot. Voy. Freyc. Uranie 486, $(1826)=[1830] ;$ Atlas pl. 115, 1826.

See St. John, Bishop Mus., Occas. Pap. 15(22): 231-233, 1940.

787. Rhinanthus minor L. ssp. borealis (Sterneck) Löve, Bot. Notis. 1950(1): 52, 1950.

788. Inocarpus J. R. \& G. Forst., Char. Gen. 65-66, pl. 33, 1776. Bocoa Aubl., Hist. Pl. Guiane 2: Suppl, 38, 1775; 4: pl. 391, 1775.

Etballia Benth., Hooker's Jour. Bot. 2: 99, 1840.

- Inodaphnis Miq., Fl. Ind. Bat. Suppl. 357, 1861. Inocarpus fagiferus (Parkins.) Fosb., Wash. Acad. Sci., Jour. 31: 95-96, 1941.

789. Epipremnum humile (Schott) Hook. f., Fl. Brit. Ind. 6: 549, 1893.

Anadryum humile Schott in Miq., Mus. Bot. Lugd. Bat. 1: $127,1893$.

790. Desmos cochinchinensis Lour., Fl. Cochinchin. 352, 1790. D. chinensis Lour., Fl. Cochinchin. 352, 1790.

See Furtado, Gardens' Bull. 9: 226, 1937. Merrill, Am. Phil. Soc., Trans. 24(2): 160-161, 1935.

791. Cyrtandra Clarkei Vatke forma ovatifolia Skottsb., Hort. Gotoburg., Acta 15: 444, 1944.

792. Oenothera argillicola Mackenzie, Torreya 4: 56-57, 1904. Onagra argillicola Mackenzie, Torreya 4: 57, 1904.

793. Persoonia coriacea Audas, Australian Bushland 131, 1950.

794. Ptemula brunneosetosa Corner, Ann. Bot. n. s. 16(64): 535, fig. $1,1952$.

795. Scirpus swampianus Bosc ex Kunth, Enum. Pl. 2: 168, 1837.

796. Delphinium occidentale Wats., Bot. Cal if, 2: 428, 1880. D. elatum L. var. (?) occidentale Wats., Bot. King. Exped. 
D. occidentale (Wats.) Wats., Ewan, Univ. Colo. Stud., ser. D, 2(2): 136-138, 1945.

797. Halymenia Durvillaei Bory var. formosa Weber van Bosse,

Siboga Exped., Monogr. 59b: 235, 1921.

H. formosa Kütz., Tab. Phyc. 16: 33, pl. 91, g, h, 1866.

798. Salvia afr. lutea L., Sp. Pl. 26, 1753.

S. aurea L., Sp. Pl., ed. 2, 1: 38, 1762.

799. Silphium terebinthinaceum Jacq. var. Lucy-Brauniae

Steyerm., Rhodora 53: 134, 1951.

800. Rhus Filicina Moç. \& Sessé ex DC., Prodr. 2: 67, 1825. R. potentillaefolia Turcz., Soc. Nat. Mosc., Bul. 31: 469, 1858.

R. Tetlatziam Sessé \& Moç., Pl. Nov. Esp. 47, 1887; ed. 2, 44, xi, 1893.

Toxicodendron potentillifolium Ktze., Rev. Gen. Pl. 1:

154, 1891.

Actinocheita filicina (DC.) Barkley, Mo. Bot. Gard., Ann.

24: 2-5, 1937.

See Barkley \& Reed, Am. Midl. Nat. 21: 368-377, 1939.

Bullock, Kew Bul. 337-339, 1939.

Furtado, Gardens' Bul. 12: 367-368, 1949.

801. Pinus monophyllus Torr. \& Frém. in Frém., Rept. Expl.

Exped. Rocky Mts. 319, pl. 4, 1845.

P. cembrodes Zucc. var. monophylla (Torr. \& Frém.) Voss,

Deut. Gardenrat Beilage 123, 1904; Deut. Dendrol.

Gesell., Mitt. 16: 95, 1907.

Caryopitys monophylla (Torr. \& Frém.) Rydb., Torrey Bot.

Club, Bul. 32: 597, 1905.

802. Robinia PseudoAcacia L., Sp. Pl. 722, 1753.

R. Pseud-Acacia L. emend. L., Sp. Pl., ed. 2: 1,043-1,044, 1763.

803. Fimbristylis cymosa R. Br. var. pycnocephala Kükenth. in

Skottsb., Göteborg Bot. Trädgård, Meddel. 2: 212,

1926.

F. pycnocephala Hbd., Fl. Haw. Is. 473-474, 1888.

804. Inga urabensis L. Uribe, Caldasia 4: 406, 1947.

805. Saxifraga occidentalis Wats. var. wallowensis Peck, Leafl. W. Bot. 5: 60, 1947. 
806. Quercus virginiana Mill. var. eximea Sarg., Bot. Gaz. 65: 447, 1918.

Q. virginiana Mill. var. eximia Sarg. emend. Dayton in Little, Check List Native Nat. Trees of U. S. 247, 1944; Rhodora 54: 77, 1952.

807. Triticum L., Gen. Pl., ed. 5, 37, 1754.

Tritica L., Sp. Pl. 85, 1753.

808. Glossopetalum Schreb., Gen. Pl. of Linnaeus, ed. 8 by

Schreb., 1: 205, 1789.

Glossopetalon Gray, Pl. Wright. 2: 29, pl. 123, 1853.

Forsellesia Greene, Erythea 1: 206, 1893.

See Ensign, Am. Midl. Nat. 27: 501-511, 1942.

St. John, Biol. Soc. Wash., Proc. 55: 109-112, 1942.

809. Cloëzia Brongn. \& Gris., Soc. Bot. France, Bul. 10: 576577, 1863.

810. Calceolaria Storkii Standl., Field. Mus. Nat. Hist., Bot.

Ser., Publ. 18(3): 1,103, 1938.

811. Canthium barbatum (Forst. f.) Seem. var. australense Fosb. forma tubuai Fosb., Bishop Mus., Occas. Pap. 13(19): 259, 1937.

812. Aconitum variegatum L. B typicum Regel, Ann. Sci. Nat. Bot. IV, 16: 147, 1862.

A. variegatum L. $\alpha$ Canarum (Reichb.) Regel in Reichb.,

Ill. Gen. Acon. pl. 34, 1823-27.

813. Amaryllis Bella donna L., Sp. Pl. 293, 1753.

A. Belladonna L. emend. L., Sp. Pl., ed. 2, 1: 421, 1763.

See Traub \& Moldenke, Amaryllidaceae, 1949.

Dyer, Taxon 3: 72-74, 1954.

Dandy \& Fosberg, Taxon 3: 231-232, 1954.

814. Arnica Lessingii (T. \& G.) Greene, Pittonia 4: 167, 1900. A. angustifolia H. \& A. $\beta$ Lessingii T. \& G., Fl. N. Am. 2: 449, 1843.

A. alpina (L.) Olin \& Ladau var. Lessingii Gray, Syn. Fl. N. Am. 1(2): 383, 1884.

A. Porsildiorum Boivin, Nat. Canad. 75: 210, 1949.

See Rydberg, N. Am. Fl. 34: 328, 1927.

Maguire, Rhodora 52: 281-283, 1950.

Boivin, Rhodora 55: 57, 1953. 
815. Cyperus odoratus L., Sp. Pl. 46, 1753.

C. Ferax L. C. Rich., Soc. Hist. Nat. Paris, Act. 1: 106, 1792.

See Clarke, Linn. Soc. Lond., Jour. 30: 306-307, 1895. Kükenthal, in Engler's Pflanzenreich IV, 20(4): 624, 1936.

816. Capparis cynophallophora L., Sp. Pl. 504, 1753.

C. Jamaicensis Jacq., Enum. PI. Carib. 23, 1760.

C. cynophallophora L., Syst. Nat., ed. 10, 2: 1,071, 1759.

C. flexuosa L., Sp. Pl., ed. 2, 722, 1763.

See Small, Man. S. E. Fl. 577, 1933.

817. Polypodium vulgare L. monstr. acuminatum Copel., Bot.

Gaz. 34: 143-144, 1902.

P. vulgare acuminatum Copel., Bot. Gaz. 34: 143, fig. 4-5, 1902.

818. Goniophlebium Presl, Tent. Pterid. 185, pl. 7, fig. 13-14, 1836.

See Copeland, Gen. Fil. 181, 1947.

Furtado, Lloydia 12: 72, 1949.

819. Cyphokentia samoensis Warb. in Reinecke, Engler's Bot. Jahrb. 25: 588, 1898.

See Furtado, Gardens' Bul. 9: 291, 1937.

820. Smilax Pseudo-China L., Sp. Pl. 1,031, 1753.

S. tamnoides L., Sp. Pl. 1,030, 1753.

S. tamnifolia Michx., Fl. Bor.-Am. 2: 238, 1803.

See Fernald, Rhodora 46: 32-35, 1944.

821. Anapausia Presl, Epim. Bot. 185, 1849.

See Copeland, Gen. Fil. 132, 1947.

822. Boerhavia diffusa L., Sp. PI. 3, 1753.

See Heimerl, Bishop Mus., Occas. Pap. 13(4): 28, 1937.

823. Apera Adans., Fam. P1. 2: 495, 1763. Agrostis L., Sp. Pl. 61-63, 1753; Gen. Pl., ed. 5, 30, 1754.

824. Rhynchosia difformis DC., Prodr. 2: 384, 1825. Arcyphyllum Difforme Ell., Acad. Nat. Sci. Phila., Jour. 1:

371-372, 1818.

See Gleason, Phytologia 2: 212, 1947. 
825. Asplenium nidus L., Sp. Pl. 1,079, 1753. Ophrys Nidus avis L., Sp. Pl. 945, 1753.

826. Astragalus Punjabicus Śirajaev, Brittonia 7: 275-276, 1951. Onobrychis ralphii Śirajaev, Brittonia 7: 275, 1951. Astragalus Albus Sirajaev, Brittonia 7: 276, 1951. A. hircina Jacq. var. indica Śirajaev, Brittonia 7: 276-277, 1951.

827. Asystasia Bl., Bijdr. Fl. Nederl. Ind. 796, $(1825)=[1826]$. 828. Barbarea barbarea (L.) MacMillan, Minn. Geol. \& Nat. Hist. Surv., Bot. Ser., Rept. 1: 259, 1892. Erysimum Barbarea L., Sp. PI. 660, 1753. Barbarea vulgaris R. Br. in Ait., Hort. Kew., ed. 2, 4: 109, 1812.

829. Beautempsia Gaud., Bot. Voy. La Bonite pl. 56, (1844$1866)=[1842]$

830. Bernardia Bernardia (L.) Millsp., Field Columb. Mus., Bot.

2: $58,1900$.

Bernardia carpinifolia Griseb., Fl. Brit. W. I. 45, 1864. B. dichotoma Muell. Arg. $\alpha$ genuina Muell. Arg., Linnaea 34: 172, 1865-1866. Adelia Bernardia (P. Browne) L., Syst. Nat., ed. 10, 1,298, 1759.

831. Brunfelsia mire Monachino, Phytologia 4: 342-347, 1953. 832. Clematis pseudoscabiosaefolia Perrier de la Bathie forma normalis Perrier de la Bathie, Mus. Natl. Hist. Nat. Paris, Not. Syst. 14(4): 309, 1952.

833. Trematosphaeria Morthieri Fuckel, Nassau Ver. Naturk., Jahrb. 25-26: 306, 1871; Symb. Myc. Nacht. 1: 306, 1871.

Zignoella Morthieri (Fuckel) Sacc., Michelia 1: 347, 1878;

Syll. Fung. 2: 222, 1883.

Clypeothecium Weirii Petrak, Ann. Myc. 20: 182, 1922. Trematostoma Morthieri (Fuckel) Shear, Mycologia 270,

1942.

834. Crescentia L., Gen. Pl., ed. 5, 274, 1754.

835. Cucurbita L., Gen. Pl., ed. 5, 441, 1754; Sp. Pl. 1,010$1,011,1753$. 
Cucurbita L., Mill., Gard. Dict., ed. 8, Cucurbita, 1768. Lagenaria Ser., Soc. Phys. Genève, Mem. 3(1): 25-26, pl.

2, 1825; Ser. in DC., Mem. 3: 417, 1881.

Pepo [Tourn.] Mill., Gard. Dict., abr. ed. 4, Pepo, 1754.

See Britton \& Brown, Ill. Fl. N. U. S., ed. 2, 3: 291, 1913.

Bailey, Gent. Herb. 2(2): 78-81, 1929; 2(7): 427-430, 1932.

836. Polemonium occidentale Greene, Pittonia 2: 75, 1890.

P. caeruleum L. ssp. occidentale (Greene) J. F. Davidson,

Univ. Calif., Publ. Bot. 23(5): 225, 1950.

See Brand in Engler, Pflanzenreich IV, 250: 33, 1907.

837. Hernandia labyrinthica Tuyama, Sigenkagaku Kenkyusyo, Bul. 1(1): 42-44, 2 pl., 1943.

838. Trichomanes L., Gen. Pl., ed. 5, 485, 1754; Sp. Pl. 1,097$1,099,1753$.

V andenboschia Copel., Philip. Jour. Sci. 67: 51, 1937.

See Holttum, Gardens' Bul. 12: 304-305, 1937.

839. Polygala verticillata L., Sp. Pl. 706, 1753.

See Fernald, Rhodora 40: 334-338, 1938.

Pennell, Rhodora 41: 378-384, 1939.

840. Cyclomycetella pavonia (Hook.) Murrill, Torrey Bot. Club, Bul. 31: 423, 1904.

Polyporus iodinus Mont., Ann. Sci. Nat. Bot. II, 16: 108, 1841.

P. striatus Mont., Ann. Sci. Nat. Bot. II, 13: 205, 1840. Cycloporellus iodinus (Mont.) Murrill, Torrey Bot. Club,

Bul. 34: 468, 1907; N. Am. Fl. 9(2): 85, 1908.

See Donk, Reinwardtia 1: 485, 1952.

841. Cynodon Dactylon (L.) Pers., Syn. Pl. 1: 85, 1805.

Panicum dactylon L., Sp. Pl. 58, 1753.

Dactylon R. \& S., Syst. Veg. of Linnaeus, ed. 9 by R. \& S., 2: 411, 1817.

Dactilon Vill., Hist. Pl. Dauph. 2: 69, 1787.

842. Cyperus Papyrus L., Sp. Pl. 47, 1753.

843. Cyrtandra olona C. N. Forbes, Bishop Mus., Occas. Pap. 8(3): 34, pl. 5, 1920. 
844. Destrugesia Gaud., Bot. Voy. La Bonite pl. 57, (1844$1866)=[1842]$.

Destruguezia Gaud. ex B. \& H., Gen. Pl. 1: 109, 1862.

845. Didymella Ilicis Larsen, Dansk Bot. Arkiv 14(7): 21-22, 1952.

846. Eleocharis capitata R. Br., Prodr. Fl. Nov. Holl. 1: 225, 1810.

Scirpus capitatus L., Sp. Pl. 48, 1753.

S. capitatus Willd., Sp. Pl. of Linnaeus, ed. 4 by Willd., 1: 294, 1797.

See Clarke, Linn. Soc. Lond. Bot., Jour. 30: 310, 1894.

Blake, Rhodora 20: 24, 1918.

Farwell, Rhodora 32: 180-181, 1930.

Furtado, Gardens' Bul. 9: 293-294, 1937.

847. Solidago ulmifolia Muhl. var. Palmeri Cronq. in Gleason,

New Britton \& Brown Ill. Fl. N. E. U. S. \& Can. 3:

428, 1952.

S. ulmifolia Muhl. var. palmeri Cronq., Rhodora 57: 36,

1955.

848. Schmidtia Moench, Meth. Pl. Hort. Marburg., Suppl. 217,

1802.

Schmidtia Tratt., Fl. Austr. 1: 12-13, pl. 451, 1816.

Schmidtia Steud. in J. A. Schmidt, Beitr. Fl. Cap. Verd.

Ins. 144-146, 1852.

Schmidia Wight, Icon. Pl. Ind. Orient. 16, pl. 1,848, 1852.

849. Halymenia Durvillaei Bory var. edentata Weber-van Bosse,

Siboga Exped., Monogr. 59b: 236, 1921.

850. Hibiscus caerulescens Baill. var. Humbertiellus Hochr.,

Candollea 12: 176, 1949.

Perrierophytum Humbertianthus Hochr., Candollea 12: 181,

1949.

Humbertiella Pseudohenrici Hochr., Candollea 12: 185,

1949.

851. Hibiscus mandrarensis Humbert in schedulis, Hochr., spec. nov. (e § Lilibiscus), Candollea 12: 175, 1949.

852. Lagenifera Cass., Soc. Philom. Paris, Bul. Sci. 199, 1816. Lagenofera Cass., Soc. Philom. Paris, Bul. Sci. 34, 1818.

See Davis, Linn. Soc. N. S. W., Proc. 75: 122, 1950. 
853. Leiacina Raf., Neogenyton 4, 1825.

Tolypella A. Braun, Fragm. Monogr. Charac., ed. Norstedt, 93, 1882.

See Wood, Torrey Bot. Club, Bul. 75: 283, 1948.

854. Matricaria matricarioides (Less.) Porter, Torrey Bot. Club,

Mem. 5: 341, 1894.

Artemisia matricarioides Less., Linnaea 6: 210, 1831.

Matricarioides Less., Linnaea 6: 210, 1831.

Matricarioides Spach, Hist. Vég. Phan. 10: 24, 1841.

855. Meliola gregoriana Stevens, Bishop Mus., Bul. 19: 39, 1925. M. forbesii Stevens, Bishop Mus., Bul. 19: 29, 1925.

856. Pachypodium Nutt. ex T. \& G., Fl. N. Am. 1: 96, 1838.

857. Phaius Lour., Fl. Cochinch. 529, 1790.

Phajus Hassk., Cat. Pl. Hort. Bog., ed. alt., 41-42, 1844.

See Rickett, Taxon 4: 185-188, 1955.

858. Polypodium Dryopteris L. $\beta$ P. Robertianum Hoffm. ex

Hook. \& Bak., Syn. Fil. 309, 1874.

$P$. Robertianum Hoffm., Deutschl. Fl. 2: unnumbered 231st. page, 1796.

859. Pothuava Gaud., Bot. Voy. La Bonite pl. 116-117, (18441866) $=[1851]$.

860. Silene antirrhina L., Sp. Pl. 419, 1753.

S. Antirrhina L., C. L. Hitchc. \& Maguire, Univ. Wash.,

Publ. Biol. 13: 14, 1947.

861. Tacca maculata Zipp. ex Span., Linnaea 15: 480, 1841. T. maculata Seem., Fl. Vit. 103, 1865.

862. Vilfoidea (Rouy) Beetle, Univ. Wyo., Publ. 15(2): 32, 1950. Vilfoidea Rouy, Fl. France 14: 59, 1913.

863. Columellia Ruiz \& Pavon, Fl. Peruv. Prodr. 3, pl. 1, 1794.

864. Sarmienta Ruiz \& Pavon, Fl. Peruv. Prodr. 4, 1794.

865. Sanchezia Ruiz \& Pavon, Fl. Peruv. Prodr. 5, pl. 32, 1794.

866. Schizanthus Ruiz \& Pavon, Fl. Peruv. Prodr. 6, 1794.

867. Senecio Guadalupe Cuatr., Fieldiana Bot. 27(2): 23, 1951.

868. Ulmus mubra Muhl., Am. Phil. Soc., Trans. 3: 165, 1793.

U. fulva Michx., Fl. Bor.-Am. 1: 172, 1803.

See Fernald, Rhodora 47: 203-204, 1945. 
869. Cyrtandra Taitensis Rich ex Gray, Am. Acad. Arts Sci., Proc. 6: 39, 1866.

C. tahitensis Nad., Enum. Pl. Tahiti 60, 1873.

C. Nadeaudi C. B. Clarke, in DC., Monogr. 5(1): 264-265, 1883.

870. Triumfetta L., Gen. Pl., ed. 5, 203, 1754; Sp. Pl. 444, 1753. 871. Euphorbia Celastroides Boiss. var. Odonatoides Sherff, Am. Jour. Bot. 38: 55, fig. 2, 1951.

872. Crateva L., Gen. Pl., ed. 5, 203, 1754; Sp. Pl., ed. 2, 636637, 1762.

Crataeva L., Gen. Pl., ed. 5, index, 1754; ed. 6, 238, 1764; Syst. Nat., ed. 13, 2: 326, 1770.

Crataeua L., Syst. veg. of Linnaeus, ed. 13 by Gmelin, index, 1770.

See Rickett, Taxon 4: 185-188, 1955.

873. Bellonia L., Gen. Pl., ed. 5, 79, 1754; Sp. Pl. 172, 1753.

874. Rhynchospora pseudo-Sellowiana Kükenth., Bot. Jahrb. 75:

$155,1950$.

R. Sellowiana Steud., Nom., ed. 2, 2: 456, 1841; Syn. PI.

Cyp. 145, 1855.

R. Selloiana Boeck., Linnaea 37: 620, 1871-1873.

R. filifolia Kunth, Enum. Pl. 2: 299, 1837.

R. filifolia Gray in Torr., Lyc. Nat. Hist. New York, Ann.

3: 366, 1836.

875. Juglans microcarpa Berland. in Berland. \& Chovel, Diario

Viage Comision de Limites bajo Mier y Teran 276, 1850.

J. rupestris Engelm. ex Torr. in Sitgreaves, Rept. Exped.

Zuni \& Colo. Rivers 171, pl. 15, 1853. (32nd. U. S.

Congr. Senate, Exec. No. 59).

J. nana Engelm., Am. Assoc. Adv. Sci., Proc. 5: 226, 1851.

J. Whippleana Bigel. ex Engelm. in Sitgreaves, Rept. Exped.

Exped. Zuni \& Colo. Rivers 171, 1853.

See Johnson, Jour. Arn. Arb. 25: 436, 1944.

876. Monarda punctata L. ssp. villicaulis Pennell, Torrey Bot. Club, Bul. 46: 186, 1919.

M. punctata L. ssp. villicaulis (Pennell) Epling. Madro ño 3: 24, 1935. 
M. punctata L. var. villicaulis Pennell ex Palmer \&

Steyerm., Mo. Bot. Gard., Ann. 22: 634, 1935.

M. punctata L. var. villicaulis (Pennell) Shinners, Field \&

Lab. 21: 90, 1953.

877. Baccharis cundinamarcenssis Cuatr., Mutisia 17: 4, 1953.

878. Bupariti Duhamel, Sem. Pl. Arbres, Addit. 5, 1760.

Thespesia Soland. ex Correa, Mus. Paris, Ann. 9: 290, pl.

8, fig. 2, 1807.

See Rothmaler, Fedde Repert. 53: 6-7, 1944.

Taxon 3: 118, 156, 233, 1954.

879. Aconitum fudjipedis Nakai, Natl. Sci. Mus. (Tokyo), Bul.

32: 28, 1953.

880. Stenoloma Fée, Gen. Fil. or Mém. Fam. Foug. 5: 203, 330, $(1850-1852)=[1852]$.

See Pichi-Sermolli, Webbia 9: 361-362, 1953; Taxon 3: $71,1954$.

881. Ilex Paraguariensis St.-Hil., Mus. Hist. Nat. Paris, Mém. 9: 351, 1822.

I. Mate St.-Hil., Hist. Pl. Remarq. Brésil \& Parag. 1: xli, 1824.

I. Curitivensis Miers, Ann. Mag. Nat. Hist. III, 8: 393-394, 1861; repr. as Hist. Maté Pl. 14-15, 1861.

I. Paraguariensis St.-Hil. var. $\alpha$ genuina Loes., Monogr.

Aquifol. 1: 304-306, 1901.

See Parold \& Grondona, Revista Argent. Agron. 16: 199204, fig. 1-2, 1949.

882. Astragalus mollissimus earlei (Greene) Tidestrom, Biol. Soc. Wash., Proc. 48: 40, 1935.

A. Earlei Greene in Rydb., N. Am. Fl. 24(7): 444, 1929. A. Humboldtii sensu M. E. Jones, Rev. Astragal. 232, 1923, non Gray (1864).

883. Althaea foliis simplicibus acuminatis acute dentatis tomentosis Mill., Gard. Dict., ed. 8, Althaea No. 1, 1768.

884. Veronica catenata Pennell, Rhodora 23: 37, 1921.

V. connata glaberrima Pennell, Scroph، E. Temp. N. Am. 368-370, 1935. 
V. comosa Richter var. glaberrima (Pennell) Boivin, Nat. Canad. 79: 174, 1952.

885. Capparis cordifolia Lam., Encyc. Méth. Bot. 1: 609, 1785. C. mariana Jacq., Pl. Rar. Hort. Caes. Schoenbrunn. Descr. Icon. 1: 57, pl. 109, 1797.

Blumea grandiflora Zipp. ex Spanoghe, Linnaea 15: 165, 1841; Miq., Fl. Nederl. Ind. 1(2): 100, 1860.

C. spinosa L. var. Mariana (Jacq.?) K. Schum., Engler Bot. Jahrb. 9: 201, 1888; Schum. \& Lauterb., Fl. Deut. Schutzgeb. 335, 1901.

886. Aponogeton oblongus Troupin, Jard. Bot. État Bruxelles, Bul. 23(3-4): 224-225, 1953.

887. Sorbus americana Marsh., Arbust. Am. 145, 1785. S. americana Pursh, Fl. Am. Sept. 1: 341, 1814. S. micrantha Dum.-Cours., Bot. Cult., ed. 2, 5: 464, 1811. S. microcarpa Pursh, Fl. Am. Sept. 1: 341, 1814. Pyrus microcarpa (Pursh) DC., Prodr. 2: 636-637, 1825. Pymus americana (Pursh) DC., Prodr. 2: 637, 1825.

See Jones, Rhodora 55: 358-360, 1953.

888. Apionema obovatum Nutt., Hillebrand, Fl. Haw. Is. 179, 1888.

Straussia Kaduana (C. \& S.) Gray, Am. Acad. Arts Sci., Proc. 4: 43, 1860.

Coffea Kaduana C. \& S., Linnaea 4: 33, 1829.

889. Berberis canadensis Pursh, Fl. Am. Sept. 219, 1814.

See Fernald, Rhodora 44: 31, 1942.

890. Potentilla pensylvanica L., Mant. 1: 76, 1767.

See Fernald, Rhodora 44: 31, 1942.

891. Drosera longifolia L., Sp. PI. 282, 1753.

D. anglica Huds., Fl. Angl., ed. 2, 135, 1778.

D. intermedia Hayne, in Schrader's Neues Jour. 3(1): 37-39, 1800.

See Diels, in Engler, Pflanzenreich IV, fam. 112: 83-84, 96-97, 1906.

892. Eupatorium Benjamin-Lincolnii Steyerm., Fieldiana Bot. 28(3): 635, 1953. 
893. Euphorbia (bezw. Chamaesyce [nutans var.?] pseudo-nutans

Thell. in Aschers. \& Graebn., Syn. Mitteleurop. Fl. 7(92): 431, 1917.

894. Blumea (an Pluchea ?) somaliensis Thell., Zürich Naturf. Gesell., Vierteljahrsschr. 68: 443-444, 1923.

895. Sida paniculata L., Syst. Nat., ed. 10, 2: 1,145, 1760. S. panniculata L. ex. K. Schum. in Mart., Fl. Brasil. 12(3): 293, 1891.

896. Urera Bequaertii de Wild. in herb. ex Hauman, in Fl. Congo Belge \& Ruanda-Urundi 1: 184, 1948.

897. Sassafras Tzumu (Hemsley) Hemsley, Kew Bul. 55, 1907. Lindera T zumu Hemsl., Linn. Soc. Lond. Bot., Jour. 26: 392, 1891.

Litsea laxiflora Hemsl., Linn. Soc. Lond., Bot., Jour. 26: 383, pl. 8, 1891.

Lindera laxiflora Hemsl. ex Lecomte, Mus. Paris, Nouv. Archiv V, 5: 117, 1913.

Pseudosassafras Tzumu (Hemsl.) Lecomte, Not. Syst. 2: 269, 1911.

P. laxiflora (Hemsl.) Nakai, Jour. Jap. Bot. 16: 126, 1940. See Keng, Taiwan Mus., Quart. Jour. 6: 78-85, 2 pl. 1953.

898. Gouldia terminalis (H. \& A.) Hbd. var. antiqua Fosb. forma kauensis Fosb., Bishop Mus., Bul. 147: 55, 1937.

899. Carex mund Boott var. mundaeformis Koyama, Acta Phytotax. Geobot. 15(4): 113, 1954.

900. Gouania Hillebrandi Oliver in Hbd., Fl. Haw. Is. 83, 1888. Pleuranthodes Hillebrandti (Oliver) Weberb. in Engler \& Prantl, Nat. Pflanzenfam. IV, 3(5): 424, 1896. $P$. hillebrandii (Oliver) Weberb. ex Suessenguth, Engler \& Prantl, Nat. Pflanzenfam., ed. 2, 20d: 166, 1953. Lupulus orbicularis Ktze., $\beta$ Hillebrandtii (Oliver) Ktze., Rev. Gen. Pl. 1: 119, 1891.

901. Dalea enneandra Nutt., in Fraser's Cat. 1813; repr., Pittonia 2: 117, 1890.

D. laxiflora Pursh, Fl. Am. Sept. 2: 741, 1814.

See Shinners, Rhodora 57: 290-293, 1955;

Cronquist et al., Rhodora 58: 23-24, 1956; 
Cronquist, Rhodora 59: 100, 1957;

Shinners, Rhodora 58: 281-289, 1956;

Graustein, Rhodora 58: 20-22, 1956.

902. Rhopalanthus Lindb., Notis. Sällskap. Pro Fauna \& Fl.

Fennica, Förhandl. 13: 390-391, 1874.

Rhossalanthus mnioides Lindb. in Austin, Torrey Bot.

Club, Bul. 6: 21, 1875.

Rhopalanthus Lindb. in Austin, Torrey Bot. Club, Bul. 6: $304,1879$.

903. Pteris ternifolia Cav., Descr. Pl. 266, 1802.

Pellaea ternifolia (Cav.) Link, Fil. Sp. Hort. Bot. Berol.

59, 1841.

904. Sida Meyeniana Walp., Rel. Meyen., Acad. Caes. Leop.-

Carol. Nat. Curios., Nov. Act. 16: suppl 2: 307, 1842.

S. Meyeniana Walp. $\alpha$ var. genuina Hochr., Conserv. \& Jard.

Bot. Genève, Ann. 6: 39, 1902.

Anoda ovata Meyen, Reise Erde Prinzess Louise 2: 139,

1835.

905. Sida lepida F. Muell., Fragm. Phytograph. Austral. 6: 168,

1868.

S. lepidota Gray, Pl. Wright. 18, 1852.

906. Crescentia Cujete L., Sp. P1. 626, 1753.

Cuiete Adans., Fam. P1. 2: 207, 1763.

907. Bergia capensis L., Mant. 2: 241, 1771.

908. Suaeda Forsk., F1. Aegypt.-Arab. 69-71, 1775; Niebuhr,

Icon. Rerum Natur. pl. 18B, 1776.

Dondia Adans., Fam. Pl. 2: 261, 1763.

Lerchea Hall., Hort. Goett. 21, 1743; Rueling, Ord. Pl. 45,

1774.

Cochliospermum Lag., Mem. Pl. Barill. 55, 1817.

Belowia Moq. in DC., Prodr. 13 (2): 168, 1849.

Suaeda vermiculata Forsk., F1. Aegypt.-Arab. 70, 1775;

Forsk. ex J. F. Gmel., Syst. Nat. of Linnaeus, ed. 13

by J. F. Gmel., 2: 503, 1791.

See Uster, Delect. Opusc. Bot. 2: 460, 1793.

Brenan, Taxon 3: 65, 1954.

Burtt \& Lewis, Kew Bul. 387, 1954. 
909. Microoecia Hook. f., Linn. Soc. Lond., Proc. 1: 278, 1849.

910. Passiflora mariquitensis Mutis ex Uribe, Mutisia 21: 1-5, pl. 1, 1954.

911. Lithospermum incanum Forst. f., Fl. Ins. Austral. Prodr. $12,1786$.

Heliotropium anomalum H. \& A., Bot. Beechey Voy. 66, 1832.

H. incanum (Forst. f.) Skottsb., Göteborg. Bot. Trädgärd, Meddel. 2: 259, 1926.

912. Panicum affine H. \& A., Bot. Beechey Voy. 100, 1841. $P$. affine Poir. in Lam., Encyc. Méth. Bot. Suppl. 4: 273, 1816.

P. lanaiense Hitchc., Bishop Mus., Mem. 8(3): 189, 1922.

$P$. Colliei Endl., Wien. Mus. Naturgesch., Ann. 1: 157, 1836.

913. Daedalacanthus nervosus (Vahl) T. Anders., Linn. Soc. Lond., Jour. 9: 487, 1867.

Justicia nervosa Vahl, Enum. Pl. 1: 164, 1804.

Eranthemum nervosum (Vahl) R. Br. ex R. \& S., Syst. Veg.

of Linnaeus, ed. nov. by R. \& S., 1: 174, 1817.

E. pulchellum Andrews, Bot. Reposit. 2: pl. 88, [1798].

See Fosberg, Taxon 2(6): 135-136, 1953.

914. Citharexylum spinosum L., Sp. P1. 625, 1753.

See Britton \& Wilson, New York Acad. Sci., Sci. Surv. Porto Rico 6: 146, 1925.

915. Duckera Barkley, Am. Midl. Nat. 28: 472, 1942.

Rhus, sect. Melanocarpae Engler, Bot. Jahrb. 1: 380, 1881.

916. Premna integrifolia L., Mant. 2: 252, 1771.

P. serratifolia L., Mant. 2: 253, 1771.

Cornutia corymbosa Burm. f., Fl. Ind. 132, 1768.

P. corymbosa (Burm. f.) Rottl. \& Willd. ex Merr., Int.

Rumph. Herb. Amb. 450-451, 1917.

P. corymbosa Rottler, Ges. Naturf. Fr. Berlin, Neue Schr.

4: 187, 1803.

P. obtusifolia R. Br., Prodr. Fl. Nov. Holl. 512, 1810.

See Fosberg, Taxon 2(4): 88-89, 1953. 
917. Docanthe alba O. F. Cook, Nat. Hort. Mag. (Am.) 22: 96, 150, 1943.

918. Ipomaeella A. Cheval., Rev. Bot. Appliq. 30: 272, 1950.

919. Sechium P. Browne, Civ. Nat. Hist. Jamaica 355, 1756.

Chocho Adans., Fam. P1. 2: 500, 1763.

Chayota Jacq., Select. Am. ed. Pict. 124, pl. 245, 1780.

920. Maerua oblongifolia (Forsk.) Rich. var. pallida Pirota, Bruxelles Jard. Bot. État, Bul. 24(2): 138, 1954.

921. Areca cathecu L., Sp. P1. 1,189, 1753. A. Catechu Stickm., Herb. Amb. 6, 1754. Minosa Catechu L. f., Suppl. 439, 1781.

922. Eichornia Kunth, Enum. P1. 4: 129-132, 1843.

Piaropus Raf., Fl. Tell. 2: 81, 1836.

923. Lepidococcus Wendl. \& Druce in Kerchove de Denterghem, Les Palmiers, 249, in indice, 1878 .

See Drude in Mart., Fl. Bras. 3 (2): 292, 1881. Hawkes, Bot. Est. Sao Paulo, Brasil, Arq. 2: 1-2, 1952.

Dugand, Mutisia 20: 1-2, 1954.

924. Acer Wilsoni Rehder in Sarg., Trees \& Shrubs 157, pl. 79, 1902.

A. Wilsonii Rehd. emend. Lawrence, Baileya 2: 88, 1954.

925. Peltophorum pterocarpum (DC.) Backer ex K. Heyne, Nutt.

Pl. Ned. Ind., ed. 2, 2: 755, 1927.

Inga pterocarpa DC., Prodr. 2: 441, 1825.

Caesalpinia inermis Roxb., Hort. Beng. 90, 1814.

Poinciana Roxburghii G. Don, Gen. Syst. 2: 433, 1832.

C. ferruginea Dcne., Mus. Paris, Nouv. Ann. 3: 462, 1834.

Peltophorum ferrugineum (Dcne.) Benth., Fl. Austral. 2:

279, 1864.

P. inerme (Roxb.) Naves in Blanco, Fl. Filip., ed. 3, pl.

335, 1877-1883.

Baryxylum inerme (Roxb.) Pierre, Fl. Forest. Cochinch. pl. $390,1899$.

Peltophorum Roxburghii(G. Don) Degener, F1. Haw. 169b: 12/21/' 38 .

See Blake, Austral. Jour. Bot. 2: 112, 1954. 
926. Echites laxa Ruiz \& Pavon, Fl. Peruv. 2: 19, pl. 134, 1799. Mandevilla suaveolens Lindl., Edwards Bot. Reg. 26: pl.

7, 1840.

M. laxa (R. \& P.) Woodson, Mo. Bot. Gard., Ann. 20: 695696, 1933.

Echites suaveolens (Lindl.) A. DC., Prodr. 8: 452, 1844. Amblyanthera suaveolens (Lindl.) Muell. Arg., Linnaea 30: $447,1860$.

M. Tweedieana Gadeceau \& Stapf, Soc. Sci. Ouest Fr., Bul. III, 3: 2-3, 1913.

927. Potentilla fruticosa L., Sp. Pl. 495, 1753.

Pentaphylloides fruticosa (L.) Schwarz, Thüring. Bot. Ges., Mitt. 1: 105, 1949.

Potentilla arbuscula D. Don, Prodr. Fl. Nepal. 256, 229$230,1825$.

Pentaphylloides arbuscula (D. Don) Löve, Sv. Bot. Tidskr. 48: 223, 1954.

Dasiphora riparia Raf., Autikon Bot. 167, 1838. Potentilla Loureironis Tratt., Rosacearum Monogr. 4, 1824. Potentilla floribunda Pursh, Fl. Am. Sept. 355, 1814. Potentilla fruticosa L. forma villosissima Fern., Rhodora

37: 292, 1935.

Pentaphylloides floribunda (Pursh) Löve, Sv. Bot. Tidskr. 48: 224, 1954.

928. Alternanthera Forsk., Fl. Aegypt.-Arab. 28, 1775. A. achyranth. Forsk., Fl. Aegypt.-Arab. 28, 1775. A. Achyrantha R. Br., Prodr. 1: 417 (or 273), 1810.

929. Fissenia mentzeloides R. Br. ex Harv., Thes. Cap. 1: 61, 1859.

Kissenia spathulata R. Br. ex T. Anders., Linn. Soc. Lond., Jour. 5: Suppl. 1: 43, 1860.

F. Capensis R. Br. ex Endl., Gen. Pl. Suppl. 2: 76, 1842;

R. Br. ex Walp., Rep. 2: 228, 1843.

Cnidone Mentzeloides E. Mey. ex Presl, K. Böhm. Ges. Wissensch., Abhandl. V, 3: 73, 1845; repr. as Bot. Bemerk. 73, 1845.

930. Lactuca massaviensis (Fresen.) Sch. Bip. ex A. Rich., Tent. Fl. Abyss. 1: 460, 1847. 
Brachyramphus lactucoides T. Anders., Linn. Soc. Lond. Bot. 5: Suppl. 23, 1860.

Heterachaena massauensis Fresen., Mus. Senckenb. 3: 74, 1839.

931. Scutellaria elliptica Muhl., Am. Phil. Soc., Trans. 3: 173, 1793.

S. elliptica Mühl. ex Biehler, Pl. Nov. Herb. Spreng. Cent. 26, 1807.

S. elliptica Mühl. ex Spreng., Mant. Prim. Fl. Hal. 44, 1807. S. integrifolia L., Sp. P1. 599, 1753.

S. pilosa Michx., Fl. Bor.-Am. 2: 11, 1803.

S. ovalifolia Pers., Syn. Pl. 2: 136, $(1807)=[1806]$.

S. nemorosa Raf., Am. Monthly Mag. II, 2: 120, 1817.

S. teucrifolia Sm., in Rees Cyclop. 32: Scutellaria No. 15, 1819.

S. pilosa Michx. $\beta$ ovalifolia (Pers.) Benth. in DC., Prodr. 12: 423, 1848.

S. ovalifolia Pers. ssp. mollis Epling, Univ. Calif., Publ. Bot. 20 (1): 86, 1942.

See Fernald, Rhodora 47: 200-203, 1945.

932. Pandanus platyphyllus Martelli, Webbia 2: 439, 1907; 4(1): 27, 1913.

P. platiphyllus Martelli, Webbia 4(2): pl. 28, fig. 34-37, 1914.

933. Echites Echites (L.) Britton in Small, Fl. Miami 147, 200, 1913.

Tabernaemontana Echites L., Syst. Nat., ed. 10, 2: 945. 1759.

934. Ochrosia borbonica J. F. Gmel., Syst. Nat. of Linnaeus, ed.

13 by J. F. Gmel., 2(1): 439, 1791.

Ochrosia Juss., Gen. Pl. 144-145, 1789.

O. maculata Jacq., Collect. 4: 218, 1790; Icon. P1. Rar. 2:

pl. 321, 1792-1793.

Cerbera borbonica (Juss.) Spreng., Syst. Veg. of Linnaeus, ed. 16 by Spreng., 1: 642, 1825.

C. maculata (Jacq.) Willd., Sp. Pl. of Linnaeus, ed. 4 by Willd., 1 (2): 1,223, 1797. 
C. platyspermos Gaertn., Fruct. 2: 193, pl. 124, fig. o-r, 1791.

C. undulata Andrews, Bot. Repos. pl. 130, 1798.

935. Crataegus galbana Beadle, Biltmore Bot. Stud. 1: 74, 1902. C. pexa Beadle, Biltmore Bot. Stud. 1: 116, 1902.

C. senta Beadle, Bot. Gaz. 30: 341-342, 1900 。

See R. M. Harper, Ala. Acad. Sci., Jour. 23-24: 134, 1953.

936. Konig Adans., Fam. Pl。2: 23, 1763.

Koniga R. Br., in Denh. \& Clapp., Narr. Exp. Afr. App. 214, 1826; repr. p. 9.

937. Fe stuca Halleri All., Fl. Ped. 2: 253, 1785.

938. Gillespiea A. C. Sm., Bishop Mus., Bul. 141: 158, 1936.

939. CLERODENDRUM CAPITATUM var. VANDERYSTI

Moldenke, Phytologia 3: 407, 1950.

940. Arisarum Libani Schott, Prodr. Syst. Aroid. 21, 1860.

941. Cyrtandra kalichii tristis (Hbd.) Rock, Am. Jour. Bot. 6: 64-65, 1919.

942. Robinia Caragana L., Sp. Pl. 722, 1753.

943. Fusicocum Corda in Sturm, Deutschl. Fl. 3 abt., 1 (9): 111, 1829.

944. Zschokkea Muell. Arg. in Mart., Fl. Bras. 6(1): 20, pl. 6, 7, 1860.

Zschokkia B. \& H., Gen. Pl. 2: 694, 1876.

945. Grifola Tuckahoe Güss., Mycologia 11: 109, 1919.

946. Hypnum gracile lancastriense Sull. \& Lesq., Musc. Bor. Am. 278, 1856.

947. Braussonetia papyrifera (L.) L'Her. ex Masamune, Kanazawa Univ., Sci. Rept. 2(2): 64, 1954; repr. as Enum. Tracheophyt. Ryukyu Ins. 4: 6, 1954.

Morus papyrifera L., Sp. Pl. 986, 1753.

Broussonetia papyrifera (L.) L'Her. ex Vent., Tabl. Regn.

Veg. 3: 548, 1799.

Broussonetia secundiflora Ortega, Hort. Matr. 61-62, pl. 7,

1798. 
948. Philadelphus oreganus Nutt. ex T. \& G., F1. N. Am. 1: 595, 1840.

See Hu, Jour. Arn. Arb. 36: 85, 1955.

949. Swietenia Mahagoni (L.) Jacq., Enum. Syst. Pl. Carib. 20,

1760; ed. 2, 20, 1762; Index Regn. Veg. 117, 1770. Cedrella Mahag.L., Syst. Nat., ed. 10, 2: 940, 1759.

S. Mahagoni (L.) L., Sp. Pl., ed. 2, 1: 548, 1762.

S. Mahogoni L. ex Lam., Encyc. Méth. Bot. 3: 678-679, $(1789)=[1791]$.

See Little, U. S. Dept. Agric., Handb. 41: 411, 1953.

950. Suaeda australis (R. Br.) Moq. var. nova zelandica J. B.

MacKay \& V. J. Chapman, Roy. Soc. N. Z., Trans.

$82(1): 42-43,1954$.

S. fruticosa Forsk., Fl. Aegypt.-Arab. 70, 1775.

Salsola fruticosa (L.) Forst. f., Fl. Ins. Austral. Prodr. 21, 1786.

Salsola fruticosa L., Mant. 2: 347, 1771.

951. Hutchinsia [R. Br. in] Ait., Hort. Kew., ed. 2, 4: 82, 1812. Hutschinsia D. Dietr., Syn. Pl. 1: 579, 1839.

Hutchina Wight \& Arn. in Wight, Contrib. Bot. Ind. 34, 1834. Hutchinsia Agardh, Syn. Alg. Scand., xxvi, 53, 1817. Hutschinsia R. Br. ex Reichenb. in Mössler, Handb.

Gewächsk. 2(2): 1,124, 1828.

Hutchintia Bory in Duperr., Voy. La Coquille Crypt. 224, 1828.

952. Philadelphus triflorus Wall., Cat. (Num. List Pl. E. Ind.)

No. 3,653, 1831.

P. triflorus Wall. ex Royle, Ill. Bot. Him. 1: 215-216, 1835.

P. triflorus Wall. ex Loudon, Arb. Frut. Brit. 2: 955, 1838.

P. triflorus Wall. ex Koch in Wochenschr. Gärtn. Pfl. 2:

$228,1859$.

P. nepalensis Koehne, Dendrol. 183, 1893.

See C. Schneider, Ill. Hdb. Laubholzk. 1: 373, 1906. Hu, Arn. Arb., Jour. 36: 89-90, 1955.

953. Triticum trachycaulum Link, Hort. Reg. Bot. Descr. 2: 189, 1833.

Elymus trachycaulus (Link) Gould ined., Rhodora 56: 28, 1954. 
T. pauciflorum Schwein. in Keating, Narr. Exped. St.

Peter's River 2: 383, 1824.

Agropyron pauciflorum (Schwein.) Hitchc., Am. Jour. Bot.

21: 132, 1934.

Elymus pauciflorus Lam., Tabl. Encyc. 1: 207, 1791; Poir.

in Lam., Encyc. Méth. Bot. Suppl. 2: 547, (1811)=

[1812].

Agropyrum pauciflorum Schur, Siebenb. Ver. Naturw., Verh. 10: $77,1859$.

954. Tectaria filix mas (L.) Cav., Descr. Pl. 251, 1802.

Polypodium filix mas L., Sp. Pl. 1,090, 1753.

Dryopteris filix mas (L.) Schott, Gen. Fil., unnumbered pl. 9, 1834.

Polystichum Felix Mas (L?) Roth, in Roem. Arch. 2(1):

106, 1799.

Aspidium Filix mas (L.) Sw. in Schrad., Jour. 1800 (2): 38,

1801.

Nephrodium Filix mas (L.) Rich. in Marthe, Cat. Jard. Méd.

Paris 129, 1801.

Lophodium Filix-mas (L.) Newm., Phytol. 4: app. XX, 1851.

955. Pandanus ornatus (Gaud.) Kurz, Roy. Asiat. Soc. Bengal,

Jour. 38 (2): 3, 147, 1869.

Fisquetia ornata Gaud., Bot. Voy. La Bonite, Atlas pl. 5,

fig. $1,8,9$, n. d. = [1841].

$P$. ornatus Hort., Hort. Soc. London, Jour. Proc. \& Misc.

n. s. 1: 1, 1868.

P. Lindenii Warb., Engler's Pflanzenreich IV, 9: 88, 1900.

956. Pseudotsuga taxifolia (Lamb.) Britton, New York Acad.

Sci., Trans. 8: 74, 1889.

Pinus taxifolia Lamb., Descr. Genus Pinus 1: 51, pl. 33,

1803.

Pinus taxifolia Salisb., Prodr. Hort. Chapel Allerton 399,

1796.

Abies taxifolia Lamb. ex Poir. in Lam., Encyc. Méth. Bot.

6: $523,(1804)=[1805]$.

A. taxifolia Mus. ex Du Tour, Nouv. Dict. Hist. Nat. 20:

114, 1803.

A. taxifolia Desf., Tabl. École Bot. Mus. Nat. 206, 1804. 
A. Douglasii Hort. ex Loudon var. taxifolia Loudon, Arb.

Frut. Brit. 4: 2,319, fig. 2,231, 1838.

A. Menziezii Mirb., Paris Mus. Hist. Nat., Mém. 13: 63,

1825.

A. mucronata Raf., Atl. Jour. 1: 120, 1832.

A. Douglasii (Lamb.) Lindl., Penny Cycl. 1: 32, ill. 1833.

Pinus Douglasii Sabine ex D. Don in Lamb., Descr. Genus

Pinus, ed. 3 (8vo.) 2: unnumbered page following 145 ,

pl. [47], 1832.

Pseudotsuga Douglasii (Lindl.) Carr., Traité Gén. Conif., ed. 2, 256, 1867.

Pseudotsuga mucronata (Raf.) Sudw. in Holz., U. S. Natl.

Herb., Contr. 3: 266, 1895.

P seudotsuga vancouverensis Flous, Soc. d'Hist. Nat.

Toulouse, Bul. 66: 340, 1934; Lab. Forest. Toulouse,

Trav. tome 1, vol. 2, art. 6: 12, ill., 1934.

Pseudotsuga Menziesii (Mirb.) Franco, Soc. Broteriana

(Coimbra), Bol. II, 24: 74, 1950.

See Sudworth, U. S. Dept. Agric., Div. Forestry, Bul. 17: 23-24, 1898;

Sprague \& Greene, Kew Bul. Misc. Inf. 79-80, 1938;

Little, Am. Jour. Bot. 31: 594-595, 1944;

Soc. Am. Foresters, Taxon 4: 20, 1955;

Gleason, Rhodora 57: 332-335, 1955;

de Wit, Taxon 5: 5, 1956;

Krajina, Madroño 13: 265-267, 1956.

Stafleu, Taxon 5: 18-19, 38-39, 1956;

Ross, Taxon 5: 41-43, 1956;

Shinners, Taxon 5: 43-46, 1956.

957. Pritchardia affinis Beccari var. Holaphila Beccari, Bishop Mus., Mem. 8(1): 39, 1921.

$P$. affinis Beccari var. halophila Beccari, Bishop Mus.,

Mem. 8(1): 2, 10, 12, 19, 23, pl. 4, fig. A, 1921.

958. Ranunculus Pallash Schlecht. ex Benson, Am. Midl. Nat.

53: 255, 1955.

R. Pallasii Schlecht., Animad. Ranunc. 1: 15, pl. 2, 1819. 


\section{INDEX*}

Abies, 321

canadensis, 46

Douglasii, 956

taxifolia, 956

glaucescens, 504

Menziesii, 956

mucronata, 956

religiosa

glaucescens, 504

taxifolia, 956

Abrotanum, 85

Absinthium, 23

Absynthium, 23

Acacia

tetragonocarpa, 589

Acanthonitschkea, 669

Acanthosabal, 322

Acer

barbatum

Longii, 547

platylobum, 547

carolinianum, 304

floridanum

Longii, 547

platylobum, 547

rubrum, 304

saccharinum

columnare, 178

saccharophorum, 604

saccharum, 178

sacchatum, 604

stenocarpum, 304

Treleaseanum, 604

Wilsoni, 924

Wilsonii, 924

Acharospora

alboatra, 283

Achras

Zapota, 69

zapotilla, 69

Acinaria, 756

Aciphylla, 348
Acoeloraphe, 322

Acoelorraphe, 322

Aconitum

ambiguum, 307

fudjipedis, 879

kunasilense, 659

Napellus

alpinum, 307

ambiguum, 307

variegatum

Canarum, 812

typicum, 812

Acosta, 29

Acrostichum

platyneuros, 148

punctatum, 213

Actinocheita

filicina, 800

Adelia, 714

Bernardia, 830

Aecidium, 276

Gaurae, 113

Hyperici frondosi, 571

Violae, 165

Aërides, 656

Aerva

tomentosa, 588

Aeschynomene

viscidula, 680

Aesculus, 385

Aëtia, 743

Agardhiella, 212

Agaricales, 260

Agaricus

Persooniana, 252

Persoonii, 252

Agasta, 610, 705

Agataea, 705

Agatea, 705

Agathis, 705

Agati, 705

Agation, 705

* Names of genera and higher taxa are printed flush, those of species are indented once, and those of all subdivisions of species are indented twice. The number listed is the case number. 
Agavaceae, 365

Agaveae, 365

Agavoideae, 365

Agroelymus, 415

Adamsii, 177, 415

jamesensis, 177

anticostensis, 415

strictus, 175

Agropsammelymus, 176

Agropyron pauciflorum, 953

repens $\times$ Elymus mollis, 415

Agropyrum

junceum $\times$ Elymus arenarius, 175

pauciflorum, 953

strictum, 175

Agrostis, 823

Ahnfeltia

concinna, 209

Gigartinoides, 209

Aira, 75

Albizzia

calliandra, 325

Albocrustum, 290

Albugo, 216

Alchemilla

vulgaris, 563

Alcicornium, 162

Aletris

japonica, 329

Aleurites

triloba, 731

Aleurodiscus, 200

amorphus, 210

candidus, 513

grantii, 210

Aligera

californica, 552

macrocera, 552

Alisma

Dama sonium, 346

Alsophila polypodioides, 265

Alternanthera

achyranth., 928

Achyrantha, 928

Althaea

foliis simplicibus, 883

Amaranthus, 608

Amarantus, 608

Amarella quinquefida, 67

Amaryllis Bella dona, 813

Amblyanthera suaveolens, 926
Amida, 542

Ammiaceae, 541

Amorpha microphylla, 339

nana, 339

Amphoradenium haalilioanum, 431

Amsinckia

Lemmonii, 131

Anadryum humile, 789

Anapausia, 821

Anaphalis margaritacea, 143

Andreaea, 228

Andreaeaceae, 228

Andreaeales, 228

Andreoskia, 643

Androcryphia, 284

Andromeda, 125

Andropogon

amboinicus, 394

Andruris

elegans, 328

palawensis, 328

Andrzeiowskia, 643

Anelsonia, 715

Anemone

ludoviciana, 587

Nuttalliana, 587

patens

Nuttalliana, 587

Wolfgangiana, 587

quinquefolia

bifolia, 81

interior, 81

Anetia, 539

Anetium, 539

Angelandra, 10

Angervilla, 172

Anguria, 744

Annamocarya indochinensis, 184 sinensis, 184

Anneslea amazonica, 319

Anoda

ovata, 904

Anonymos

aquatica, 309

pudic., 32

rotundifolia, 84

Antennaria

margaritacea, 143

Anthoceras, 237 
Anthoceros, 237

Havaiensis, 423

hawaiensis, 423

megalospermus, 421

megalosporus, 421

Anthocerus, 237

Anthochloa, 729

Apera, 823

Apiaceae, 541

Apionema

obovatum, 888

Apium

Ammi, 407

crispum, 24

hortense, 24

latifolium, 24

latifolium, 24

leptophyllum, 407

Petroselinum, 24

sativum, 24

Aplodiscus, 532

Aplopappus, 118

Apocynum

androsaemifolium, 521

fol. androsaemi., 521

muscipulum, 521

syriacum, 510

Aponogeton

oblongus, 886

Aporosa, 776

Aporusa, 776

Arabis

laevigata

heterophylla, 57

missouriensis

Deamii, 57

viridis

Deamii, 57

heterophylla, 57

Arachis

hypogaea

microcarpa, 167

Aralia, 420

Ginseng, 374

Arctium

Lappa, 523

majus, 523

majus, 523

Arcyphyllum

Difforme, 824

Areca

Catechu, 921

cathecu, 921

Argyroxiphium sandwicense, 93
Arisarum

Libani, 940

Aristeae, 534

Aristeinae, 534

Arnica

alpina

Lessingii, 814

angustifolia

Lessingii, 814

Lessingii, 814

Porsildiorum, 814

Aronia

hybrida, 152

monstrosa, 152

Artemisia, 23, 85

cana, 665

columbiensis, 665

matricarioides, 854

Artocarpus, 100

Arum

Colocasia, 357

esculentum, 357

Asclepias

syriaca, 510

A sparagus

caule herbaceo, 47

Aspergillus, 70

mangini, 430

minor, 430

Aspidium

aristatum

coniifolium, 234

Coniifolium, 234

Dryopteris, 89

Filix mas, 954

goggilodus, 233

montanum, 507

platyphyllum, 280

scabriusculum, 222

simulatum, 606

spinulo sum

dilatatum, 300

Asplenium

barbadense, 459

dentatum, 459

nidus, 825

Nidus avis, 825

platyneuron, 148

Souchei, 150

Trich. dentatum, 459

Trichomanes ramosum, 597

viride, 597

viridi, 597

Aspris, 75 
Aster

arcticus, 425

Asa-Grayi, 140

Fendleri, 734

nemoralis, 64

Nuttallii

Fendleri, 734

pseudo-Asa-Grayi, 140

Asterella

Bolanderi, 248

Astragalus

albus, 826

dispermus, 706

dispermus, 706

Earlei, 882

hircina

indica, 826

Humboldtii, 882

mollissimus earlei, 882

Punjabicus, 826

Asystasia, 827

Atacciae

cristatae, 311

schizocapsae, 311

Athamanta

chinensis, 410

A thyrium angustipinna, 646 montanum, 507

Atropa

physalodes, 169

Avena, 576

fatua, 663

eufatua, 663

fatua, 663

Baccharis cundinamarcensis, 877

Baileya, 144

Balduina, 44

Baldwinia, 44

Balsamona, 432

Bamia, 134 Manihot, 134

Bammia, 134

Bankesia, 516

Banksea, 516

Banksia, 516

Baranda, 610

Barbarea

barbarea, 828

vulgaris, 828

Barbosella

crassifolia

minor, 614
Barleria, 381

Barringtonia, 610

Baryxylum inerme, 925

Beautempsia, 829

Bebbia, 124

Bellonia, 873

Belowia, 908

Benthamantha, 16

Berberis canadensis, 889 simplex, 61

Bergia capensis, 907

Bernardia

Bernardia, 830

carpinifolia, 830

dichotoma

Betula genuina, 830

Andrewsii, 375

caerulea, 142

caerulea-grandis, 766

coerulea, 142

hanenisiensis, 585

occidentalis $\times$ papyrifera, 375

papyrifera

Andrewsii, 375

Betulinum

hanenisiense, 585

Bicornes, 347

Bidens

waianaeensis, 772

waianensis, 772

Biotia, 331

Blechnum

occidentale, 9

orientale, 9

Blephara, 531

Blepharostoma trichophyllum, 443

Bletia

Tankervilliae, 629

Blumea

grandiflora, 885

somaliensis, 894

Bocoa, 788

Boehmeria, 683

Boerhavia, 538 diffusa, 822

Boldu, 710

Boldus, 710

Boletus igniarius, 294

Tulipiferae, 256

Borya, 713 
Bostrychia

distans, 676

harveyi

$$
\text { distans, } 676
$$

Botryopsis, 610

Boudiera, 253

Brachycome leptocarpa, 451 tenuiscapa, 326 uliginosa, 451

Brachy corythoides, 586

Brachyramphus

lactucoides, 930

Bradleia, 736

Bradleja, 736

Brassia, 133

Braussonetia papyrifera, 947

Brittonamra, 16

Brodiaea Orcuttii, 13

Brosimum parinarioides, 112

Broussaisia arguta arguta, 315

Broussonetia papyrifera, 947 secundiflora, 947

Brugmansia arborea, 506

Brunfelsia mire, 831

Bryum argenteum, 185

Bucklandia, 65

Buddlea, 439

Buddleia, 439

Buddleja, 439

Buddleya, 439

Budlaea, 439

Budlea, 439

Budleia, 439

Budleja, 439

Bulboschoenus macrostachyus, 522

Bulgaria, 279 globosa, 279 inquinans, 279

Bupariti, 878

Butonica, 610

Byssus

botryoides, 263

Cactus, 153

ferox, 153
Caesalpinia

ferruginea, 925

inermis, 925

Cakile edentula, 582

lanceolata, 582

Caladium, 80 acre, 80 Colocasia, 357 esculentum, 357

Calamagrostis ophitidis, 645

Calceolaria Storkii, 810

Callicarpa resinosa, 342

Calliopsis tinctoria atropurpurea, 754

Calucechinus, 159

Calusparassus, 159

Calvatia, 239

Calystegia sepium rosea, 5

Cambogia Gutta, 687

Camellia sinensis, 535 Thea, 535

Camirium, 731

Campanula caulibus diffusis, 722

Canarium commune, 570 indicum, 570 vulgare, 570

Canavalia galeata, 786 pubescens, 126 microcarpa, 569 pubescens, 126 turgida, 569

Candida, 441

Canthium barbatum australense, 811 tubuai, 811

Capparis cordifolia, 885 cynophallophora, 816 flexuosa, 816 Jamaicensis, 816

Capraella, 220

Capura, 460 
Cardamine

digitata, 398

hyperborea, 398

Matthioli, 108

Richardsonii, 398

\section{Carex}

chikungana, 767

chlorantha, 114

chlorocystis, 107

Collinsii, 6

Idahoa, 34

katahdinensis, 139

littoralis, 438

Muhlenbergii, 110

mund

mundaeformis, 899

wahuensis, 579

Carpentaria, 320

Carpenteria, 320

Carpentiera, 320

Carpoceras, 237

Carpoceros, 237

Carpolithes compositus, 149

Carya, 302

illinoënsis, 301 sinensis, 184

Caryopitys monophylla, 801

Caryotaxus, 325

Cassia

Chamaecrista, 619

hebecarpa, 51

nictitans

hebecarpa, 51

Castalia, 531

Leibergi, 723

Catharanthus

roseus, 644

albus, 644

Ceanothus

Americanus, 677

Cedrella

Mahag., 949

Centauriom

Centaurium, 68

Centaurium

Erythraea, 68

minus, 68

umbellatum, 68

Ceodes

umbellata, 11

Umbellifera, 11

Cephalanthus

occidentalis

lanceolatus, 755
Ceramium

affine originale, 653

Ceranthus, 237

Ceratobasidium atratum, 458 plumbeum, 458

Cerbera maculata, 603 parviflora, 603

Cercocarpus alnifolius, 712 betula efolius Blancheae, 712

betuloides, 712 alnifolius, 712 blanchae, 712

montanus blanchae, 712 glaber, 712

Cercospora mori, 652 moricola, 652 morina, 652 platyspora, 452

Ceriosporopsis halima, 299

Cestichis Yamadae, 66

Cestrum

Weberbauerei, 616

Chaerophyllum

Tainturieri

floridanum, 685

Chatyllis, 240

Chayota, 919

Chenopodium oahuense, 579

Chironia Centaurium, 68

Chlorococcum, 263

Chocho, 919

Chonemorpha penangensis, 549

Chrysonephos, 288

Chrysophaeum, 288

Chrysothamnus, 132

Ciborinia

bifrons, 518

confundens, 518

Whetzelii, 518

Cibotium

glaucum

Nealiae, 223

Nealiae, 223 
Cicuta

maculata, 7

Cinchona, 544

Circaea

intermedia, 33

latifoli a, 33

lutetiana

canadensis, 33

quadrisulcata, 33

Maximowiczii, 33

mollis

Maximowiczii, 33

quadrisulcata

canadensis, 33

Citharexylum

spinosum, 914

Citrullus, 744

vulgaris, 744

Cladium, 528

angustifolium, 632

Cladonia

furcata

subrangiformis, 251

subrangiformis, 251

Cladophora

Aegagropila

Sibogae, 243

Sibogae, 243

Cladophoropsis, 203

Clavaria, 42

Claytonia

depressa

latifolia, 316

Washingtoniana, 63

Clematis

Missouriensis, 529

pseudoscabiosa efolia normalis, 832

virginiana

missouriensis, 529

Clermontia

Kakeana, 686

montis-Loa, 762

Clerodendron

aculeatum gracile, 76

canescens, 781

Clerodendrum

aculeatum

gracile, 76

capitatum

vanderysti, 939

Cloëzia, 809

Clypeothecium

Weirii, 833
Cnidone

Mentzeloides, 929

Cochliospermum, 908

Cococynthis, 744

Cocos

nucifera, 138

Codia, 79

Coffea

Kaduana, 888

Colea

hispidissima, 188

Collinsia, 6

Collinsiae, 6

Collinsonia, 6

Colocasia

acris, 80

Antiquorum, 357

esculenta, 357

esculenta, 357

acris, 80

typica, 357

Columellia, 863

Conioselinum

canadense, 410

chinense, 410

Conomorpha

roraimae, 572

Convolvulus

acetosaefolius, 537

grandiflorus, 18

littoralis, 537

sepium

Americanus, 5

stoloniferus, 537

tiliaefolius, 18

Conyza

Japonica, 369

Conyzopsis, 59

Coprosma, 615

ochracea

kaalae, 404

Corallina

barbata, 540

Cordyceps, 282

Cordylia, 282

Cordyliae, 282

Cordylophorum, 82

Coreopsis

tinctoria, 754

atropurpurea, 754

tinctoria, 754

Cornutia corymbosa, 916

Corticium amorphum, 210

atratum, 458 
Corydalis

aurea, 366

aurea, 366

typica, 366

Corynocarpus, 380

Corypta, 237

Cotinus

americanus, 648

obovatus, 648

Cotula

alba, 618

Cotyledon, 35

Cracca, 16

Crataegus

glabana, 935

pexa, 935

senta, 935

Crataeua, 872

Crataeva, 872

Crateva, 872

Creonectria coccinea, 625

Crepis

Froelichiana, 561

Crescentia, 834

Cujete, 906

Crotalaria rotundifolia, 84

Crucita hispanica, 673

Cruzita hispanica, 673

Crypsinus, 129

Cucurbita, 835

Citrullus, 744

Cuiete, 906

Culhamia, 183

Cuphea, 432

Cuscuta africana, 666

Cyanea

Bishopii, 154

Bonita, 154

Kunthiana, 154

Cyathea montana, 507

Cyathodes

Banksii, 760

imbricata, 612

Tameiameiae, 785

Cyclomycetella pavonia, 840

Cycloporellus iodinus, 840

Cyclosorus gongylodes, 233
Cyclospermum

leptophyllum, 407

Cydonia

Cydonia, 721

Oblonga, 721

vulgaris, 721

Cymopolia

Barbata, 540

Rosarium, 540

Cynodon

Dactylon, 841

Cyperus

Ferax, 815

Kyllingia, 318

odoratus, 815

Papyrus, 842

phleoides, 602

Cyphella, 200

amorpha, 210

Cyphokentia

samoensis, 818

Cyrtandra, 681, 782

Clarkei

ovatifolia, 791

grandifolia, 87

Hosakae, 400

kalichii

tristis, 941

laxiflora

grandifolia, 87

rhizantha, 87

Lessoniana, 368, 403

Lessoriana, 403

Lilianae, 386

Nadeaudi, 869

olona, 843

rhizantha, 87

stupantha, 87

tahitensis, 869

Taitensis, 869

triflora

grandifolia, 87

velutina, 768

Wilderi, 368

Cyrtophyllum

lanceolatum, 7

Cystopteris

bulbifera, 293

fragilis simulans, 719

montana, 507

Cystopus, 216

Daboecieae, 60

Dactilon, 841

Dactylon, 841 
Daedalacanthus nervosus, 913

Dalea enneandra, 901 laxiflora, 901

Dalechampia, 360

Damasonium Alisma, 346 dama sonium, 346 Stellatum, 346

Danthonia spicata pinetorum, 763 thermale, 763

Dasiphora riparia, 927

Davallia hirta, 270

Davyella, 729

Debregeasia, 732

Delisea, 20

Delissea, 20 Kunthiana, 154

Delitschia, 20

Delphinium elatum occidentale, 796

Nuttallianum, 88 Nuttallii, 88 occidentale, 796

Dendrophthoë, 187

Dendrophthora Willtreleasii, 442

Dendrophtoe pentandra, 187

Dentaria digitata, 398

Deschampsia, 75

Desmocephalum, 675

Desmos chinensis, 790 cochinchinensis, 790

Destrugesia, 844

Destruguezia, 844

Diaporthe Euporthe, 241 picea, 241

Dicaeoma

Polygoni-amphibii, 359

Violae, 165

Dicarphus rubens, 654

Dicranum yakushimense, 158

Dictyocalyx, 581
Dictyota variegata, 779

Didymella Ilicis, 845

Didymosphaeria Equiseti-hiemalis, 720

Diellia

Brownii, 92

Dilphinium peregrinum, 622

Diodia teres, 609

Dioscorea alata, 43 bulbifera sativa, 413 sativa, 413

Dipetalon, 432

Diplacorchis, 586

Diplodina persicae, 281

Diplomorpha, 460 elongata recurva, 460

Diplopappus, 118

Diplosporis, 277

Discovium, 600

Docanthe alba, 917

Dolichos catjang, 639 galeatus, 786

Dondia, 908

Doxomma, 610

Draba Crockeri, 546

Drosera anglica, 891 intermedia, 891 longifolia, 891

Dryandra, 731

Dryobalanops aromatica, 514

Dryopteris, 8 disjuncta, 89 filix mas, 954 goggilodus, 233 gongylodes, 233 Linnaeana, 89 disjuncta, 89 ornata, 265 pulchella, 89 scabriuscula, 222 simulata, 606 spinulosa dilatata, 300 triangularis, 89 
Drypetes

formosana, 336

Dubautia, 128

raillardioides, 509

Railliardioides, 509

Rockii, 717

scabra, 765

Duckera, 915

Dumortiera

hirsuta, 232

latior, 232

nepalensis, 232

Durio, 96

Duvernaya, 432

Dymczewiczia, 146

Ebenopsis, 400

Echites

difformis, 592

Echites, 933

laxa, 926

puberula, 592

suaveolens, 926

Eclipta

alba, 618

erecta, 618

prostrata, 618

Ectocarpus

Duchassaingianus, 235

Eggelingia, 358

Eichornia, 922

Elaeagnus

argentea, 105

argenteus, 105

commutata, 105

Elaeagrus, 105 argentea, 105

Elaeococca, 731

Elaphoglossum reticulatum, 672

Elatostemma Pedunculatum, 635

Eleocharis, 109

capitata, 846

Elizabethia miniata, 221

Elsbolzia, 19

Elschotzia, 19

Elsholtia, 19

Elsholtzia, 19

Elsholzia, 19

Elshotzia, 19

Elssholzia, 19

Elymopyrum, 415

Elymotrigia, 156
Elymus

$$
\begin{gathered}
\text { ampliculmis, } 370 \\
\text { arenarius, } 370 \\
\text { mollis, } 370 \\
\text { villosus, } 370 \\
\text { capitatus, } 370 \\
\text { cinereus, 638 } \\
\text { condensatus } \\
\text { pubens, 638 } \\
\text { dives, 370 } \\
\text { mollis, 370, } 415 \\
\text { pauciflorus, 953 } \\
\text { trachycaulus, } 953 \\
\text { Engelmannia, } 10 \\
\text { Englemannia, } 10 \\
\text { Enteromorpha } \\
\text { compressa } \\
\text { constricta, } 262
\end{gathered}
$$

Epichloe, 250

Epichloë, 250

Epidendrum, 401

Epipremnum

humile, 789

Equisetum

campanulatum, 230 scabrum, 230

maximum, 601

ramosissimum, 230 scabrum, 230

sylvaticum neoserotinum, 179 pauciramosum, 179

Telema teia, 601

Eragrostis

Hosakai, 560

perplexa, 181

wahowensis, 579

Eranthemum

nervosum, 913

pulchellum, 913

Erica

caffra, 580

Erigeron

Japonicum, 369

Eriosorus, 408

Erysimum

Barbarea, 828

Erythraea

Centaurium, 68

Erythrobarbula, 201

Erythrophyllum, 201

Eschholtzia, 19

Eschholzia, 19

Escholtzia, 19

Eschscholtzia, 19 
Eschscholzia, 19

Esculus, 385

Espeletia amplexicaulis, 14

Etballia, 788

Euartemisia, 85

Euarthronia, 615

Eucanna, 54

Eucuscuta, 367

Eu-Diplacorchis, 586

Eugenia hawaiiensis, 769 parvifolia, 397 rariflora parvifolia, 397 waianensis, 397

Euodia, 387

Euonymus, 25

Eupatorium

Benjamin-Lincolnii, 892

purpureum, 726

verticillatum, 726

Euphiladelphus, 596

Euphorbia

Celastroides

Odonatoides, 871

maculata, 746

pseud-nutans, 893

supina, 746

Eupritchardia, 545

Euptelea, 136

Eurotium, 70

herbariorum minor, 430

minor, 430

Euryale amazonica, 319

Eurynome, 615

Eusabatia, 454

Eustrephus

angustifolius, 40

latifolius

angustifolius, 40

timorensis, 17

Euzodiomyces, 244

Evodea, 387

Evodia, 387

Evonymus, 25

Fagara

kauaiense, 45

kohuana, 45

Fagaster, 159

Dombeyi, 174
Festuca

Halleri, 937

Fevillea, 658

Ficus

bengalensis, 704

benghalensis, 704

Beniamina, 709

benjamina, 709 nuda, 709

nuda, 709

velascoi, 373

vilamilii, 752

Filago, 336

Filicula, 507

bulbifera, 293

montana, 507

Filix

montana, 507

pumila, 89

Fimbriaria

Bolanderi, 248

Fimbristylis

cymosa

pycnocephala, 803

marguesana, 49

marquesana, 49

pycnocephala, 803

Fisquetia

ornata, 955

Fissenia

Capensis, 929

mentzeloides, 929

Flammula, 224

Foetaxus, 326

Fomes

arctostaphyli, 294

igniarius, 294

Forestiera, 714

Forsellesia, 808

Forsteronia

difformis, 592

Fouilloya, 355

Fraxinus

caroliniana, 74

expansa, 74

juglandifolia, 74

lanceolata, 74

pennsylvanica

lanceolata, 74

subintegerrima, 74

pubescens

subpubescens, 74

viridis, 74

Frullania

helleri, 332 
Fucus

digitatus, 269

hyperboreus, 269

scoparius, 269

Fumago, 218

Fumaria

aurea, 366

Fusicladium

depressum, 452

Fusicoccum, 943

Gahnia

gahnia eformis, 520

Gaudichaudii, 520

Gal apagoa, 426

Galega, 16

Garcinia

c ambogia, 687

Gardenia

Remyi, 312

Gaultheria, 255

Gaultiera, 255

Gaura

mollis, 116

Gautiera, 255

Gautieria, 255

Geigeria

plumosa, 564

Geitonoplesium

cymosum timorense, 17

Gelidium, 42

lanceolatum, 227

samoense

lineare, 505

minus, 505

Geminispora, 277

Geniostoma, 97

Cyrtandrae, 758

Gentiana

Amarella, 67

Centaurium, 68

Gerbera

Conrathii, 163

viridifolia

Conrathii, 163

Gertrudia, 298

Getonia, 782

Gifola, 336

Gilia

$$
\text { Parryae, } 122
$$

Gillespiea, 938

Glaucae, 54

Globosopyreno, 292

Glochidion, 736
Glochidionopsis, 736

Glochisandra, 736

Gloniopsis

Ellisii, 361 smilacis, 361

Glossopetalon, 808

Glossopetalum, 808

Gmelina

arborea, 633

Gnaphalium

margaritaceum, 143

sandwicensium, 161

canum, 161

olivaceum, 161

typicum, 161

Goniolithon, 215

Goniophlebium, 818

Gonolobus

jamaicensis, 636

rostratus, 636

Gouania

Hillebrandi, 900

Gouldia

terminalis

antiqua, 898

eukaala, 667

Hosakai, 668

kaala, 667

kauensis, 898

Grammitis

subpinnatifidum, 431

Graya, 55

Grayia, 55

Greyia, 55

Greyiaceae, 55

Greyieae, 55

Grifola

Tuckahoe, 945

Gualteria, 255

Guesmelia, 526

Gymnobythus, 83

Gymnocarpum

Dryopteris, 89

disjunctum, 89

Gymnopilus, 224

Gymnosorus, 229

Gynoon, 736

Gynoxys

columbian, 424

columbiana, 424

Macfrancisci, 565

sancti-antonii, 751

Habenaria

straminea, 27 
Haliclona oculata, 41

Halymenia

Durvillaei denudata, 207 edentata, 849 formosa, 797 formosa, 797

Haplopappus, 118

Harrimanella, 125

Havardia, 400

Hedyotis rosea, 12

Hedysarum boreale cinerascens, 352

cinerascens, 352

Mackenzii, 352 canescens, 352

Macquenzii canescens, 352

Heleocharis, 109 kasakstanica, 515

Helicoma salinum, 299

Heliotropium anomalum, 911 incanum, 911

Helotium, 259 brassicaecolum, 259

Hemerocallis flava, 503 japonica, 329 lancifolia, 329 Lilio Asphodelus, 503

Hemitrema, 220

Heracleum lanatum, 511 maximum, 511

Herberta, 285

Herbertus, 285

Hernandia, 303 labyrinthica, 837

Hernandria, 303

Herpophyllon, 623

Herpophyllum, 623

Herverus, 285

Heterachaena massauensis, 930

Heterobasidion, 749

Heterobasidium, 749

Heteropogon, 99

Heterosporium tshawytschae, 568
Hibiscus

caerulescens

Humbertiellus, 850

mandrarensis, 851

Hickorius, 302

Hicoria

Pecan, 302

Hicorius

Pecan, 302

Hippocratea

Urceolus xerophila, 688

Hoehneliogaster, 258

Hönelogaster, 258

Holcus, 349

Hookera

multipedunculata, 13

Orcuttii, 13

Horkelia, 383

Horsfieldia, 383

Hosta

japonica, 329

lancifolia, 329

Houstonia

patens

pusilla, 12

pusilla, 12

rosea, 12

pygmaea, 12

Taylorae, 12

Hoya

diversifolia, 502

esculenta, 502

orbiculata, 502

Humbertia, 559

Humbertiella, 559

Pseudohenrici, 850

Humbertochloa, 559

Hutchina, 951

Hutchinsia, 951

Hutchintia, 951

Hutschinsia, 951

Hydrocotyle cordata, 309

Hygropyla nepalensis, 232

Hymenophyllum polyanthum, 264

Hypnomonas pleiopyrenigerum, 422

Hypnum

gracile lancastriense, 946

Stereodon

virginianus, 444

torquatum, 297 
Hypochaeris, 37

Hypochoeris, 37

Hypocreopsis solidus, 590

Hypoderma smilacis, 361

Hypodermopsis smilacis, 361

Hypoxylon solidum, 590

Hyssopus, 123

Hysterium smilacis, 361

Hysterographium smilacis, 361

Icica

Timoriensis, 2

Idahoa

scapigera, 34

Ifloga, 336

Ilex

Curitibensis, 881

Mate, 881

Paraguariensis, 881 genuina, 881

Inga

pterocarpa, 925

urabensis, 804

Inocarpus, 788

fagiferus, 788

Inodaphnis, 788

Ipomaeella, 918

Ipomoea

acetosaefolia, 537

carnosa, 537

grandiflora, 18

littoralis, 537

stolonifera, 537

Iresine

celosia

lutescens, 305

Irpex

tulipifera, 256

Isotoma

longiflora

runcinata, 445

runcinata, 445

Itajahya, 295

Iuglans

illinoiensis, 302

Ixora

nigritans, 735

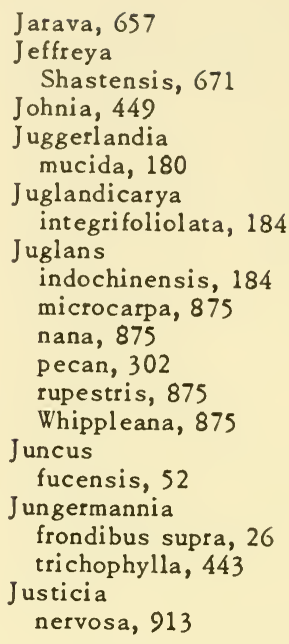

Kingdon-Wardia, 30

Kissenia

sp athul ata, 929

Klibea, 341

Kommamyce, 419

Konig, 936

Koniga, 936

Korthal sella striata, 551

Kosaria, 435

Kosteletzkya, 127

Kuhnioides, 124

Kyllinga monocephala, 318 nemoralis, 318

Kyrtandra, 681, 782 aristata, 681

Labiaceae, 651

Labiatae, 651

Labl ab microcarpus, 569

Labordia Cyrtandrae, 758

Fagraeoidea glabrescens, 574

Lactuca sessilis, 574

massaviensis, 930

Lagenaria, 835

Lagenifera, 852 
Lagenofera, 852

Lamiaceae, 651

Laminaria

digitata, 269

hyperborea, 269

scoparia, 269

Lampocarya Gaudichaudii, 520

Langermannia, 239

Langeveldia acuminata, 635

Lantan a arubensis, 637

Lappa communis major, 523 major, 523 vulgaris, 523

Larix laricina, 377

Laserpitium A ciphylla, 348

Lastraea Dryopteris, 89 scabriuscula, 222 simulata, 606

Lathyrus japonicus

Laurus glaber, 395 albida, 391

Sassafras, 391 Variifolia, 391

Lecanora raroia, 271

Leiacina, 853

Lenormandia, 261

Lepachys columnaris, 53

Lepidium lasiocarpum, 647 O-Waihiense, 579

Lepidococcus, 923

Lepimenes, 367

Leptodontium humillimum, 249

Lepturus reptans, 31

Lerchea, 908

Le squer ella, 600

Leucanthemum, 405

Leucocnide, 732

Leuconymphaea, 531
Leucorchis

albida, 27

straminea, 27

Leveillea, 557

Liabum columbianum, 424

Lievena, 526

Limnanthes gracilis, 702 gracilis, 702

Limodorum Incarvillei, 629

Tancarvilleae, 629

Tankervilliae, 629

Lindera laxiflora, 897 Tzumu, 897

Liparis Yamadae, 66

Lipochaeta, 168 lobata

denticulata, 775

Lipotriche, 168

Lit anum, 577

Lithocarpus Rodgerianus, 605

Lithophyllum, 287

Lithospermum incanum, 911

Lit sea laxiflora, 897

Lobelia affinis, 617

Gaudichaudii, 711 coccinea, 711 typica, 711

glandulosa obtusifolia, 86 lanceol ata, 73 macrostachys, 730 puberula gl abella, 86 obtusifolia, 86 succulenta, 617 zeylanica, 617

Lobocarpus, 736

Lochnera rosea, 644

Lofgia, 336

Logfia, 336

Lophianthus calycinus, 364

Lophiocarpus, 364 
Lophodium

Filix-mas, 954

Lophostigma, 453

Lophotocarpus calycinus, 364

Loranthus longiflorus, 187 pent andrus, 187

Luehea, 558

Luhea, 558

Lupinus alpicol a, 770

Hellerae, 362

Helleri, 362

minimus

Hellerae, 362

Lupulus

orbicul aris

Hill ebrandtii, 900

Luzuriaga

cymosa, 17

Lycopodium

alopecuroides

adpressum, 219

annotinum

proliferum, 225

spica omacio, 225

chamaecyparissus, 778

complanatum

triceps, 778

ecuadoricum, 701

erubescens, 231

Hamiltonii, 208

heterophyllum, 553

in undatum

appressum, 219

venustulum 553

\section{Macaranga}

Maudslayi, 324

quadrigl andulosa variablis, 741

salomonensis, 773

Maerua

oblongifolia

pallida, 920

Mahonia simplex, 61

Malaxis, 313

Malus ioensis, 621

Mammea, 607

Mammillaria, 153
Mandevilla

laxa, 926

suaveolens, 926

Tweediana, 926

Margyricarpus, 562

Mariscus, 528

phleoides, 602

Marqui sia, 615

Marsilea, 214

Martensia, 220

Matourea, 172

Matricaria

matricarioides, 854

Matricarioides, 854

Matteucia

pensylvanica, 217

Struthiopteris, 217

Megadendron, 610

Mel al euca

Leucadendron, 429

Leucodendra, 429

Melalevca

Leucadendra, 429

Mel anocarpae, 915

Melica

Nebrodensis, 139

Melicocca, 372

Melicoccus, 372

Meliola forbesii, 855 gregoriana, 855

Melobesia membranacea, 716

Melochia concatenata, 390 corchorifolia, 390

Menichea, 610

Mentha

arvensis $\times$ viridis, 78

bracteolata, 737

gentilis, 78

Wirtgeniana, 78

Menthaceae, 651

Meranthrepta pinetorum, 763 thermale, 763

Mercurialis perennis, 748 spicata, 748

te sticulata, 748

Merisma candida, 513

Mertensia, 220

Mesotrema, 220 
Met eorus, 610

Metrosideros collina

glaberrima, 533

imbricata, 519

polymorpha, 519

lutea, 343

polymorpha

imbricata, 519

Micranthemum, 111

Microchaeta, 168

Microoecia, 909

Microsorium

punctatum, 213

Microstylis, 313

Microterus, 129

Migandra, 428

Mikania stereolepis, 650

Mimosa

Catechu, 921

Mitraria, 610

Monarda

punctata

villicaulis, 876

Monophyllaea, 135

Montia

latifolia, 316

Washingtoniana, 63

Morelotia gahni aeformis, 520

Morocarpus, 732

Moutabea, 29

Musa

Fehi, 512

paradi siaca

troglodytarum, 512

sapi entum, 512

Troglodylarum, 512

Troglody tarum, 512

Uranoscopos, 512

Uranoscopus, 512

Musaenda

fr fondoso, 750

frondosa, 750

Muscites, 151

florissanti, 151

Muscus

marinus, 186

Mycosph aerella

elymifoliae, 242

Myroxylon, 626

Myrtus

Leucadendra, 429
Naegelia, 725

Nägelia, 725

Nama

sandvicense, 95

Sandwicensis, 95

Nani, 343

Nania, 343

lutea, 343

polymorpha

glaberrima, 533

Narda, 598

Nardia, 598

Nardius, 598

Nardus, 598

Nectria

coccinea, 625

cytisporina, 236

ditissima, 625

Nelumbium, 39, 531

luteum, 39

Nelumbo, 39, 531

lutea, 39

pentapetala, 39

Nemacladus

pinnatifidus, 771

ramosissimus

pinnatifidus, 771

Nenuphar, 531

Neostapfia, 729

Neo-Urbania, 611

Nepenthes mirabilis, 4 Phyllamphora, 4

Nephrodium, 8

dilatatum, 300

Dryopteris, 89

Filix mas, 954

scabriusculum, 222

simula tum, 606

Neraudia, 628

Neyraudia, 628

Nicandra

Physalodes, 169

Phy saloides, 169

Nigredo

Hyperici-frondosi, 571

Nitella, 756

Nodularia

balsamicola, 210

Nomosa, 160

Nostoc, 447

Botryoides, 263

Nostoch, 447

Noteroclada, 284 
Nothofagus, 159

Notonia, 449

Nototrichium sandwicense

Forbesii, 524

Nufar, 531

Nummularia, 419

Nuphar, 531

Nymphaea, 531

Leibergi, 723

Nelumbo, 39

pentapetala, 39

Victoria, 319

Nymphea, 531

Nymphodes

aquaticum, 309

Nymphoides, 309

Nymphona, 531

Nymphosanthus, 531

Nymphozanthus, 531

Obeliscaria columnaris, 53

Obelisteca pinnata, 53

Ochrosia

borbonica, 603, 934

elliptica, 603

maculata, 603, 934

noumeensis, 603

parviflora, 603

platy spermos, 934

undulata, 934

Odontoglossum aureo-purpureum, 777 a uropurpureum, 777

Odostemon simplex, 61

Oecidium, 276

Oedogonium

Kurzii ovatum, 409

Oenothera argillicola, 792

Hookeri eu-Hookeri, 314

Oesculus, 385

Oglifa, 336

Onagra argillicola, 792

Onobrychis ralphii, 826

Onoclea

Struthiopteris, 217
Ophiolobus

glomus, 649

Opuntia

missouriensis, 153

poly acantha, 153

Orthopogon

abortivus, 733

Orthosorus, 229

Ory zop si s, 101

Osmunda

Struthiopteris, 217

Owenia

cerasifera, 2

Oxalis

W'rightii, 345

Oxytropis

campestris johannensis, 139 johannensis, 139

Pachypodium, 856

Padina

Howeana, 779

variegata, 779

Padus

eximia, 584

serotina

eximia, 584

Paeonia

albiflora, 713

edulis, 713

Reevesian a, 713

lactiflora, 713

Reevesiana, 713

Pallavicinius, 285

Palmocarpon, 149

compositum, 149

Panax

Ginseng, 374

Schin-seng, 374

Pandanus

aimirikiiensis, 173

Ala-Kai, 627

aruensis, 591

contracta, 591

contractus, 591

fanningen sis, 354

Hermsianus, 354

ornatus, 955

platiphyllus, 932

platyphyllus, 932

saipanensis, 455

spiralis, 354

spurius, 450

Pandorea, 728 
Panicum

abortivum, 733

affine, 912

a sperum, 733

ciliatiflorum, 141

ciliatifolium, 141

Colliei, 912

dactylon, 841

lanaiense, 912

Mattamusketense, 382

paludosum, 780

spine scens, 733

Papa, 285

Parechtites

Thunbergii, 592

Parnassia,

Turczaninowii, 147

Pasania

Rodgeriana, 605

Paspalum

setaceum calvescens, 91

Passiflora

edulis

flavicarpa, 77

mariquiten sis, 910

Patamogeton, 38

Pauahia sideroxyli, 238

Paurotis, 322

Pavia, 739

Peckifungus, 440

Pelaphia, 615

Pelea brownii, 28

Pellaea ternifolia, 903

Pellicularia, 247

Peltophorum ferrugineum, 925 inerme, 925

pterocarpum, 925

Roxburghii, 925

Penaea, 130

Penecillium frequetans, 202

Peniophora admirabilis, 508

affinis, 747

laevis, 747

ludoviciana, 245

Pentagonia

physalodes, 169
Pentaphylloides

arbu scula, 927

fruticosa, 927

Pentaptera

mollis, 306

Pepo, 835

Peredinium

claudicanoides, 757

depressa, 204

depressum, 204

divergens, 204

reniforme, 204

marinum, 204

Periphericae, 281

Perispermon, 784

Perispermum, 784

Perrierophytum

Humbertianthus, 850

Petrophiloides, 378

Petroselinum

crispum, 24

hortense, 24

Petroselinum, 24

sativum, 24

Peziza

amorpha, 210

Pfeifferago, 79

Phaeobul garia

inquinans, 279

Phaius, 857

Phajus, 857

Tankervillii, 629

Phaseolus

acutifolius, 550

cylindricus, 639

Phegopteris

Dryopteris, 89

disjuncta, 89

Philacra

auriculata, 753

Philadelphus

nepalensis, 952

oreganus, 948

triflorus, 952

Phl eum

intermedium, 3

nodosum, 3

pratense, 3, 660

vulgare, 660

vulgare, 660

Phlox

carolina

heterophylla, 530

heterophylla, 530 
Phoenix amboinica montana, 394

Phylanthera, 555

Phyllamphora mirabilis, 4

Phyllanthera, 555

Phyllites, 344 dentatus, 344 kaiwakaensis, 448

Phyllostegia parviflora, 764

Gaudichaudii, 764

Phymatopsis, 129

Physalodes, 169

physalodes, 169

Physaloides, 169

Piaropus, 922

Picea

canadensis, 46

glauca, 46

glaucescens, 504

Sitchensis, 335

Picria, 624

Pimelia, 516

Pimpinella

leptophylla, 407

Pinus

attenuata, 548

australis, 310

ball, 72

balli, 72

baumani, 353

bowm an ii, 353

cembrodes

monophylla, 801

Douglasii, 956

glauca, 46

Laricina, 377

Menziesii, 335

monophyllus, 801

Murrayana, 121

palustris, 310

resinosa, 137

sitchensis, 335

taxifolia, 956

tuberculata, 548

Pisonia

umbellata, 11

Pisum

maritimum glabrum, 395

Pithecellobium, 400 monopterum, 456

Pithecollobium, 400

Platycarya, 378

Platycerium, 162
Platy schkuhri a, 82

Platyspermum, 34

Platy zomataceae, 363

Plectocomiopsis dubius, 371

Plectritis californica, 552

macrocera, 552

Pleiogynium

Sol andri, 2

timoriense, 2

Pleuranthodes

hillebrandii, 900

Hillebrandit, 900

Pleuropetalon, 583

Pleuropetalum, 583

Pluchea

Poa

somaliensis, 894

amboinica, 394

Pocockiella, 229

Poeonia albiflora, 713

Poinciana

Roxburghii, 925

Polemonium

caeruleum occidentale, 836 occidentale, 836

Polygala verticillata, 839

Polygonum convolvuloides, 267 pterocarpum, 267

Polypodium, 98 bulbiferum, 293

dilatatum, 300

Dryopteris, 89, 858 disjunctum, 89

filix mas, 954

Haalilioanum, 431

montanum, 507

ornatum, 265

pulchellum, 89

punctatum, 213

Robertianum, 858

subpinnatifidum, 431

virginianum, 145

vulgare, 145 acuminatum, 817

Polyporus

Fijii, 291

igniarius, 294

iodinus, 840

striatus, 840

tulipiferus, 256 
Polystichum

aristatum

Voniifolium, 234

Dryopteris, 89

Felix mas, 954

Polytrichum

florissanti, 151

Portulaca

Portulacastrum, 56

Portulacastrum, 56

Potamogeton, 38

Purshii, 351

Potamogetum, 38

Potamogiton, 38

Potentilla

arbuscula, 927

floribunda, 927

fruticosa, 927

villosissima, 927

Loureironis, 927

pensylvanica, 890

Pothuava, 859

Prasium parviflorum, 764

Premna

arborea, 633

corymbosa, 916

integrifolia, 916

obtusifolia, 916

serratifolia, 916

tahitensis, 633

Taitensis, 633

Prionitis, 227

lanceolata, 227

linearis, 227

Pritchardia, 545

affinis

halophila, 957

Holaphila, 957

Pritchardioxylon, 545

Procris

acuminata, 635

Protococcus

botryoides, 263

Prunus

eximia, 584

serotina

eximia, 584

Prunygdalus hybrida, 594

Przewalskia, 543

Pseudendoclonium arctica, 268

Pseudoraphis a spera, 733 spinescens, 733
Pseudosassafras

laxiflora, 897

Tzumu, 897

Pseudo solidum, 590

Pseudotsuga

Douglasii, 956

glaucescens, 504

Menziesii, 956

mucronata, 956

taxifolia, 956

glauca, 504

glaucescens, 504

vancouverensis, 956

Psychotria

Taupotinii, 115

Pteretis

pensilvanica, 217

Pteridanetium, 539

Pteridium, 703

Pterigium

costatum, 514

Pteris

ternifolia, 903

Pteronevron, 661

Pterula

brunneosetosa, 794

Puccinia, 246

Convolvuli, 15

densa, 165

Polygoni, 359

amphibii, 359

violae, 165

Pulsatilla

hir sutis sima, 587

ludoviciana, 587

patens, 587

Wolfgangiana, 587

Putranjiva formosana, 338

Pvccinia, 246

Pycnosphace, 82

Pyrus

americana, 886

coronaria

Ioensis, 621

Cydonia, 721

hybrida, 152

Ioensis, 621

microcarpa, 886

Quercinum

anataiense, 678

Quercus

anataien sis, 678

borealis, 745

maxima, 745 


\section{(Quercus)}

Lowilliamsii, 399

Margaretta, 396

marilandica, 36

marylandica, 36

minor

Margaretta, 396

mohavensis, 344

rubra, 745

borealis, 745

stellata

Margaretta, 396

virginiana

eximea, 806

eximia, 806

Quesnelia, 526

Racioborskiomyces, 240

Radermachia, 100

Rafinesquia, 308

Raillardia, 128 rocki, 717

Railliardia, 128, 509

Rockii, 717

scabra, 765

hispidula, 765

Ranunculus

litoralis, 438

Pallash, 958

Pallasii, 958

Ratibida

columnaris, 53

columnifera, 53

sulcata, 53

Ravenelia, 257

Rhamphocarya integrifoliolata, 183

Rhinanthus

minor

borealis, 787

Rhizophidium difficile, 50

Rhopal anthus, 902

Rhossalanthus mnioides, 902

Rhus, 915

angustifolium, 105

Copallinum, 103

Coriaria, 103

Filicina, 800

javanica, 103

potentillaefolia, 800

radicans, 103

Tetlatziam, 800

Toxicodendr., 103
Rhynchocarpus, 782

Rhynchosia difformis, 824

Rhynchospora filifolia, 874

glauca chinensis, 330 condensata, 783

lavarum, 330 spicaeformis, 759

lavarum, 330 spicaeformis, 759

p seudo-Sellowiana, 874

rugosa, 330

Sellowiana, 874

spicaeformis, 684, 759

spiciformis, 684

Rhytisma

astericolum, 286

solidum, 590

Rivinia

humilis, 337 canescens, 337

Robinia

Caragana, 942

Pseud-Acacia, 802

PseudoAcacia, 802

Rollandia

lanceolata, 73

grandifolia, 73

Rockii, 73

typica, 73

Rooseveltia, 384

Ropalon, 531

Rosa

acicularis

lacorum, 774

lacuum, 774

lapwaiensis, 106

Rosales, 58

Roschera, 205

Roscheria, 205

Rosineae, 406

Roxburghia, 682

Rubus

alceaefolius, 642

alceifolius, 642

Grimesii, 90

rosaefolius, 517

rosifolius, 517

Rudbeckia

columnaris, 53

columnifera, 53

purpure a

serotina, 317

serotina, 317 
Rufacer

carolini anum, 304

rubrum, 304

Rumex

Britannica, 674

Sabal

adiantinum, 301

Derringiana, 301

louisiana, 301

Sagenia

plataphylla, 280

platyphylla, 280

Sagittaria

calycina, 364

Hitchochii, 575

Saintpierrea, 554

Salacia, 620

Salix

alaxensis, 761

alexensis, 761

Hippophaefolia, 104

speciosa

Alaxensis, 761

Salsola

fruticosa, 950

Salvia

afr. lutea, 798

aurea, 798

Sanchezia, 865

Sanicula

canadensis

genuina, 631

typica, 631

Santolina

Chamae Cyparissus, 700

Chamae Cyparissus, 700

Chamaecyparissus, 700

chamaecyparissus, 700

Sarea

brassicaecola, 259

Sarmienta, 864

Sassafras

albida

glauca, 391

albidum, 391

molle, 391

officinale albidum, 391

officinalis, 391

Sassafras, 391

triloba, 391

Tzumu, 897

variifolium, 391

albidum, 391
Saurauia

roseotincta, 333

Saussurea

oxydonta, 767

Saxifraga occidentalis

wallowensis, 805

Saxo-fridericia, 446

Scae vola

Chamissoniana typica, 22

Schadjaret elharneb, 434

Schiedea

Gregoriana, 613

kealiae, 613

salicaria, 536

typica, 536

Schinus myricoides, 666

Schizanthus, 866

Schizophyllum, 168

Schlotheimia torquata, 297

Schmidia, 848

Schmidtia, 848

Schoenus

Cephalotes, 318

rugosus, 330

Schrebera, 367

Schinoides, 666

Scirpus

capitatus, 840

Cephalotes, 318

erecto-gracilis, 501

erectus, 501

lateralis, 501

lateriflorus, 501

macrostachyos, 522

maritimus

macrostachyus, 522

paludosus, 522

robustus, 522

swampianus, 795

uninodis, 501

Sclerotinia

bifrons, 518

confundens, 518

Whetzelii, 518

Sclerotium

bifrons, 518

Scolecotrichium

Asclepiadis, 388

Scoria, 301

Scrophularioides

arborea, 633 
Scutellaria

elliptica, 931

integrifolia, 931

nemorosa, 931

ovalifolia, 931

mollis, 931

pilosa

ovalifolia, 931

teucrifolia, 931

Scyphofilix, 270

trrigosa, 270

Sechium, 919

Secondatia difformis, 592

Secula viscidula, 680

Selagina, 334

Selaginurus, 334

Selinum c anadense, 410

Senecio

Alberti-Smithii, 566

Danielis, 662

Fortunatus, 567

Guadalupe, 867

littoralis, 438

mortuosus, 718

Szyszylowiczii, 578

Serrafalcus

Lloydianus, 740

Sesuvium

Portulac astrum, 56

Shorea

costata, 514

Sida

acuta, 457

Holtzii, 1

lepida, 905

lepidota, 905

Meyeniana, 904

genuina, 904

paniculata, 895

panniculata, 895

potentilloides, 412

pseudo-potentilloides, 412

Sidalcea, 50

secundiflora, 50

Siderocarpus, 400

Silagurium longifolia, 457

Silene

Antirrhina, 860

Silphium

terebinthinaceum

Lucy-Brauniae, 799
Sinclairia, 424

Sison

Ammi, 407

Smelowskia

Californica, 527

Smilax

Pseudo-China, 820

tamnifolia, 820

tamnoides, 820

Solanum

incompletum, 664

Nelsoni

thoma siaefolium, 727

sandwicense, 579

vestitum, 727

vulgare, 102

Woahense, 579

Solidago

ulmifolia

Palmeri, 847

Sorbaronia, 152

monstrosa, 152

Sorbus

americana, 887

micrantha, 887

microcarpa, 887

Spegazzinia, 655

Spermacoce

diodina, 609

Sphaeria, 281

coccinea, 625

decidua, 625

glomus, 649

picea, 241

Sphaerobotryotinia, 273

Spirotecoma, 427

Spondias

acida, 2

pleiogyna, 2

Solandri, 2

Spongia

oculata, 41

Spongocladia, 203

Spongodendron, 203

Stapfia, 729

Stellandria, 120

Stemodia, 172

Stenoloma, 880

Stephanospermum stewarti, 436

Sterigmatocy stis minor, 430

Stewartia montana, 708 
Stictocardia tiliaefolia, 18 tiliifolia, 18

Stipa, 657

Straussia Kaduana, 888

Stravadium, 610

Streptomyces albo-niger, 274

Struthiopteris pensylvanica, 217

Styphelia

Banksii, 760

Douglasii, 612

Grayana, 612

Tameiameiae, 785

Suaeda, 908

australis nova zelandica, 950

fruticosa, 950

vermiculata, 908

Succuta, 367

Sussuela

esculentum, 502

Suzygium, 379

Svertia, 393

Swertia, 393

Swietenia

Mahagoni, 949

Mahogani, 949

Symingtonia, 65

Syzy gium, 379

Tabernaemontana

Echites, 933

Tacca

maculata, 861

Taxodium

Washingtonium, 707

Tectaria

filix mas, 954

Telopea, 731

Tephrosia, 16

Terminalia mollis, 306

Tetracheilos Meisneri, 589

Tetranthera albida, 391

Teucrium cubense den sum, 171 depressum, 171 depressum, 171
Thelephora

amorpha, 210

candida, 513

candidissima, 513

laevis, 747

Thelypteris, 8

Dryopteris, 89

simulata, 606

spinulosa, 300

Thermopsis

pauciflora, 389

Thespesia, 878

Thryocephalon nemorale, 318

Thyrsigerae, 650

Tiarella cordifolia typica, 525

Tillaea, 556

Toluifera, 626

Tolypella, 853

Tolypiocladia, 205

Torreya, 326

californica, 326

Myristica, 326

Toxicodendron potentillifolium, 800

Trachelospermum difforme, 592

Trachyspora, 296

Trechispora, 296

Trematocarpus, 730 macrostachys, 730

Trematolobelia grandifolia, 323

macrostachys, 323, 730

grandifolia, 323

sandwicensis, 730

Trematosphaeria Morthieri, 833

Trematosphaeriopsis, 164

Trematosphaeris, 164

Trematostoma Morthieri, 833

Tremella botryoides, 263

Nostoc, 447

Tremellodendron candidum, 513

Trialatae, 54

Trichogloea Herveyi, 211

Tricholoma quercifolia, 206 subfulvidiscum, 206 
Trichomanes, 838

poly anthos, 264

strigo sum, 270

Trifolium

agrarium, 417

campestre, 417

dubium, 417

filiforme, 417

minus, 417

procumbens, 417

Trisetum

flavescens

pratense, 630

pratense, 630

Tritica, 807

Triticum, 807

elymogenes, 175

junceum $\times$ Elymus arenarius, 175

junceum $\times$ Hordeum arenarium, 175

pauciflorum, 953

strictum, 175

trachycaulum, 953

Tritordeum, 415

strictum, 175

Triumfetta, 870

Trochodendroxylon

Beckii, 573

Trymalium

ramosissimum, 724

Tsjeru-parua, 457

Tsuga

Jeffreyi, 182

Pattoniana Jeffreyi, 182

Tsugo-Piceo-Tsuga

Jeffreyi, 182

Tulasnella metallica, 458

Tumion, 326

californicum, 326

Ulmus

fulva, 868

rubra, 868

Umbelliferae, 541

Uredo

appendiculata, 226

hyperici, 571

Pamparum, 226

Polygoni, 359

rufa, 226

Violae, 165
Urera

Bequaertii, 896

glabra

mollis, 414

sandwicen sis mollis, 414

Urnigerae, 356

Uromyces

appendiculatus, 226

Hyperici, 571

triquetrus, 571

Urophyllum

Johannis Winkleri, 350

Urostachys

erubescens, 231

Rolandii-principes, 701

rolandi-principis, 701

sulcinervis

kanaiensis, 742

Urostigma

benjamineum

nud a, 709

nudum, 709

Uvularia

puberula, 32

pudica, 32

Vandenboschia, 838

Varengevillea

hispidissima, 188

Variegatae, 738

Verbena

litoralis, 438

Verbesina

alba, 618

prostrata, 618

Vernonia

Baldwini, 640 interior, 640

interior, 640

Baldwini, 640

Veronica

catenata, 884

comosa glaberrima, 884

connata glaberrima, 884

hybrida, 376

spuria, 376

Veronicena, 376

Haartmani, 376

Verrubotrytis, 272

Verrucaria, 392

Verrucariomyces, 392 
Verticilis

tuberculis, 275

Verticilliodochium

tubercularioides, 275

Verticillium

tubercularioides, 275

Viburnum

nudum

angustifolium, 91

Victoria

amazonica, 319

amazonum, 319

Cruziana, 319

regalis, 319

Regia, 319

regina, 319

reginae, 319

Vigna

Catjang, 639

cylindrica, 639

Vilfoidea, 862

Vinca

rosea, 644

Vincentia

an gustifolia, 632

Viola

Chamis soniana, 21 beta, 21

Kauaensis, 48

Kauai ensis, 48

Kauensis, 48

Kavaiensis, 48

Kawaiensis, 48

Viorna, 356

Vitex

trifolia

trifoliata, 500

trifoliolata, 500

Volvariopsis

Earleae, 595

Earlei, 595

Volvox

capensis, 437
Vredo

betae

Vredo Conuoluuli, 15

Vrydagzynea, 94

Weinmannia

borneensis, 634

Whitia, 782

Wikstroemia, 460

Wikströmia, 460

Wyethia

amplexicaulis, 14

Xamesike

supina, 746

Xylaria

Cornu Dorcas, 433

Xylosma, 626

Yucca

angustifolia, 418

glauca, 62, 418

Zanardinula

Kylinii, 227

lanceolata, 227

linearis, 227

Zanthoxylum

Kauaense, 45

Kauaiense, 45

Zerumbet, 146

Zignoella

Morthieri, 833

Zingiber, 146

Zinziber, 146

Zonaria, 229

varie gata, 779

Zschokkea, 944

Zschokkia, 944

Zygia, 400

Zygnema

insigne confusospora, 278

novae-caesareae, 416 







Ciz

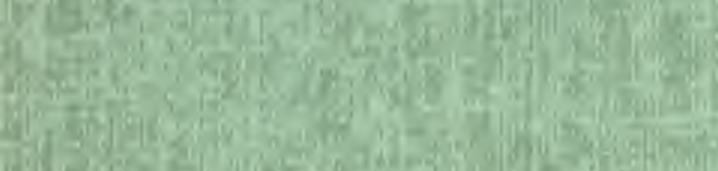

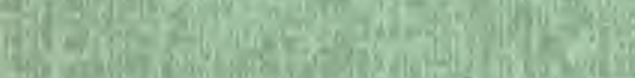

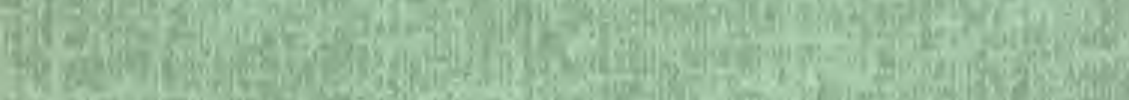
(10. 3. Q6y Fy H A 20.

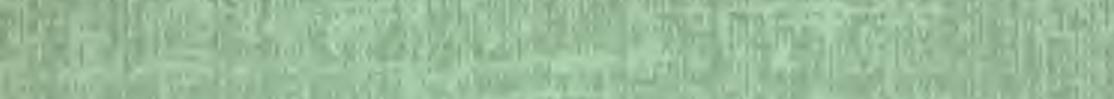
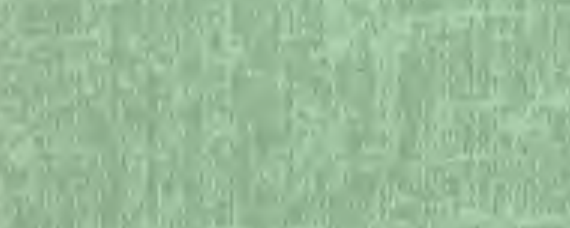

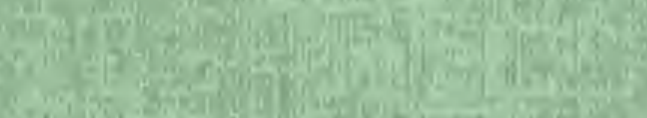
Q hamilingit? tons

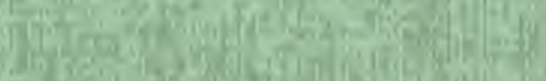
$\left(\begin{array}{ll}3 \\ 3\end{array}\right.$ T.5. vathentit Mos $2-x+1$ (1) $\sin 5$

120 $1,240+12$

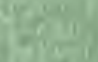
H.

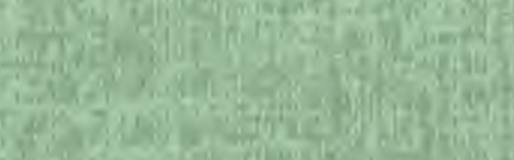

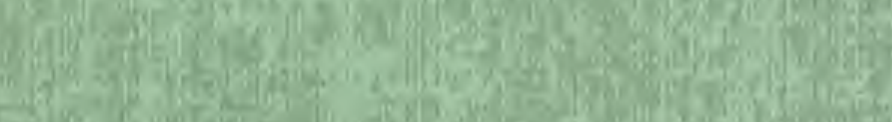

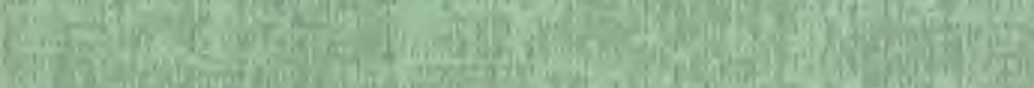

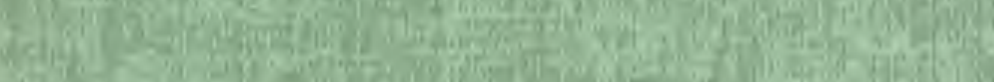

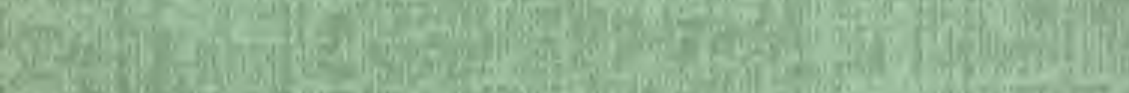

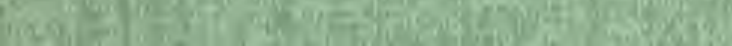


Fist 